\title{
SUPERPOSITION OF DIFFUSIONS WITH LINEAR GENERATOR AND ITS MULTIFRACTAL LIMIT PROCESS*
}

\author{
ENDRE IGLÓI ${ }^{1}$ AND GYÖRGY TERDIK ${ }^{1}$
}

\begin{abstract}
In this paper a new multifractal stochastic process called Limit of the Integrated Superposition of Diffusion processes with Linear differencial Generator (LISDLG) is presented which realistically characterizes the network traffic multifractality. Several properties of the LISDLG model are presented including long range dependence, cumulants, logarithm of the characteristic function, dilative stability, spectrum and bispectrum. The model captures higher-order statistics by the cumulants. The relevance and validation of the proposed model are demonstrated by real data of Internet traffic.
\end{abstract}

Mathematics Subject Classification. 62M10, 60J60, 60G10, 93E12.

Received May 14, 2002.

\section{Contents}

Introduction

1. Multifractality, self-similarity and dilative stability

2. Diffusion processes with a linear differential generator (DLG processes) 27

2.1. Characterization of the DLG process

2.2. Cumulants, characteristic functional and spectra of the DLG process

3. Superposition of DLG processes and its multifractal limit process

3.1. Superposition of random-parameter stationary DLG processes (SDLG process) 39

3.2. Multifractal limit processes (LISDLG and $\triangle$ LISDLG processes) 46

3.3. Spectra of the SDLG process and its offsprings $\quad 56$

3.4. Nonlinearity of the $\triangle$ ISDLG and $\triangle$ LISDLG processes $\quad 72$

3.5. Relation with [1], a similar result of Barndorff-Nielsen $\quad 73$

4. Application to computer network traffic data $\quad 76$

$\begin{array}{lr}\text { 4.1. The dilative stability and parameter estimation } & 76\end{array}$

4.2. Fitting of distributions and spectra $\quad 79$

5. Open problems and directions of future research $\quad 81$

$\begin{array}{lll}\text { Appendix A. Multivariate } \Gamma \text { distribution } & 84\end{array}$

$\begin{array}{ll}\text { References } & 87\end{array}$

\footnotetext{
Keywords and phrases: Fractals, long range dependence, self-similarity, stationarity, higher order statistics, bispectrum, network traffic, superposition, diffusion processes, CIR process, DLG process, square root process.

* This research is supported by the Hungarian National Science Foundation OTKA No. T 032658.

1 Institute of Mathematics and Informatics, University of Debrecen, 4010 Debrecen, PF 12, Hungary;

e-mail: terdik@cic.unideb.hu
}

(C) EDP Sciences, SMAI 2003 


\section{INTRODUCTION}

Computer network traffic has recently been the subject of various types of statistical studies including fractal analysis, and in particular, measuring and modeling Long-Range Dependence (LRD), investigating self-similarity [26] and showing multifractal properties [39]. The common agreement among several empirical findings about the general properties of traces is summarized in [31] as follows:

- many signals show significant LRD, but behavior inconsistent with strict self-similarity;

- for many signals, the scaling behavior of moments as the signal is aggregated is a nontrivial function of the moment order;

- many signals have increments that are inherently positive, skewed and hence non-Gaussian.

There are some additional properties motivated by our experimental study of ATM traces, see [41], providing strong evidence of the presence of $\Gamma$ distribution and real-valued bispectrum. Therefore there are two additional requirements:

- the marginal distribution of signals of ATM traces is close to $\Gamma$ distribution;

- signals of ATM traces have a real-valued bispectrum.

Having these properties in mind we will study in the present paper a certain nonlinear diffusion process, superposition of such processes with random coefficients, the limit of the centralized integral processes of the superposition processes and its increment process. We will consider a possible application too. The main objective is to find a multifractal model which has an analytically and statistically tractable higher order cumulant structure.

The paper starts with the introduction. In Section 1 we introduce the notions of multifractality, selfsimilarity and dilative stability. In Section 2 the basic process of superposition is considered. We call it Diffusion with Linear differential Generator (DLG). We summarize the results obtained by Watanabe and his coauthors concerning the connection between the DLG process and infinitely decomposable diffusion processes and continuous-state branching processes. It is also pointed out that the finite-dimensional distribution of the DLG process is multivariate $\Gamma$. We are concerned with stationary observations, therefore the stationary DLG process will be studied in greater detail. The exact cumulants, characteristic functional and spectra of the stationary DLG process will be given.

In Section 3 we will consider a triangular array of random coefficient DLG processes. It will be shown that the row sums converge to a limit process called Superposition of DLG processes (SDLG process). As for the properties of the SDLG process we will prove that the logarithm of the characteristic functional of the SDLG process is the mixture of the logarithm of the characteristic functionals of the component DLG processes. The SDLG process also has a $\Gamma$ finite-dimensional distribution. It is stationary, it has a real-valued positive bispectrum and it is LRD. The main result of the paper is the existence of the Limit of Integrated SDLG processes, referred to as the LISDLG process. It is shown that both the LISDLG process and its increment process, labeled as $\triangle$ LISDLG, are multifractals. The covariance structure of the LISDLG process is the same as that of the Fractional Brownian Motion (FBM). Consequently, the spectrum of the $\Delta$ LISDLG process is the same as that of the discrete time Fractional Gaussian Noise (FGN), $\Delta B^{(H)}(k)=B^{(H)}(k+1)-B^{(H)}(k)$, $k \in \mathbb{Z}$. The bispectrum of the $\Delta$ LISDLG process is also given, and it has the extraordinary property that it is real-valued. If the bispectrum of a linearly regular long-range dependent discrete time process is real (non-zero), then the process must be nonlinear. Hence, the $\triangle$ LISDLG process is nonlinear. At the end of Section 3 we compare our results with those of [1]. In this context it is pointed out that the OrnsteinUhlenbeck (OU) type models considered in [1] cannot fulfil our requirements for the bispectrum to be real. The logarithm of the $m$-th order cumulants of the LISDLG process scales linearly with the logarithm of time. A similar scaling behavior also holds for the $\Delta$ LISDLG process. In particular (1.9) below means that the logarithm of the $m$-th order cumulants of the series averaged at level $n$ depends linearly on $\log n$ with coefficient $2(H-1)$ being independent of $m$, where $H$ is the Hurst parameter. This fact is more special than the multifractal property, nevertheless it holds under more general circumstances than our case, e.g. for the OU type models of [1], see Section 3.5. Moreover, the LISDLG process has an interesting property, which the authors call dilative stability, which implies the above mentioned scaling behavior (1.9).

In Section 4 we will apply our $\triangle$ LISDLG model to real data. The time series of ATM traces measured in SUNET fits our model very well. The feasibility of carrying out parameter estimation utilizing the dilative 
stability is also discussed to some extent. In Section 5 some open problems and possible research directions are suggested.

An Appendix giving some basic facts of multivariable $\Gamma$ distribution closes the paper.

\section{Multifractality, SELF-Similarity And Dilative StabiLity}

A wide variety of physical systems including data network traffic exhibit fractal properties. We are interested in fractal data, i.e. data "look the same across a wide range of scales", at least in certain respect. The notion of fractal, in the sense that it is similar on all scales, is translated into the stochastic analysis by the definition of Self-Similar process with Stationary Increments, see [23]. We will denote it by SSSI. The stochastic process $Y(t)$ is called $H$-SSsi, if it has stationary increments and, for all $a>0$ real numbers,

$$
Y(a t) \stackrel{d}{=} a^{H} Y(t)
$$

where $\stackrel{d}{=}$ means the equality of finite-dimensional distributions. The parameter $H$ is referred to as the Hurst parameter or Hurst exponent. An example is the FBM $B^{(H)}(t)$, where parameter $H \in(0,1)$, see $[2,29,33]$. Let $m \in \mathbb{N}$ and suppose that the $m$-th order cumulant, $\operatorname{cum}_{m}(Y(t))$, exists. Now it follows from selfsimilarity that

$$
\operatorname{cum}_{m}(Y(t))=t^{m H} \operatorname{cum}_{m}(Y(1))
$$

i.e. the logarithm of $\operatorname{cum}_{m}(Y(t))$ scales linearly with $\log (t)$ and the coefficient is the homogeneous linear function (of $m$ ) $m H$. Note that [39] considers absolute moments instead of cumulants, i.e.

$$
\mathrm{E}|Y(t)|^{m}=t^{m H} \mathrm{E}|Y(1)|^{m}
$$

instead of (1.1). For $H$-SSSI processes both (1.1) and (1.2) hold.

There are different generalizations of the self-similar processes to multifractal processes. One concerns the almost sure fractal property of continuous paths according to the deterministic theory of fractals, see [18]. The other one is based on the statistical inference of the process. We follow this latter direction. A generalization of the scaling (1.2) is

$$
\log \mathrm{E}|Y(t)|^{m}=m H(m) \log (t)+c(m)
$$

where the dependence of $H$ on $m$ points on the multifractal behaviour of the process. The papers [4] and [39] define the notion of multifractal essentially by (1.3). We prefer cumulants rather than absolute moments for describing the multifractal property because the scaling properties should not change with additive constants and with summing up of independent copies of a process.

Definition 1. A stochastic process $Y(t)$ is called multifractal if, for each $m \in \mathbb{N}$ the cumulant $\operatorname{cum}_{m}(Y(t))$ exists and the logarithm of $\left|\operatorname{cum}_{m}(Y(t))\right|$ scales linearly with $\log (t)$, and the coefficient is $m H(m)$, i.e.

$$
\log \left(\left|\operatorname{cum}_{m}(Y(t))\right|\right)=m H(m) \log (t)+c(m) .
$$

Note that in the above definition constant $c(m)$ is possibly $-\infty$, if the corresponding cumulant is zero; in this case (1.4) holds with arbitrary $H(m)$. Note also that $H(m)=H$ for $H$-sssi processes.

The $H$-Sssi property of $Y(t)$ implies that the increment process

$$
X(k)=Y(k+1)-Y(k), \quad k \in \mathbb{Z},
$$

which is stationary, is characterized in terms of the behavior of the aggregated processes arising by averaging $X(k)$ over nonoverlapping blocks of size $n \in \mathbb{N}$, i.e.

$$
X^{(n)}(k)=\frac{1}{n} \sum_{j=0}^{n-1} X(k n-j), \quad k \in \mathbb{Z}
$$


have similar finite-dimensional distributions, specifically,

$$
\forall n \in \mathbb{N}: X^{(n)}(k) \stackrel{d}{=} n^{H-1} X(k) .
$$

The stationary process $X(k)$ fulfilling (1.7) is called Stationary Self-Similar with Hurst exponent $H$, i.e. an $H$-sss process. A typical example is the FGN process $X(k) \stackrel{\circ}{=} \Delta B^{(H)}(k)=B^{(H)}(k+1)-B^{(H)}(k), k \in \mathbb{Z}$, which is a unique Gaussian $H$-sss process, see $[22,37]$. The $m$-th order cumulants of the aggregated $H$-sss processes also scale as

$$
\operatorname{cum}_{m}\left(X^{(n)}(k)\right)=n^{m(H-1)} \operatorname{cum}_{m}(X(k)) .
$$

Now if a stationary process $X(k)$ fulfils equation (1.8) for each $n \in \mathbb{N}$ and $m \in \mathbb{N}$, then the logarithm of the modulus of $\operatorname{cum}_{m}\left(X^{(n)}(k)\right)$ scales linearly with $\log (n)$ with coefficients $m(H-1)$ depending on $m$ homogeneous linearly. Therefore such processes will be called unifractal processes because the Hurst exponent $H$ is universal for all $n \in \mathbb{N}$ and $m \in \mathbb{N}$. The assumption that a process $Y(t)$ is $H$-Sssi is of course stronger than the assumption that the increment process is unifractal. One can go even further in weakening the assumption.

Definition 2. A stationary process $X(k), k \in \mathbb{Z}$, is multifractal if

$$
\log \left(\left|\operatorname{cum}_{m}\left(X^{(n)}(k)\right)\right|\right)=m(H(m)-1) \log (n)+c(m),
$$

for all $m \in \mathbb{N}$, i.e. allowing the exponent $H$ to vary with the order $m$.

Similarly to Definition 1 , constants $c(m)$ may be $-\infty$.

A particular class of multifractal processes is the set of $p^{\frac{1}{2(H-1)}}$-dilative stable processes which have cumulants of all orders.

Definition 3. We say that a process $Y(t)$ is dilative stable with scaling function $c: \mathbb{R}_{+} \rightarrow \mathbb{R}_{+}$(or $c$ dilative stable, for short), if for every $p>0, k \in \mathbb{N}$, and $t_{1}, \ldots, t_{k} \geqslant 0$, the characteristic function $\varphi$ of the $k$-dimensional distribution satisfies

$$
\left(\varphi_{\left(Y\left(t_{1}\right), \ldots, Y\left(t_{k}\right)\right)}\left(u_{1}, \ldots, u_{k}\right)\right)^{p}=\varphi_{\frac{1}{c(p)}\left(Y\left(c(p) t_{1}\right), \ldots, Y\left(c(p) t_{k}\right)\right)}\left(u_{1}, \ldots, u_{k}\right),
$$

for all $u_{1}, \ldots, u_{k} \in \mathbb{R}$.

Note that the attribute "dilative" alludes to the dilatation $\left(t_{1}, \ldots, t_{k}\right) \mapsto c(p)\left(t_{1}, \ldots, t_{k}\right)$ in the time domain. If cumulants of all orders of $Y(t)$ exist, then the $c(p)=p^{\frac{1}{2(H-1)}}$-dilative stability implies that

$$
p \operatorname{cum}_{m}(Y(t))=p^{\frac{m}{2(1-H)}} \operatorname{cum}_{m}\left(Y\left(p^{\frac{1}{2(H-1)}} t\right)\right),
$$

and taking $p:=t^{2(H-1)}$ and $t:=1$, we have

$$
\log \left(\left|\operatorname{cum}_{m}(Y(t))\right|\right)=(2(H-1)+m) \log t+\log \left(\left|\operatorname{cum}_{m}(Y(1))\right|\right),
$$

i.e.

$$
\log \left(\left|\operatorname{cum}_{m}\left(\frac{Y(t)}{t}\right)\right|\right)=2(H-1) \log t+c(m) .
$$

Similarly, it holds for the aggregated process (1.6) of the increment process (1.5), that

$$
\log \left|\operatorname{cum}_{m}\left(X^{(n)}(k)\right)\right|=2(H-1) \log (n)+c(m) .
$$

What is interesting is that the coefficients of $\log t$ and $\log n$ do not depend on $m$. However, this must not be confused with the unifractal property; it is an outcome of the dilative stability. 
The increments of an $H$-SSSI process $Y(t)$ have another particular second-order property called LongRange Dependence (LRD). The definition of LRD for a stationary process $X(k), k \in \mathbb{Z}$, is that the autocovariance series $r(k) \stackrel{\circ}{=} \operatorname{cov}(X(i), X(i+k))=\operatorname{cum}_{2}(X(i), X(i+k)), k \in \mathbb{Z}$, behaves asymptotically as

$$
r(k) \underset{k \rightarrow \infty}{\simeq} L(k) k^{2(H-1)},
$$

where $L(x)$ is a slowly varying function at infinity, and $1 / 2<H<1$. Note that the slowly varying function has not a significant role. A stationary process is asymptotically second-order self-similar, see [8], if the relationship

$$
\forall n \in \mathbb{N}: r^{(n)}(k)=\operatorname{cum}\left(X^{(n)}(i), X^{(n)}(i+k)\right)=n^{2(H-1)} r(k)
$$

holds asymptotically as $n \rightarrow \infty$ and $k \rightarrow \infty$, or more precisely, the series of the autocorrelation functions $\rho^{(n)}(k)=r^{(n)}(k) / r^{(n)}(0)$ converges, as $n \rightarrow \infty$, to an autocorrelation series $\rho^{(\infty)}(k), k \in \mathbb{Z}$, and $\rho^{(\infty)}$ and $\rho^{(1)}$ are equivalent at infinity, i.e. $\rho^{(\infty)}(k)$ and $\rho^{(1)}(k)$ converge to zero in the same order as $k \rightarrow \infty$, i.e.

$$
\rho^{(\infty)}(k) \underset{k \rightarrow \infty}{\simeq} \text { const. } \rho^{(1)}(k) .
$$

This type of self-similarity is the weakest and it is the one which is implied by LRD. For an example see the $\Gamma M O U$ process in [16]. The main subject of this paper, the $\Delta$ LISDLG process is not self-similar, but as it is LRD, it is asymptotically second-order self-similar.

\section{Diffusion processes With a LinEAR DifFERENTIAL GENERATOR (DLG PROCESSES)}

\subsection{Characterization of the DLG process}

Let us consider the diffusion-type stochastic differential equation

$$
\mathrm{d} R(t)=(\mu+2 \alpha R(t)) \mathrm{d} t+2 \sigma \sqrt{R(t)+} \mathrm{d} B(t)
$$

where $R(t)_{+} \doteq \max (R(t), 0)$, moreover $\mu>0, \alpha \in \mathbb{R}, \sigma>0$ are the parameters, and $B(t)$ is a Brownian motion. The equation (2.1) has a unique strong solution $R(t)$, see the paper [43] or Theorem IV.3.2 in [17] about the existence and uniqueness of the solution. It is not hard to prove that solution $R(t)$ is conservative, because $P(R(t) \geqslant 0 \mid R(0) \geqslant 0)=1$ for all $t \geqslant 0$, see Example IV.8.2 in [17]. Assuming that $R(0) \geqslant 0$ one could omit the notation of the nonnegative part in (2.1), replacing $R(t)_{+}$by $R(t)$, i.e.

$$
\mathrm{d} R(t)=(\mu+2 \alpha R(t)) \mathrm{d} t+2 \sigma \sqrt{R(t)} \mathrm{d} B(t)
$$

Put

$$
\delta \stackrel{\rho}{=} \frac{\mu}{\sigma^{2}}
$$

The solution $R(t)$ of the stochastic differential equation (2.2) will be treated below under the name $\delta$ dimensional Diffusion process with a Linear differential Generator (DLG process), because its differential generator $A$ is given by

$$
A=(\mu+2 \alpha x) \frac{\partial}{\partial x}+2 \sigma^{2} x \frac{\partial^{2}}{\partial x^{2}},
$$

and both coefficients of $A$ are linear, see [11]. Note here that the DLG process has recently been used successfully for modeling interest rates, because it is one of the simplest models avoiding negative values. There is a lack of agreement on terminology: after the authors of [9], for example, reference [36] would 
label this as CIR process and in the special case $\alpha=0$ (we will have mostly $\alpha<0$ ) it would be called the squared Bessel process. Because of the form (2.1) it is called square-root diffusion, too. In the mathematical literature this process has not been denominated although it was mentioned by Kolmogorov in 1931, see [21] and was studied intensively by $[12,19]$ and by Watanabe in the seventies, see below.

Albeit at first sight the DLG process seems to be an overly special process, in fact the set of DLG processes is a parametric class of substantially general processes.

Proposition 1. If $\alpha<0$, and $\delta \in \mathbb{N}$, then the DLG process is just the squared radial part of a particular $\delta$-dimensional OU process.

Proof (by Chap. 31 of [36]). Let us take the OU processes $X_{i}(t), i=1, \ldots, \delta$, solutions of the Langevin equations

$$
\mathrm{d} X_{i}(t)=\alpha X_{i}(t) \mathrm{d} t+\sigma \mathrm{d} B_{i}(t)
$$

where $B_{i}(t), i=1, \ldots, \delta$, are independent Brownian motions. Let

$$
R(t) \stackrel{\circ}{=} \sum_{i=1}^{\delta} X_{i}(t)^{2}
$$

Then Itô's formula implies

$$
\mathrm{d} R(t)=\left(2 \alpha R(t)+\delta \sigma^{2}\right) \mathrm{d} t+2 \sigma \sqrt{R(t)} \sum_{i=1}^{\delta} \frac{X_{i}(t)}{\sqrt{R(t)}} \mathrm{d} B_{i}(t)
$$

Since

$$
B(t) \triangleq \sum_{i=1}^{\delta} \int_{0}^{t} \frac{X_{i}(s)}{\sqrt{R(s)}} \mathrm{d} B_{i}(s)
$$

is a continuous square-integrable local martingale (with respect to the product $\sigma$-field) with quadratic variation $t$, it follows by Lévy's theorem (see, e.g. Th. II.6.1 in [17]) that $B(t)$ is a Brownian motion. Thus, $R(t)$ fulfils $(2.2)$ with $\mu=\delta \sigma^{2}$.

Let $\alpha \in \mathbb{R}$ and $\sigma>0$ be arbitrarily fixed parameters.

\section{Notation 1.}

$$
R_{x}^{(\delta)}(t) \stackrel{\circ}{=} \text { solution of }(2.2) \text { with } \mu=\delta \sigma^{2} \text { starting from the initial value } x
$$

i.e. $R_{x}^{(\delta)}(0)=x$, and

$$
P_{x}^{(\delta)} \stackrel{\circ}{=} \text { distribution of } R_{x}^{(\delta)} \text { on } \mathcal{C}[0, \infty)
$$

for $x>0$ and $\delta>0$. Sometimes, when it is not important to feature the superscript $(\delta)$, we will omit it.

\section{Proposition 2.}

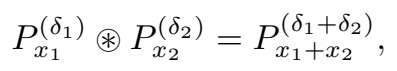

for arbitrary $\delta_{1}, \delta_{2}>0$ and $x_{1}, x_{2}>0$, where $\circledast$ denotes the convolution.

Proof. Let

$$
\mathrm{d} R_{x_{i}}^{\left(\delta_{i}\right)}(t)=\left(\delta_{i} \sigma^{2}+2 \alpha R_{x_{i}}^{\left(\delta_{i}\right)}(t)\right) \mathrm{d} t+2 \sigma \sqrt{R_{x_{i}}^{\left(\delta_{i}\right)}(t)} \mathrm{d} B_{i}(t),
$$


$i=1,2$, be the defining equations of the two DLG processes, where $B_{1}(t)$ and $B_{2}(t)$ are independent Brownian motions. Summing the two equations up, we have

$$
\begin{aligned}
\mathrm{d}\left(R_{x_{1}}^{\left(\delta_{1}\right)}(t)+R_{x_{2}}^{\left(\delta_{2}\right)}(t)\right)= & \left(\left(\delta_{1}+\delta_{2}\right) \sigma^{2}+2 \alpha\left(R_{x_{1}}^{\left(\delta_{1}\right)}(t)+R_{x_{2}}^{\left(\delta_{2}\right)}(t)\right)\right) \mathrm{d} t+2 \sigma \sqrt{R_{x_{1}}^{\left(\delta_{1}\right)}(t)+R_{x_{2}}^{\left(\delta_{2}\right)}(t)} \\
& \times\left(\frac{\sqrt{R_{x_{1}}^{\left(\delta_{1}\right)}(t)}}{\sqrt{R_{x_{1}}^{\left(\delta_{1}\right)}(t)+R_{x_{2}}^{\left(\delta_{2}\right)}(t)}} \mathrm{d} B_{1}(t)+\frac{\sqrt{R_{x_{2}}^{\left(\delta_{2}\right)}(t)}}{\sqrt{R_{x_{1}}^{\left(\delta_{1}\right)}(t)+R_{x_{2}}^{\left(\delta_{2}\right)}(t)}} \mathrm{d} B_{2}(t)\right) .
\end{aligned}
$$

Define

$$
B(t) \doteq \int_{0}^{t} \frac{\sqrt{R_{x_{1}}^{\left(\delta_{1}\right)}(s)}}{\sqrt{R_{x_{1}}^{\left(\delta_{1}\right)}(s)+R_{x_{2}}^{\left(\delta_{2}\right)}(s)}} \mathrm{d} B_{1}(s)+\int_{0}^{t} \frac{\sqrt{R_{x_{2}}^{\left(\delta_{2}\right)}(s)}}{\sqrt{R_{x_{1}}^{\left(\delta_{1}\right)}(s)+R_{x_{2}}^{\left(\delta_{2}\right)}(s)}} \mathrm{d} B_{2}(s) .
$$

Then one can use the same argument as at the end of the proof of Proposition 1 to get that $B(t)$ is a Brownian motion. Finally, let

$$
R_{x_{1}+x_{2}}^{\left(\delta_{1}+\delta_{2}\right)}(t) \doteq R_{x_{1}}^{\left(\delta_{1}\right)}(t)+R_{x_{2}}^{\left(\delta_{2}\right)}(t)
$$

Then we have from (2.6) and (2.7) that $R_{x_{1}+x_{2}}^{\left(\delta_{1}+\delta_{2}\right)}(t)$ is really a DLG process with dimension $\delta_{1}+\delta_{2}$ and starting from $x_{1}+x_{2}$. Since processes $R_{x_{1}}^{\left(\delta_{1}\right)}(t)$ and $R_{x_{2}}^{\left(\delta_{2}\right)}(t)$ are independent, we obtain $(2.5)$.

Notation 2. Let $\mathbf{P}^{(\delta)}$ be the equivalence class of distributions of $\delta$-dimensional DLG processes, i.e.

$$
\left.\mathbf{P}^{(\delta)} \stackrel{\ominus}{=} P_{x}^{(\delta)}: x>0\right\}
$$

for $\delta>0$. It is natural to call $\mathbf{P}^{(\delta)}$ the distribution class of $R_{x}^{(\delta)}$. Also define the parametric set

$$
\mathcal{P} \doteq\left\{\mathbf{P}^{(\delta)}: \delta>0\right\}
$$

Obviously, $\mathcal{P}$ is a classification of the set $\left\{P_{x}^{(\delta)}: \delta>0, x>0\right\}$ and it is compatible with respect to the convolution operation, so one can naturally extend the convolution to $\mathcal{P}$ via class representatives, i.e. with the aid of (2.5), as follows:

Definition 4. Let $\delta_{1}, \delta_{2}>0$. Then

$$
\mathbf{P}^{\left(\delta_{1}\right)} \circledast \mathbf{P}^{\left(\delta_{2}\right)} \stackrel{\circ}{=} \mathbf{P}^{\left(\delta_{1}+\delta_{2}\right)}
$$

Writing formula (2.5) in the language of Laplace transforms of finite dimensional distributions, and taking particular initial values, we have

$$
\mathcal{L}_{c \delta_{1}}^{\left(\delta_{1}\right)}(\underline{u}) \mathcal{L}_{c \delta_{2}}^{\left(\delta_{2}\right)}(\underline{u})=\mathcal{L}_{c\left(\delta_{1}+\delta_{2}\right)}^{\left(\delta_{1}+\delta_{2}\right)}(\underline{u})
$$

for every $c, \delta_{1}, \delta_{2}>0$, where $\mathcal{L}_{x}^{(\delta)}(\underline{u})$ denotes the Laplace transform at point $\underline{u} \in \mathbb{R}_{+}^{k}$ of the $k$-dimensional distribution of $\left(R_{x}^{(\delta)}\left(t_{1}\right), \ldots, R_{x}^{(\delta)}\left(t_{k}\right)\right)$, and $k \in \mathbb{N}$ and $t_{1}, \ldots, t_{k} \in \mathbb{R}_{+}$are arbitrary but fixed. Because Cauchy's multiplicative functional equation has a unique solution in the set of measurable functions, the solution of (2.9) is

$$
\mathcal{L}_{c \delta}^{(\delta)}(\underline{u})=\left(\mathcal{L}_{c}^{(1)}(\underline{u})\right)^{\delta},
$$


$\delta>0$. Considering (2.10) for fixed $c$ and $\delta$ and for every $k \in \mathbb{N}$ and every $\underline{u} \in \mathbb{R}_{+}^{k}$, we have

$$
P_{c \delta}^{(\delta)}=\left(P_{c}^{(1)}\right)^{\circledast \delta}
$$

where the convolution power is defined as the inverse Laplace transform of the right hand side of (2.10). This notion of convolution power is, clearly a generalization of the one with positive integer exponent. One could naturally extend it to $\mathcal{P}$ via class representatives, however, the following definition is sufficient for our purposes.

Definition 5. Let $c, \delta>0$.

$$
\left(\mathbf{P}^{(1)}\right)^{\circledast \delta} \doteq \text { the distribution class of }\left(P_{c}^{(1)}\right)^{\circledast \delta} \text {. }
$$

Obviously, this definition is right, because by (2.11), the class is independent of $c$. Now we formulate the statement (2.11) in a concise form as follows:

\section{Proposition 3.}

$$
\mathbf{P}^{(\delta)}=\left(\mathbf{P}^{(1)}\right)^{\circledast \delta}
$$

holds for every $\delta \in \mathbb{R}_{+}$.

As mentioned above, the set of DLG processes is a parametric class of substantially general processes. To explain it we introduce some notions.

Definition 6 (see [34]). A stochastic process is infinitely decomposable, if for every $n \in \mathbb{N}$ its distribution class $\mathbf{P}$ (i.e. the set of its distributions for all possible initial values) can be represented in the convolution power form

$$
\mathbf{P}=\left(\mathbf{P}_{n}\right)^{\circledast n},
$$

with some proper distribution classes $\mathbf{P}_{n}$.

Remark 1. Clearly (2.8) implies that the DLG process is infinitely decomposable.

Definition 7. A process $X(t)$ is a Continuous-state Branching process with Immigration (CBI process) with a.s. continuous trajectories, if $X(t)$ is a diffusion process with nonnegative trajectories, and there exist functions $f(t, u)>0$ and $g(t, u)>0, t, u>0$, such that the conditional Laplace transform of $X(t)$ has the form

$$
\mathrm{E}\left(\mathrm{e}^{-u X(t)} \mid X(0)=x\right)=\mathrm{e}^{-f(t, u) x} g(t, u)
$$

for all $x>0$.

We now list without proofs some statements from Section 1 of [34], which indicate the ponderosity of the DLG process.

Theorem 1 (see [34]). The following statements are equivalent:

1) $R(t)$ is a DLG process;

2) $R(t)$ is an infinitely decomposable diffusion process with nonnegative trajectories;

3) $R(t)$ is a $C B I$ process with a.s. continuous trajectories.

Theorem 1 is the elegant, and the following Theorem 2 is the exact version. 
Theorem 2 (see [34]). The following statements are equivalent:

1) $\left\{\mathbf{P}^{(\delta)}: \delta>0\right\}$ is a parametric set of the distribution classes of DLG processes with the same parameters $\alpha \in R$ and $\sigma>0$;

2) $\left\{\mathbf{P}^{(\delta)}: \delta>0\right\}$ is a parametric set of the distribution classes of diffusion processes with nonnegative trajectories, and it is a one-parameter convolution semigroup, i.e. (2.8) holds for all $\delta_{1}, \delta_{1}>0$;

3) $\left\{\mathbf{P}^{(\delta)}: \delta>0\right\}$ is a parametric set of the distribution classes of a.s. continuous CBI processes and (2.12) holds for all $\delta>0$.

Observe that (2.12) also follows from Theorem 2 .

We now examine the finite-dimensional distributions of the DLG process. Using the following result of Section 1 in [34], one can directly calculate the conditional characteristic function.

Lemma 1 (see [34]). Let $X(t)$ be a CBI process with a.s. continuous trajectories, and let the differential generator, corresponding to it by Theorem 1 , be of the form

$$
(\mu+2 \alpha x) \frac{\partial}{\partial x}+2 \sigma^{2} x \frac{\partial^{2}}{\partial x^{2}}
$$

Then functions $f(t, u)$ and $g(t, u)$ in $(2.13)$ are such that $f(t, u)$ is the solution of the differential equation

$$
\begin{aligned}
\frac{\partial}{\partial t} f(t, u) & =-2 \sigma^{2} f^{2}(t, u)+2 \alpha f(t, u) \\
f(0, u) & =u
\end{aligned}
$$

and

$$
g(t, u)=\exp \left(-\mu \int_{0}^{t} f(s, u) \mathrm{d} s\right) .
$$

Proposition 4. The conditional characteristic function of the instantaneous value of the DLG process, given its initial value, is

$$
\begin{aligned}
\varphi_{R^{(\delta)}(t)}\left(u \mid R^{(\delta)}(0)=x\right) & \stackrel{\circ}{=}\left(\mathrm{e}^{i u R^{(\delta)}(t)} \mid R^{(\delta)}(0)=x\right) \\
& =\exp \left(\frac{i u x \mathrm{e}^{2 \alpha t}}{1-i u \frac{\sigma^{2}}{-\alpha}\left(1-\mathrm{e}^{2 \alpha t}\right)}\right)\left(1-i u \frac{\sigma^{2}}{-\alpha}\left(1-\mathrm{e}^{2 \alpha t}\right)\right)^{-\frac{\delta}{2}},
\end{aligned}
$$

$u \in \mathbb{R}$.

Proof. By Theorem 2 it is enough to compute the conditional characteristic function of a CBI process with a.s. continuous trajectories and with property $(2.12)$. Let $\widetilde{R}^{(\delta)}(t)$ be such a process. Then the conditional Laplace transform of $\widetilde{R}^{(\delta)}(t)$ is of the form

$$
\begin{aligned}
& \psi_{\widetilde{R}^{(\delta)}(t)}\left(u \mid \widetilde{R}^{(\delta)}(0)=x\right) \doteq \mathrm{E}\left(\mathrm{e}^{-u \widetilde{R}^{(\delta)}(t)} \mid \widetilde{R}^{(\delta)}(0)=x\right) \\
& =\mathrm{e}^{-f(t, u) x} g(t, u),
\end{aligned}
$$

and by Lemma $1 f(t, u)$ and $g(t, u)$ are given by (2.14) and (2.15). The solution of (2.14) can be easily found by the replacement $h=1 / f$ to be

$$
f(t, u)=\frac{u \mathrm{e}^{2 \alpha t}}{1-u \frac{\sigma^{2}}{\alpha}\left(1-\mathrm{e}^{2 \alpha t}\right)} .
$$


Hence, by (2.15),

$$
g(t, u)=\left(1-u \frac{\sigma^{2}}{\alpha}\left(1-\mathrm{e}^{2 \alpha t}\right)\right)^{-\frac{\delta}{2}}
$$

Thus, by (2.17) the Laplace transform

$$
\psi_{\widetilde{R}^{(\delta)}(t)}\left(u \mid \widetilde{R}^{(\delta)}(0)=x\right)=\exp \left(\frac{-u x \mathrm{e}^{2 \alpha t}}{1+u \frac{\sigma^{2}}{-\alpha}\left(1-\mathrm{e}^{2 \alpha t}\right)}\right)\left(1+u \frac{\sigma^{2}}{-\alpha}\left(1-\mathrm{e}^{2 \alpha t}\right)\right)^{-\frac{\delta}{2}}
$$

whereby we get the characteristic function in the statement.

Formula (2.16) can be obtained also in a more direct probabilistic way, see [17], IV, 8, formula (8.10) and below.

The transition density of the DLG process can be derived following [19], see also [12]. We quote only the resulting formula:

Proposition 5. If $\alpha<0$, then the transition density $p_{x}(t, y) \doteq p_{R^{(\delta)}(t) \mid R^{(\delta)}(0)}(y \mid x)$ of the DLG process is

$$
p_{x}(t, y)=\frac{-\alpha}{\sigma^{2}} \cdot \frac{1}{1-\mathrm{e}^{2 \alpha t}} \exp \left(\frac{\alpha}{\sigma^{2}} \cdot \frac{y+x \mathrm{e}^{2 \alpha t}}{1-\mathrm{e}^{2 \alpha t}}\right)\left(\frac{x}{y} \mathrm{e}^{2 \alpha t}\right)^{-\beta / 2} J_{\beta}\left(\frac{2|\alpha|}{\sigma^{2}} \cdot \frac{\sqrt{x y \mathrm{e}^{2 \alpha t}}}{1-\mathrm{e}^{2 \alpha t}}\right)
$$

where $\beta \doteq \mu /\left(2 \sigma^{2}\right)-1$ and $J_{\beta}$ denotes the modified Bessel function of the first kind of order $\beta$.

To guarantee the stationarity of the processes under consideration, from now on we will restrict the set of values of the parameter $\alpha$ to negative values, i.e. the condition $\alpha<0$ will hold in the rest of the paper.

Taking the limit as $t \rightarrow \infty$ in (2.16), because $\alpha<0$, we have the characteristic function of the stationary distribution

$$
\varphi_{R^{(\delta)}(t)}(u) \stackrel{\circ}{=} \mathrm{e}^{i u R^{(\delta)}(t)}=\lim _{t \rightarrow \infty} \varphi_{R^{(\delta)}(t)}\left(u \mid R^{(\delta)}(0)=x\right)=\left(1-i u \frac{\sigma^{2}}{-\alpha}\right)^{-\frac{\delta}{2}}
$$

i.e. the characteristic function of the $\Gamma\left(\delta / 2,-\sigma^{2} / \alpha\right)$ distribution. The same stationary distribution could be obtained by taking the limit as $t \rightarrow \infty$ in (2.18). Thus we can state the following:

Proposition 6. The one-dimensional stationary distribution of the $\delta$-dimensional DLG process $R(t)$ defined by $(2.2)$, where $\alpha<0$, is $\Gamma$, i.e.

$$
R(t) \sim \Gamma\left(\frac{\delta}{2}, \frac{\sigma^{2}}{-\alpha}\right)
$$

In the rest of the paper we will deal with stationary DLG processes, i.e. we will assume that the stationary distribution is attained. In other words, we will suppose that the process starts from its stationary onedimensional distribution.

Let us examine the $m$-dimensional distribution of the stationary DLG process.

Proposition 7. Let $\underline{t} \stackrel{\circ}{=}\left(t_{1}, \ldots, t_{m}\right)$, and $t_{1}<\ldots<t_{m}$. Then the $m$-dimensional stationary distribution of the $\delta$-dimensional DLG process $R(t)$ defined by $(2.2)$, where $\alpha<0$, is $m$-dimensional $\Gamma$, i.e.

$$
\left(R^{(\delta)}\left(t_{1}\right), \ldots, R^{(\delta)}\left(t_{m}\right)\right) \sim \Gamma\left(\frac{\delta}{2}, 2 \Sigma\right)
$$


where $\Sigma$ is the $m \times m$ variance-covariance matrix of the accompanying Gaussian random vector $\left(X\left(t_{1}\right), \ldots\right.$, $\left.X\left(t_{m}\right)\right)$ and $X(t)$ is the stationary OU process defined by

$$
X(t)=\sigma \int_{-\infty}^{t} \mathrm{e}^{\alpha(t-s)} \mathrm{d} B(s)
$$

(See the Appendix for the multi-dimensional $\Gamma$ distribution.)

Proof. Because of (2.12), the same convolutional power rule connects the $m$-dimensional distributions of the vectors $\left(R_{\delta x}^{(\delta)}\left(t_{1}+s\right), \ldots, R_{\delta x}^{(\delta)}\left(t_{m}+s\right)\right)$ and $\left(R_{x}^{(1)}\left(t_{1}+s\right), \ldots, R_{x}^{(1)}\left(t_{m}+s\right)\right)$. Letting $s \rightarrow \infty$, we get the power rule for the $m$-dimensional stationary distributions, i.e. denoting the stationary distribution of $\left(R^{(\delta)}\left(t_{1}\right), \ldots, R^{(\delta)}\left(t_{m}\right)\right)$ by $P_{\underline{t}}^{(\delta)}$ we have

$$
P_{\underline{t}}^{(\delta)}=\left(P_{\underline{t}}^{(1)}\right)^{\circledast \delta}
$$

Since $P_{\underline{t}}^{(1)}$ is the distribution of a vector the components of which are the squares of the components of a homoscedastic Gauss-Markov vector, equation $(2.20)$ implies that $P_{\underline{t}}^{(\delta)}$ is the $m$-dimensional $\Gamma\left(\frac{\delta}{2}, 2 \Sigma\right)$ distribution.

Remark 2. The fact that the above vector $\left(R^{(\delta)}\left(t_{1}\right), \ldots, R^{(\delta)}\left(t_{m}\right)\right)$ has the multi-dimensional $\Gamma\left(\frac{\delta}{2}, 2 \Sigma\right)$ distribution means a new proof for Theorem 1 in [14] about the infinitely divisibility of the $\Gamma\left(\frac{1}{2}, 2 \Sigma\right)$ distribution. See also the Appendix.

The following theorem is the stationary counterpart of Proposition 3, and it is a direct consequence of (2.20). It is probably the most useful trait of the stationary DLG process, because it makes possible to derive properties of the $\delta$-dimensional DLG process from those of the simpler one-dimensional process.

Theorem 3. The distributions on $\mathcal{C}[0, T]$ of the $\delta$-dimensional and of the 1-dimensional stationary DLG processes, i.e. $P^{(\delta)}$ and $P^{(1)}$ are connected by the power rule

$$
P^{(\delta)}=\left(P^{(1)}\right)^{\circledast \delta}
$$

$\delta>0$. Here $\circledast \delta$ denotes the convolution power, defined via the same convolution power of the finite dimensional distributions.

Remark 3. Clearly, Theorem 3 implies that the stationary DLG process is infinitely divisible.

Remark 4. A useful observation found in [7] is that for $\alpha<0$ the right-hand side of (2.16) is just the characteristic function of the mixture of $\Gamma$ distributions by Poissonian weights, namely,

$$
R_{x}^{(\delta)}(t) \sim \Gamma\left(\frac{\delta}{2}+\eta, \frac{\sigma^{2}\left(1-\mathrm{e}^{2 \alpha t}\right)}{-\alpha}\right), \text { where } \eta \sim \operatorname{Poi}\left(\frac{-\alpha x}{\sigma^{2}\left(\mathrm{e}^{-2 \alpha t}-1\right)}\right) .
$$

The proof is a simple and short calculation, so we omit it. Equation (2.21) provides the simulation of the DLG process once a discrete set of times has been chosen.

\subsection{Cumulants, characteristic functional and spectra of the DLG process}

Let $R(t)$ be the stationary DLG process. We will consider $R(t)$ on an interval $[0, T]$ where $T>0$ is arbitrary but fixed.

We are now interested in the cumulants of finite-dimensional projections, and characteristic functional of the measure induced on $\mathcal{C}[0, T]$ by the stationary DLG process $R(t)$. Let us make it clear what we mean by the characteristic functional of a process $Z(t)$ with trajectories a.s. in $\mathcal{C}[0, T]$. We will also call it the characteristic functional of the measure generated on $\mathcal{C}[0, T]$ by process $Z(t)$. 
Notation 3. Let functional var denote the total variation of functions on $[0, T]$, and let $N B V[0, T] \doteq$ $\{G: G(0)=0, \operatorname{var}(G)<\infty\}$, i.e. $N B V[0, T]=\mathcal{C}[0, T]^{*}$, the dual space of $\mathcal{C}[0, T]$, the space of all continuous linear functionals on $\mathcal{C}[0, T]$. We will denote the characteristic functional of process $Z(t)$ with trajectories a.s. in $\mathcal{C}[0, T]$ by

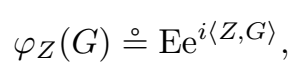

$G \in N B V[0, T]$, where

$$
\langle Z, G\rangle \stackrel{\circ}{=} \int_{0}^{T} Z(t) \mathrm{d} G(t)
$$

The following notations are needed for the sake of brevity:

Notation 4. Let us denote the set of all permutations of the elements of a set $A$ by $\operatorname{Perm}(A)$.

Notation 5. Let $m \in\{2,3,4, \ldots\}, \tau=\left(i_{1}, \ldots, i_{m-1}\right) \in \operatorname{Perm}(2,3, \ldots, m), \underline{t}=\left(t_{1}, \ldots, t_{m}\right) \in[0, T]^{m}$. Then

$$
D_{\tau}(\underline{t}) \stackrel{\circ}{=}\left|t_{i_{1}}-t_{1}\right|+\left|t_{i_{2}}-t_{i_{1}}\right|+\cdots+\left|t_{i_{m-1}}-t_{i_{m-2}}\right|+\left|t_{1}-t_{i_{m-1}}\right| .
$$

For $m=1$ define $D_{\tau}(t) \stackrel{\circ}{=} 0, t \in[0, T]$.

In particular, if $m=2$,

$$
D_{\tau}\left(t_{1}, t_{2}\right)=2\left|t_{1}-t_{2}\right|
$$

and for $m=3$,

$$
D_{\tau}\left(t_{1}, t_{2}, t_{3}\right)=2\left(\max \left(t_{1}, t_{2}, t_{3}\right)-\min \left(t_{1}, t_{2}, t_{3}\right)\right)=2 \operatorname{diam}\left(t_{1}, t_{2}, t_{3}\right) .
$$

Theorem 4. The cumulant of the m-dimensional distribution of the stationary DLG process $R(t)$ defined by (2.2) is

$$
\operatorname{cum}\left(R\left(t_{1}\right), \ldots, R\left(t_{m}\right)\right)= \begin{cases}\frac{\delta}{2}\left(\frac{-\alpha}{\sigma^{2}}\right)^{-m}, & \text { if } m=1 \\ \frac{\delta}{2}\left(\frac{-\alpha}{\sigma^{2}}\right)^{-m} \sum_{\tau \in \operatorname{Perm}(2, \ldots, m)} \mathrm{e}^{\alpha D_{\tau}\left(t_{1}, \ldots, t_{m}\right)}, & \text { if } m \geqslant 2 .\end{cases}
$$

In a neighborhood of $0 \in N B V[0, T]$ the logarithm of the characteristic functional of $R$ can be put into the following form.

$$
\log \varphi_{R}(G)=\frac{\delta}{2} \sum_{m=1}^{\infty} \frac{i^{m}}{m}\left(\frac{-\alpha}{\sigma^{2}}\right)^{-m} \int_{[0, T]^{m}} \mathrm{e}^{\alpha D_{\tau_{0}}(\underline{t})} d G\left(t_{1}\right) \ldots \mathrm{d} G\left(t_{m}\right)
$$

for $G \in N B V[0, T]: \operatorname{var}(G)<-\alpha / \sigma^{2}$, where $\tau_{0}$ is the identity permutation.

Proof. As we have seen, Theorem 3 permits of deriving the distributional properties of the $\delta$-dimensional DLG process from those of the one-dimensional process. Thus, in effect we have to deal only with the one-dimensional stationary DLG process $R(t)=X^{2}(t)$, where $X(t)$ is the stationary OU process $(2.19)$.

Now consider the cumulants. For $m=1$,

$$
\operatorname{cum}(R(t))=\operatorname{E} R(t)=\frac{1}{2}\left(\frac{-\alpha}{\sigma^{2}}\right)^{-1}
$$


because $R(t) \sim \Gamma\left(1 / 2,-\sigma^{2} / \alpha\right)$ by Proposition 6 . The right-hand side of $(2.24)$ is the first line of the right-hand side of (2.22) for $\delta=1$. For $m \geqslant 2$, by Proposition 27 in the Appendix, we have

$$
\begin{aligned}
\operatorname{cum}\left(R\left(t_{1}\right), \ldots, R\left(t_{m}\right)\right) & =2^{m-1} \sum_{\left(i_{1}, \ldots, i_{m-1}\right) \in \operatorname{Perm}(2, \ldots, m)} \operatorname{cov}\left(X\left(t_{1}\right), X\left(t_{i_{1}}\right)\right) \operatorname{cov}\left(X\left(t_{i_{1}}\right), X\left(t_{i_{2}}\right)\right) \cdots \\
\times \operatorname{cov}\left(X\left(t_{i_{m-2}}\right), X\left(t_{i_{m-1}}\right)\right) \operatorname{cov}\left(X\left(t_{i_{m-1}}\right), X\left(t_{1}\right)\right) & 2^{m-1} \sum_{\tau \in \operatorname{Perm}(2, \ldots, m)}\left(\frac{\sigma^{2}}{-2 \alpha}\right)^{m} \mathrm{e}^{\alpha D_{\tau}(\underline{t})},
\end{aligned}
$$

which is the second line of the right-hand side of (2.22) for $\delta=1$. We remark that one can get the same result by using formula (2.9) in [40], which is a direct expression for the joint cumulants of multiple Wiener-Itô integrals.

Let us now calculate the characteristic functional. Or rather its logarithm, inasmuch as the cumulants will be used in the series expansion.

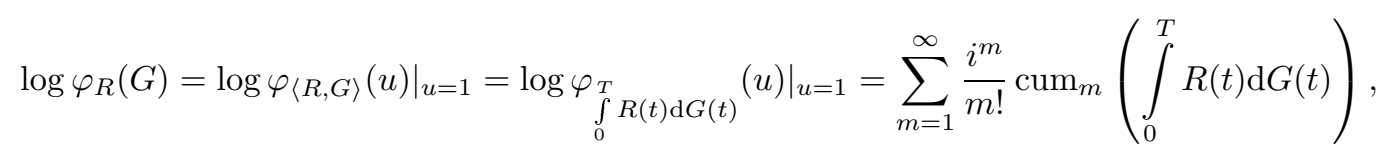

where $\operatorname{cum}_{m}$ denotes the cumulant of order $m$. Since

$$
\begin{aligned}
\operatorname{cum}_{m}\left(\int_{0}^{T} R(t) \mathrm{d} G(t)\right) & =\operatorname{cum}(\underbrace{\int_{0}^{T} R(t) \mathrm{d} G(t), \ldots, \int_{0}^{T} R(t) \mathrm{d} G(t)}_{m \text { in all }}) \\
& =\underbrace{T}_{m \text { in all }} \ldots \int_{0}^{T} \operatorname{cum}\left(R\left(t_{1}\right), \ldots R\left(t_{m}\right)\right) \mathrm{d} G\left(t_{1}\right) \ldots \mathrm{d} G\left(t_{m}\right) \\
& =\int_{[0, T]^{m}}^{\frac{\delta}{2}}\left(\frac{-\alpha}{\sigma^{2}}\right)^{-m} \sum_{\tau \in \operatorname{Perm}(2, \ldots, m)} \mathrm{e}^{\alpha D_{\tau}\left(t_{1}, \ldots, t_{m}\right)} \mathrm{d} G\left(t_{1}\right) \ldots \mathrm{d} G\left(t_{m}\right) \\
& =\frac{\delta}{2}\left(\frac{-\alpha}{\sigma^{2}}\right)^{-m}(m-1) ! \int_{[0, T]^{m}} \mathrm{e}^{\alpha D_{\tau_{0}}(\underline{t})} \mathrm{d} G\left(t_{1}\right) \ldots \mathrm{d} G\left(t_{m}\right),
\end{aligned}
$$

for $m \geqslant 2$, and the left-hand side equals the right-hand side also for $m=1$. In (2.26) the change of the order of the cumulant operator and the integrals can be justified, e.g. by the following way. Let us express the cumulant via moments. Then in each term of products of moments one can change the order of the expectations and the multiple integrals, because the conditions of the Fubini theorem are fulfilled. Then changing back the expression via moments to cumulant one gets the second row of (2.26).

Returning to (2.25) and using (2.26), we have the result as in (2.23). It is easy to see that the restriction $G \in N B V[0, T]: \operatorname{var}(G)<-\alpha / \sigma^{2}$ is enough for the series on the right-hand side of (2.25) to be convergent.

Now consider the stationary DLG process $R(t)$ on the whole line, i.e. $t \in \mathbb{R}$. We will be interested in the spectra of the process, namely, the second and third order ones, i.e. the spectrum and the bispectrum, respectively. Of the higher order spectra it is the bispectrum that has the least order. The notion of higher order spectrum arises from Brillinger, see [3].

Definition 8. Let $m \in\{2,3,4, \ldots\}$. Let $X(t), t \in \mathbb{R}$, be a stationary process with finite absolute moments of order $m$. Assume that the joint cumulant function, taken as a function of $m-1$ variables $\underline{t}_{(m-1)} \stackrel{\circ}{=}$ 
$\left(t_{1}, \ldots, t_{m-1}\right)$, is integrable, i.e.

$$
\int_{\mathbb{R}^{m-1}}\left|\operatorname{cum}\left(X(0), X\left(t_{1}\right), \ldots, X\left(t_{m-1}\right)\right)\right| \mathrm{d} \underline{t}_{(m-1)}<\infty
$$

and that the $(m-1)$-variable Fourier transform

$$
S_{m, X}\left(\underline{\omega}_{(m-1)}\right) \doteq\left(\frac{1}{2 \pi}\right)^{m-1} \int_{\mathbb{R}^{m-1}} \exp \left(-i \sum_{j=1}^{m-1} t_{j} \omega_{j}\right) \operatorname{cum}\left(X(0), X\left(t_{1}\right), \ldots, X\left(t_{m-1}\right)\right) \mathrm{d} \underline{t}_{(m-1)},
$$

where $\underline{\omega}_{(m-1)} \stackrel{\circ}{=}\left(\omega_{1}, \ldots, \omega_{m-1}\right)$, is also integrable, i.e.

$$
\int_{\mathbb{R}^{m-1}}\left|S_{m, X}\left(\underline{\omega}_{(m-1)}\right)\right| \mathrm{d} \underline{\omega}_{(m-1)}<\infty .
$$

Then $S_{m, X}\left(\underline{\omega}_{(m-1)}\right)$ is called the $m$-th order spectrum of process $X(t)$. For $m=2$ and $m=3$ the $m$-th order spectrum is called spectrum and bispectrum, respectively.

Remark 5. The spectrum of order $m \geqslant 3$ is generally, complex-valued, and so is the bispectrum. Only the second order spectrum is always real-valued.

Lemma 2. For each $m \in\{2,3,4, \ldots\}$ the cumulant function of the stationary DLG process $R(t)$, taken as a function of $m-1$ variables, is integrable, i.e.

$$
\int_{\mathbb{R}^{m-1}}\left|\operatorname{cum}\left(R(0), R\left(t_{1}\right), \ldots, R\left(t_{m-1}\right)\right)\right| \mathrm{d} \underline{t}_{(m-1)}<\infty .
$$

Proof. By (2.22), performing an integral transformation, we have

$$
\begin{aligned}
\int_{\mathbb{R}^{m-1}} \mid & \operatorname{cum}\left(R(0), R\left(t_{1}\right), \ldots, R\left(t_{m-1}\right)\right) \mid \mathrm{d} \underline{t}_{(m-1)} \\
= & \frac{\delta}{2}\left(\frac{-\alpha}{\sigma^{2}}\right)^{-m} \sum_{\tau \in \operatorname{Perm}(1, \ldots, m-1)} \int_{\mathbb{R}^{m-1}} \mathrm{e}^{\alpha D_{\tau}\left(0, t_{1}, \ldots, t_{m-1}\right)} \mathrm{d} \underline{t}_{(m-1)} . \\
= & \frac{\delta}{2}\left(\frac{-\alpha}{\sigma^{2}}\right)^{-m}(m-1) ! \int_{\mathbb{R}^{m-1}} \exp \left[\alpha\left(\sum_{j=1}^{m-1}\left|s_{j}\right|+\left|\sum_{j=1}^{m-1} s_{j}\right|\right)\right] \mathrm{d} \underline{s}_{(m-1)} \\
\leqslant & \frac{\delta}{2}\left(\frac{-\alpha}{\sigma^{2}}\right)^{-m}(m-1) ! \int_{\mathbb{R}^{m-1}} \exp \left(\alpha \sum_{j=1}^{m-1}\left|s_{j}\right|\right) \mathrm{d} \underline{s}_{(m-1)}<\infty,
\end{aligned}
$$

where we also utilized the negativity of $\alpha$ in both of the inequalities.

Proposition 8. The spectrum of the stationary DLG process $R(t)$ exists, and it is

$$
S_{2, R}(\omega)=\frac{1}{2 \pi} \cdot \frac{\delta}{2}\left(\frac{-\alpha}{\sigma^{2}}\right)^{-2} \frac{-4 \alpha}{\omega^{2}+4 \alpha^{2}} .
$$


Proof. By Lemma 2, Definition 8 and formula (2.22), the Fourier transform of the second order cumulant function with one variable is,

$$
\begin{aligned}
S_{2, R}(\omega) & =\frac{1}{2 \pi} \int_{\mathbb{R}} \mathrm{e}^{-i t \omega} \operatorname{cum}(R(0), R(t)) \mathrm{d} t \\
& =\frac{1}{2 \pi} \cdot \frac{\delta}{2}\left(\frac{-\alpha}{\sigma^{2}}\right)^{-2} \int_{\mathbb{R}} \mathrm{e}^{-i t \omega+2 \alpha|t|} \mathrm{d} t \\
& =\frac{1}{2 \pi} \cdot \frac{\delta}{2}\left(\frac{-\alpha}{\sigma^{2}}\right)^{-2} \frac{-4 \alpha}{\omega^{2}+4 \alpha^{2}} .
\end{aligned}
$$

$S_{2, R}(\omega)$ is also integrable, thus it is really a spectrum.

Remark 6. By the previous proposition the spectrum of the stationary DLG process $R(t)$ is the same as that of an Ornstein-Uhlenbeck $(\mathrm{AR}(1))$ process.

Notation 6. The symmetrization operator sym is defined by the formula

$$
\operatorname{sym}_{\substack{\left.\left(\underline{\omega}_{(3)}\right) \\ \underline{\omega}_{(3)}\right)}}\left(g\left(\omega_{1}, \omega_{2}, \omega_{3}\right)\right)=\frac{1}{3 !} \sum_{\left(i_{1}, i_{2}, i_{3}\right) \in \operatorname{Perm}(1,2,3)} g\left(\omega_{i_{1}}, \omega_{i_{2}}, \omega_{i_{3}}\right) .
$$

Proposition 9. The bispectrum of the stationary DLG process $R(t)$ exists, and it is

$$
S_{3, R}\left(\underline{\omega}_{(2)}\right)=\left(\frac{1}{2 \pi}\right)^{2} \delta\left(\frac{-\alpha}{\sigma^{2}}\right)^{-3} 3 ! \underset{\left(\underline{\omega}_{(3)}\right)}{\operatorname{sym}}\left(\frac{1}{-i \omega_{1}-2 \alpha} \cdot \frac{1}{i \omega_{2}-2 \alpha}\right)
$$

where $\omega_{1}+\omega_{2}+\omega_{3}=0$. In another form $(2.28)$ is written as

$$
S_{3, R}\left(\underline{\omega}_{(2)}\right)=\left(\frac{1}{2 \pi}\right)^{2} 48 \delta\left(\frac{-\alpha}{\sigma^{2}}\right)^{-3} \frac{\alpha^{2}\left(\omega_{1} \omega_{2}+\omega_{1}^{2}+\omega_{2}^{2}+12 \alpha^{2}\right)}{\left(\omega_{1}^{2}+4 \alpha^{2}\right)\left(\omega_{2}^{2}+4 \alpha^{2}\right)\left(\left(\omega_{1}+\omega_{2}\right)^{2}+4 \alpha^{2}\right)} .
$$

Hence, $S_{3, R}\left(\underline{\omega}_{(2)}\right)$ has the extraordinary property that it is real-valued moreover it is positive.

Proof. By Lemma 2, Definition 8 and formula (2.22), the Fourier transform of the second order cumulant function with two variables is,

$$
\begin{aligned}
S_{3, R}\left(\underline{\omega}_{(2)}\right) & =\left(\frac{1}{2 \pi}\right)^{2} \int_{\mathbb{R}^{2}} \mathrm{e}^{-i\left(t_{1} \omega_{1}+t_{2} \omega_{2}\right)} \operatorname{cum}\left(R(0), R\left(t_{1}\right), R\left(t_{2}\right) \mathrm{d} \underline{t}\right. \\
& =\left(\frac{1}{2 \pi}\right)^{2} \frac{\delta}{2}\left(\frac{-\alpha}{\sigma^{2}}\right)^{-3} \int_{\mathbb{R}^{2}} \mathrm{e}^{-i\left(t_{1} \omega_{1}+t_{2} \omega_{2}\right)} \sum_{\tau \in \operatorname{Perm}(1,2)} \mathrm{e}^{\alpha D_{\tau}\left(0, t_{1}, t_{2}\right)} \mathrm{d} \underline{t} \\
& =\left(\frac{1}{2 \pi}\right)^{2} \frac{\delta}{2}\left(\frac{-\alpha}{\sigma^{2}}\right)^{-3} 2 ! \int_{\mathbb{R}^{2}} \mathrm{e}^{-i\left(t_{1} \omega_{1}+t_{2} \omega_{2}\right)+\alpha\left(\left|t_{1}\right|+\left|t_{2}-t_{1}\right|+\left|t_{2}\right|\right)} \mathrm{d} \underline{t} \\
& =\left(\frac{1}{2 \pi}\right)^{2} \frac{\delta}{2}\left(\frac{-\alpha}{\sigma^{2}}\right)^{-3} 2 ! \int_{\mathbb{R}^{2}} \mathrm{e}^{-i\left(s_{1}\left(\omega_{1}+\omega_{2}\right)+s_{2} \omega_{2}\right)+\alpha\left(\left|s_{1}\right|+\left|s_{2}\right|+\left|s_{1}+s_{2}\right|\right)} \mathrm{d} \underline{s} .
\end{aligned}
$$


Breaking up the double integral according to the six parts of the plane defined by the axes and the line $y=-x$, we have

$$
\int_{\mathbb{R}^{2}} \mathrm{e}^{-i\left(s_{1}\left(\omega_{1}+\omega_{2}\right)+s_{2} \omega_{2}\right)+\alpha\left(\left|s_{1}\right|+\left|s_{2}\right|+\left|s_{1}+s_{2}\right|\right)} \mathrm{d} \underline{s}=3 ! \underset{\left(\underline{\omega}_{(3)}\right)}{\operatorname{sym}}\left(\frac{1}{-i \omega_{1}-2 \alpha} \cdot \frac{1}{i \omega_{2}-2 \alpha}\right) .
$$

Continuing (2.30), we have for the Fourier transform

$$
\begin{aligned}
S_{3, R}\left(\underline{\omega}_{(2)}\right) & =\left(\frac{1}{2 \pi}\right)^{2} \delta\left(\frac{-\alpha}{\sigma^{2}}\right)^{-3} 3 ! \operatorname{sym}_{\left(\underline{\omega}_{(3)}\right)}\left(\frac{1}{-i \omega_{1}-2 \alpha} \cdot \frac{1}{i \omega_{2}-2 \alpha}\right) \\
& =\left(\frac{1}{2 \pi}\right)^{2} \delta\left(\frac{-\alpha}{\sigma^{2}}\right)^{-3} 3 ! \frac{8 \alpha^{2}\left(\omega_{1} \omega_{2}+\omega_{1}^{2}+\omega_{2}^{2}+12 \alpha^{2}\right)}{\left(\omega_{1}^{2}+4 \alpha^{2}\right)\left(\omega_{2}^{2}+4 \alpha^{2}\right)\left(\left(\omega_{1}+\omega_{2}\right)^{2}+4 \alpha^{2}\right)} .
\end{aligned}
$$

We remark that though it does not show at first sight, the numerator of the fraction in the last row is symmetric in the variables $\omega_{1}, \omega_{2}, \omega_{3}$. Now, if $\omega_{1}$ and $\omega_{2}$ are both nonnegative or both negative, the fraction in the last row of $(2.31)$ can be bounded as

$$
\begin{aligned}
0 & <\frac{8 \alpha^{2}\left(\omega_{1} \omega_{2}+\omega_{1}^{2}+\omega_{2}^{2}+12 \alpha^{2}\right)}{\left(\omega_{1}^{2}+4 \alpha^{2}\right)\left(\omega_{2}^{2}+4 \alpha^{2}\right)\left(\left(\omega_{1}+\omega_{2}\right)^{2}+4 \alpha^{2}\right)} \\
& \leqslant \frac{8 \alpha^{2}\left(\left(\omega_{1}+\omega_{2}\right)^{2}+12 \alpha^{2}\right)}{\left(\omega_{1}^{2}+4 \alpha^{2}\right)\left(\omega_{2}^{2}+4 \alpha^{2}\right)\left(\left(\omega_{1}+\omega_{2}\right)^{2}+4 \alpha^{2}\right)} \\
& =\frac{8 \alpha^{2}}{\left(\omega_{1}^{2}+4 \alpha^{2}\right)\left(\omega_{2}^{2}+4 \alpha^{2}\right)}+\frac{64 \alpha^{4}}{\left(\omega_{1}^{2}+4 \alpha^{2}\right)\left(\omega_{2}^{2}+4 \alpha^{2}\right)\left(\left(\omega_{1}+\omega_{2}\right)^{2}+4 \alpha^{2}\right)} \\
& \leqslant \frac{8 \alpha^{2}}{\left(\omega_{1}^{2}+4 \alpha^{2}\right)\left(\omega_{2}^{2}+4 \alpha^{2}\right)}+\frac{64 \alpha^{4}}{\left(\omega_{1}^{2}+4 \alpha^{2}\right)\left(\omega_{2}^{2}+4 \alpha^{2}\right) 4 \alpha^{2}}
\end{aligned}
$$

and so $S_{3, R}\left(\underline{\omega}_{(2)}\right)$ is integrable on the first and third quarters of the plane. If $\omega_{1}$ and $\omega_{2}$ have different signs, then

$$
\begin{aligned}
0< & \frac{8 \alpha^{2}\left(\omega_{1} \omega_{2}+\omega_{1}^{2}+\omega_{2}^{2}+12 \alpha^{2}\right)}{\left(\omega_{1}^{2}+4 \alpha^{2}\right)\left(\omega_{2}^{2}+4 \alpha^{2}\right)\left(\left(\omega_{1}+\omega_{2}\right)^{2}+4 \alpha^{2}\right)} \\
\leqslant & \frac{8 \alpha^{2}\left(\omega_{1}^{2}+\omega_{2}^{2}+12 \alpha^{2}\right)}{\left(\omega_{1}^{2}+4 \alpha^{2}\right)\left(\omega_{2}^{2}+4 \alpha^{2}\right)\left(\left(\omega_{1}+\omega_{2}\right)^{2}+4 \alpha^{2}\right)} \\
= & \frac{8 \alpha^{2}}{\left(\omega_{2}^{2}+4 \alpha^{2}\right)\left(\left(\omega_{1}+\omega_{2}\right)^{2}+4 \alpha^{2}\right)}+\frac{8 \alpha^{2}}{\left(\omega_{1}^{2}+4 \alpha^{2}\right)\left(\left(\omega_{1}+\omega_{2}\right)^{2}+4 \alpha^{2}\right)} \\
& \quad+\frac{\left(\omega_{1}^{2}+4 \alpha^{2}\right)\left(\omega_{2}^{2}+4 \alpha^{2}\right)\left(\left(\omega_{1}+\omega_{2}\right)^{2}+4 \alpha^{2}\right)}{8 \alpha^{2}}+\frac{8 \alpha^{2}}{\left(\omega_{1}^{2}+4 \alpha^{2}\right)\left(\left(\omega_{1}+\omega_{2}\right)^{2}+4 \alpha^{2}\right)}+\frac{32 \alpha^{4}}{\left(\omega_{1}^{2}+4 \alpha^{2}\right)\left(\omega_{2}^{2}+4 \alpha^{2}\right) 4 \alpha^{2}}
\end{aligned}
$$

and thus, $S_{3, R}\left(\underline{\omega}_{(2)}\right)$ is integrable on the second and fourth quarters of the plane, too. This means that $S_{3, R}\left(\underline{\omega}_{(2)}\right)$ is integrable on the whole plane $\mathbb{R}^{2}$, thus it is really a bispectrum. We have also proven that it is real-valued and positive. 


\section{Superposition of DLG PROCESSES AND its MUltifractal Limit PROCESS}

\subsection{Superposition of random-parameter stationary DLG processes (SDLG process)}

Derivation of LRD by superposing short range dependent processes is not new in the literature. Here are some papers on this subject: reference [28] showed that superposition of many sources exhibiting the infinite variance syndrome can result in LRD. Reference [13] aggregated random coefficient AR(1) time series, while $[27,32]$ superposed renewal processes and alternating renewal processes with heavy tailed interarrival time distributions to get LRD. A model similar to the latter was considered in [39, 42], aggregating independent $\mathrm{ON} / \mathrm{OFF}$ processes with heavy tailed ON or OFF time periods to achieve LRD. References $[5,6]$ pointed out that the Riemann-Liouville fractional integral operator can be represented as a mixture of AR(1) operators. Reference [16] considered 1) superposition of random-coefficient independent OU processes; 2) mixture of random-coefficient OU processes with common input and 3) superposition of random-intensity Poisson alternating 0-1 reward processes, and the result was a LRD process, called GMOU process in all the three cases. Reference [1] studied the superposition of OU processes with Lévy process input, and under specified conditions, obtained a LRD process as a result.

The superposition model to be exposed in this subsection is similar to the first model of [16]; however the component processes to be superposed are not OU but another sort of AR(1) processes. They are DLG processes. Going into details, we will show that if we take a sequence of series of independent stationary DLG processes

$$
\begin{array}{ccc}
R_{1,1}(t) & & \\
R_{2,1}(t), & R_{2,2}(t) & \\
\vdots & & \\
R_{n, 1}(t), & \ldots, & R_{n, n}(t)
\end{array}
$$

with properly chosen random parameters, the row sums will converge in a certain sense to a limit process $Y$, i.e.

$$
Z_{n} \stackrel{\circ}{=} \sum_{k=1}^{n} R_{n, k} \underset{n \rightarrow \infty}{\longrightarrow} Y .
$$

As we will see, the characteristic functional of the limit process $Y(t)$, called Superposition of DLG processes (SDLG), is some weighted geometric mean of the characteristic functionals of stationary DLG processes. The latter processes are called component processes. As an outcome, both the joint cumulants and the spectra of the SDLG process $Y(t)$ are mixtures of the joint cumulants and the spectra, respectively, of the component processes. This fact makes it possible to calculate the cumulants and spectra of process $Y(t)$ from those of the component processes. Examining the cumulants, one can observe that the SDLG process $Y(t)$ has fractal-like properties in that its second order cumulant structure is asymptotically the same as that of the continuous-time fractional Gaussian noise. Moreover, it is LRD, and its centered integral process is asymptotically multifractal. The one-dimensional distribution of $Y(t)$ is also worthy of note: it is $\Gamma$. One can go further and take a limit process of series of SDLG processes which is already exact multifractal, see Section 3.2. An additional remarkable attribute is that the bispectrum of $Y(t)$ is real-valued moreover it is positive. The former property of the bispectrum involves some reversibility-like feature of the 3-dimensional distribution, see Remark 17.

Let us see the construction in details. Assume that there is given a sequence of parameters $\alpha_{k}, k \in \mathbb{N}$, such that they are independent random variables on a probability space $\left(\Omega_{A}, \mathcal{F}_{A}, P_{A}\right)$, and $-2 \alpha_{k} \sim \Gamma(3-2 H, 1 / \lambda)$, $k \in \mathbb{N}$, where $\lambda>0$ and $1 / 2<H<1$ are constants. Assume moreover that the parameters $\sigma_{k}^{2}$ depend on parameters $\alpha_{k}$, such that $\sigma_{k}^{2}=-\alpha_{k} \sigma_{0}^{2}$, where $\sigma_{0}^{2}>0$ is a constant. Furthermore let the parameters $\mu_{n} \stackrel{\circ}{=} \mu_{0} / n, n \in \mathbb{N}$, where $\mu_{0}>0$ is a constant. Thus parameters $\alpha_{k}$ and $\sigma_{k}$ are random variables, parameters $\mu_{n}$ are deterministic, and the parameters $\delta_{n, k} \stackrel{\circ}{=} \mu_{n} / \sigma_{k}^{2}$ (see (2.3)) are random variables too. 
Let the probability space $\left(\Omega_{B}, \mathcal{F}_{B}, P_{B}\right)^{2}$ be independent of $\left(\Omega_{A}, \mathcal{F}_{A}, P_{A}\right)^{1}$. Let the sequence of series (3.1) of the stationary DLG processes be defined $P_{A}$ a.s. on $\left(\Omega_{B}, \mathcal{F}_{B}, P_{B}\right)$, specifically, the process $R_{n}, k(t)$ with input BM $B_{k}(t)$ and parameters $\alpha_{k}, \sigma_{k}$, and $\mu_{n}$, for each $(n, k), n \in \mathbb{N}, k=1, \ldots, n$. Assume that the BMs $B_{k}(t), k \in \mathbb{N}$, are independent. Thus, we have

$$
\mathrm{d} R_{n, k}(t)=\left(\mu_{n}+2 \alpha_{k} R_{n, k}(t)\right) \mathrm{d} t+2 \sigma_{k} \sqrt{R_{n, k}(t)} \mathrm{d} B_{k}(t), \quad P_{A} \text { a.s. }
$$

$n \in \mathbb{N}, k=1, \ldots, n$.

Now we summarize the above conditions.

Condition 1. $\lambda>0,1 / 2<H<1, \sigma_{0}^{2}>0$ and $\mu_{0}>0$ are constants.

Condition 2. $\left(\Omega_{A}, \mathcal{F}_{A}, P_{A}\right)$ and $\left(\Omega_{B}, \mathcal{F}_{B}, P_{B}\right)$ are independent probability spaces.

Condition 3. $-2 \alpha_{k} \sim \Gamma(3-2 H, 1 / \lambda), k \in \mathbb{N}$, are independent random variables on $\left(\Omega_{A}, \mathcal{F}_{A}, P_{A}\right)$.

Condition 4. $\sigma_{k}^{2} \stackrel{\circ}{=}-\alpha_{k} \sigma_{0}^{2}, k \in \mathbb{N}$.

Condition 5. $\mu_{n} \stackrel{\circ}{=} \mu_{0} / n, n \in \mathbb{N}$.

Condition 6. $B_{k}(t), k \in \mathbb{N}$, are independent Brownian motions on $\left(\Omega_{B}, \mathcal{F}_{B}, P_{B}\right)$.

Stressing it again, we have the stationary DLG processes $R_{n, k}(t)$ defined by (3.2). Specifically, $R_{1,1}(t)$ has the parameters $-2 \alpha_{1} \sim \Gamma(3-2 H, 1 / \lambda), \sigma_{1}^{2}=-\alpha_{1} \sigma_{0}^{2}$ and $\mu_{1}=\mu_{0}$.

Remark 7. Note that it is Condition 5 only that accounts for our having not simply a series but a sequence of series of DLG processes.

Notation 7. Let us define for each $n \in \mathbb{N}$ the process $Z_{n}(t)$ to be the sum of the $n$-th row of (3.1), i.e.

$$
Z_{n}(t) \stackrel{n}{=} \sum_{k=1}^{n} R_{n, k}(t)
$$

$n \in \mathbb{N}$.

Remember that due to Proposition $6, R_{n, k}(t) \sim \Gamma\left(\delta_{n, k} / 2,-\sigma_{k}^{2} / \alpha_{k}\right)$, where $\delta_{n, k}=\mu_{n} / \sigma_{k}^{2}$, i.e.

$$
R_{n, k}(t) \sim \Gamma\left(\frac{\mu_{0}}{2 n \sigma_{k}^{2}}, \sigma_{0}^{2}\right) .
$$

The first observation is that the one-dimensional limit distribution of $Z_{n}(t)$ remains $\Gamma$ as $n \rightarrow \infty$.

Proposition 10. For every fixed $t \in \mathbb{R}$ the distribution of the series of random variables $Z_{n}(t) P_{A}$ a.s. converges weakly to a $\Gamma$ distribution, that is to say,

$$
Z_{n}(t) \underset{n \rightarrow \infty}{\stackrel{w}{\longrightarrow}} \Gamma\left(\frac{\mu_{0} \lambda}{2 \sigma_{0}^{2}(1-H)}, \sigma_{0}^{2}\right), \quad P_{A} \text { a.s. }
$$

Proof. Because of the independence of processes $R_{n, k}(t), k=1, \ldots, n$, and Condition 4 , we have

$$
Z_{n}(t) \sim \Gamma\left(\frac{\mu_{0}}{n \sigma_{0}^{2}} \sum_{k=1}^{n} \frac{1}{-2 \alpha_{k}}, \sigma_{0}^{2}\right)
$$

Because of Condition 3, it follows, by the strong law of large numbers, that

$$
\frac{\mu_{0}}{n \sigma_{0}^{2}} \sum_{k=1}^{n} \frac{1}{-2 \alpha_{k}} \underset{n \rightarrow \infty}{\longrightarrow} \frac{\mu_{0}}{\sigma_{0}^{2}} \mathrm{E}_{A} \frac{1}{-2 \alpha_{k}}=\frac{\mu_{0}}{\sigma_{0}^{2}} \cdot \frac{\lambda}{2(1-H)}, \quad P_{A} \text { a.s. }
$$

where $\mathrm{E}_{A}$ means the expectation with respect to the distribution $P_{A}$. From (3.4) and (3.5) the statement follows straightaway.

\footnotetext{
${ }^{2}$ We use the subscript $B$ and $A$, to remind the reader of the Brownian motion input process and the parameter $\alpha$, respectively.
} 
Corollary 1. The SDLG process $Y(t)$, if it exists, has marginal distribution $\Gamma$, that is,

$$
Y(t) \sim \Gamma\left(\frac{\mu_{0} \lambda}{2 \sigma_{0}^{2}(1-H)}, \sigma_{0}^{2}\right),
$$

for all $t \geqslant 0$.

Remark 8. For each $n \in \mathbb{N}, P_{A}$ a.s. holds that the random process $Z_{n}(t)$ is $P_{B}$ a.s. continuous, i.e. $P_{A}$ a.s.: $Z_{n} \in \mathcal{C}[0, T] P_{B}$ a.s. Thus, for each $n \in \mathbb{N}, P_{A}$ a.s.: process $Z_{n}$ induces a measure $P_{Z_{n}}$ on $\mathcal{C}[0, T]$. The measure $P_{Z_{n}}$ is random in the sense that it depends on the realization $\left(\alpha_{1}, \ldots, \alpha_{n}\right)$. However, it would be inadequate to call it a "random measure", so we omit the attribute "random".

Before the main theorem in this section we need the following lemma:

Lemma 3. $P_{A}$ a.s. holds that the series of measures $P_{Z_{n}}, n \in \mathbb{N}$, mentioned in Remark 8 , is tight.

Proof. We premise that in this proof every statement depending on the realizations of $\alpha_{1}, \alpha_{2}, \alpha_{3}, \ldots$, will be meant $P_{A}$ a.s.

It follows from Proposition 7 and from the form of the characteristic function of the multi-dimensional $\Gamma$ distribution (see the Appendix) that the characteristic function of $R_{n, k}(t)-R_{n, k}(0)$ is

$$
\begin{aligned}
\varphi_{R_{n, k}(t)-R_{n, k}(0)}(u) & =\varphi_{R_{n, k}(t), R_{n, k}(0)}(u,-u) \\
& =\left|I-i u \sigma_{0}^{2}\left(\begin{array}{cc}
1 & 0 \\
0 & -1
\end{array}\right)\left(\begin{array}{cc}
1 & \mathrm{e}^{\alpha_{k}|t|} \\
\mathrm{e}^{\alpha_{k}|t|} & 1
\end{array}\right)\right|^{-\frac{\mu_{0}}{-2 n \sigma_{0}^{2} \alpha_{k}}} \\
& =\left(1+u^{2} \sigma_{0}^{4}\left(1-\mathrm{e}^{2 \alpha_{k}|t|}\right)\right)^{-\frac{\mu_{0}}{-2 n \sigma_{0}^{2} \alpha_{k}}} .
\end{aligned}
$$

Calculating the moments from the characteristic function by differentiation, we have

$$
\begin{aligned}
& \mathrm{E}_{B}\left(R_{n, k}(t)-R_{n, k}(0)\right)^{2}=\frac{2 \mu_{0} \sigma_{0}^{2}}{n} \cdot \frac{1-\mathrm{e}^{2 \alpha_{k}|t|}}{-2 \alpha_{k}}, \\
& \mathrm{E}_{B}\left(R_{n, k}(t)-R_{n, k}(0)\right)^{4}=12 \frac{\mu_{0}}{-2 n \sigma_{0}^{2} \alpha_{k}}\left(\frac{\mu_{0}}{-2 n \sigma_{0}^{2} \alpha_{k}}+1\right)\left(\left(1-\mathrm{e}^{2 \alpha_{k}|t|}\right) \sigma_{0}^{4}\right)^{2} .
\end{aligned}
$$

Hence

$$
\begin{aligned}
\mathrm{E}_{B}\left(Z_{n}(t)-Z_{n}(0)\right)^{4}= & \sum_{k=1}^{n} \mathrm{E}_{B}\left(R_{n, k}(t)-R_{n, k}(0)\right)^{4} \\
& +\sum_{k=1}^{n} \sum_{\ell=1}^{n} \mathrm{E}_{B}\left(R_{n, k}(t)-R_{n, k}(0)\right)^{2} \mathrm{E}_{B}\left(R_{n, \ell}(t)-R_{n, \ell}(0)\right)^{2} \\
\leqslant & \left(12 \frac{\mu_{0} \sigma_{0}^{6}}{n} \sum_{k=1}^{n}\left(\frac{\mu_{0}}{\sigma_{0}^{2} n}-2 \alpha_{k}\right)+\frac{4 \mu_{0}^{2} \sigma_{0}^{4}}{n^{2}} \sum_{k=1}^{n} \sum_{\ell=1}^{n} 1\right) t^{2} \\
= & \left(4 \mu_{0}^{2} \sigma_{0}^{4}\left(1+\frac{3}{n}\right)+\frac{12 \mu_{0} \sigma_{0}^{6}}{n} \sum_{k=1}^{n}\left(-2 \alpha_{k}\right)\right) t^{2} \\
\leqslant & \left(16 \mu_{0}^{2} \sigma_{0}^{4}+12 \mu_{0} \sigma_{0}^{6} K\left(\alpha_{1}, \alpha_{2}, \alpha_{3}, \ldots\right)\right) t^{2}
\end{aligned}
$$

for all $n \in \mathbb{N}$, where $K\left(\alpha_{1}, \alpha_{2}, \alpha_{3}, \ldots\right)$ is a constant bounding above the series $\frac{1}{n} \sum_{k=1}^{n}\left(-2 \alpha_{k}\right)$. Such a constant really exists, in agreement with the strong law of large numbers.

Process $Z_{n}(t)$ is stationary, thus it has stationary increments. Consequently, using (3.6), we have

$$
\mathrm{E}_{B}\left(Z_{n}(t+s)-Z_{n}(s)\right)^{4} \leqslant K_{1}\left(\alpha_{1}, \alpha_{2}, \alpha_{3}, \ldots\right) t^{2}
$$

for all $t, s>0$ and $n \in \mathbb{N}$. Thus the tightness condition of Kolmogorov is fulfilled. 
Now, in the main theorem of this section, we state the existence of the SDLG process $Y(t)$, and give a series expansion for its logarithmic characteristic functional.

Theorem 5. 1) $P_{A}$ a.s. holds that the series of measures $P_{Z_{n}}, n \in \mathbb{N}$, mentioned in Remark 8 , converges weakly to some measure $P_{Y}$, i.e. there exists an a.s. continuous process $Y(t)$ on $\left(\Omega_{B}, \mathcal{F}_{B}, P_{B}\right)$ such that

$$
Z_{n} \underset{n \rightarrow \infty}{\stackrel{w}{\longrightarrow}} Y \quad \text { in } \mathcal{C}[0, T], \quad P_{A} \text { a.s. }
$$

Distribution $P_{Y}$ and thus process $Y(t)$ do not depend on the realizations of $\alpha_{1}, \alpha_{2}, \alpha_{3}, \ldots$ any more. ( $Z_{n}$ and $P_{Z_{n}}$ do.)

2) For all $G \in N B V[0, T]$ such that $\operatorname{var}(G)<\sigma_{0}^{-2}$, the logarithm of the characteristic functional of process $Y(t)$ (or of $P_{Y}$ ) can be put into the form

$$
\log \varphi_{Y}(G)=\frac{\mu_{0} \lambda}{2 \sigma_{0}^{2}(1-H)} \sum_{m=1}^{\infty} \frac{i^{m}}{m} \sigma_{0}^{2 m} \int_{[0, T]^{m}}\left(1+\frac{D_{\tau_{0}}(\underline{t})}{2 \lambda}\right)^{2(H-1)} \mathrm{d} G\left(t_{1}\right) \ldots \mathrm{d} G\left(t_{m}\right),
$$

where $\tau_{0}$ is the identity permutation. (See Notation 5 on $D_{\tau_{0}}(\underline{t})$.)

3) The characteristic functional of process $Y(t)$ is the weighted geometric mean of the characteristic functionals of stationary $D L G$ component processes $R_{1,1}(t)$, where the weight measure is the $\Gamma(3-2 H, 1 / \lambda)$ distribution, see Condition 3. In other words, the logarithm of the characteristic functional of process $Y(t)$ is the mixture of logarithms of the characteristic functionals of component processes $R_{1,1}(t)$, i.e.

$$
\log \varphi_{Y}(G)=\mathrm{E}_{A} \log \varphi_{R_{1,1}}(G)
$$

for all $G \in N B V[0, T]$.

Proof. The proof of the first two statements is composed of four parts. The fifth part is the proof of the third statement of the theorem. We premise that in this proof every statement depending on the realizations of $\alpha_{1}, \alpha_{2}, \alpha_{3}, \ldots$, will be meant by $P_{A}$ a.s.

Part 1. Because of the independence of the elements inside the rows of (3.1), Theorem 4, formula (2.3), and Conditions 4 and 5, we have

$$
\begin{aligned}
\log \varphi_{Z_{n}}(G) & =\sum_{k=1}^{n} \log \varphi_{R_{n, k}}(G) \\
& =\sum_{m=1}^{\infty} \frac{i^{m}}{m} \mu_{0} \sigma_{0}^{2(m-1)} \int_{[0, T]^{m}} \frac{1}{n} \sum_{k=1}^{n}\left(\frac{1}{-2 \alpha_{k}} \mathrm{e}^{\alpha_{k} D_{\tau_{0}}(\underline{t})}\right) \mathrm{d} G\left(t_{1}\right) \ldots \mathrm{d} G\left(t_{m}\right),
\end{aligned}
$$

for $\operatorname{var}(G)<\sigma_{0}^{-2}$. Because of the strong law of large numbers and Condition 3,

$$
\frac{1}{n} \sum_{k=1}^{n}\left(\frac{1}{-2 \alpha_{k}} \mathrm{e}^{\alpha_{k} D_{\tau_{0}}(\underline{t})}\right) \underset{n \rightarrow \infty}{\longrightarrow} \mathrm{E}_{A}\left(\frac{1}{-2 \alpha_{k}} \mathrm{e}^{\alpha_{k} D_{\tau_{0}}(\underline{t})}\right)=\frac{\lambda}{2(1-H)}\left(1+\frac{D_{\tau_{0}}(\underline{t})}{2 \lambda}\right)^{2(H-1)} .
$$

Consequently

$$
\log \varphi_{Z_{n}}(G) \underset{n \rightarrow \infty}{\longrightarrow} \frac{\mu_{0} \lambda}{2 \sigma_{0}^{2}(1-H)} \sum_{m=1}^{\infty} \frac{i^{m}}{m} \sigma_{0}^{2 m} \int_{[0, T]^{m}}\left(1+\frac{D_{\tau_{0}}(\underline{t})}{2 \lambda}\right)^{2(H-1)} \mathrm{d} G\left(t_{1}\right) \ldots \mathrm{d} G\left(t_{m}\right),
$$

for $\operatorname{var}(G)<\sigma_{0}^{-2}$. The change of the order of the limit and the integral, and also the change of the order of the limit and the sum can be easily justified by the fact that we are considering the characteristic functionals only on the domain $\left\{G \in N B V[0, T]: \operatorname{var}(G)<\sigma_{0}^{-2}\right\}$. 
Part 2. As we found it simpler to deal directly with the characteristic functionals of measures on $\mathcal{C}[0, T]$ than to prove the convergence of finite-dimensional distributions, we will use the following well-known property of measures on complete separable metric spaces.

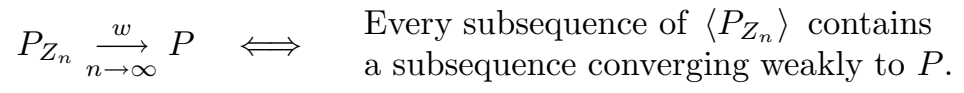

Let $\left\langle P_{Z_{n}^{-}}\right\rangle$be a subsequence of $\left\langle P_{Z_{n}}\right\rangle$. We have the following deduction chain.

Lemma $3 \Longrightarrow$ The series $\left\langle P_{Z_{n}^{-}}\right\rangle$is tight.

$\Longrightarrow$ The series $\left\langle P_{Z_{n}^{-}}\right\rangle$is relatively compact.

$\Longrightarrow \quad$ There exists a subsequence $\left\langle P_{Z} \widetilde{\widetilde{n}}\right\rangle$ of $\left\langle P_{Z_{n}}\right\rangle$, converging weakly to some measure, say $P$. $\Longrightarrow \quad \log \varphi_{Z \widetilde{\tilde{n}}}(G) \underset{n \rightarrow \infty}{\longrightarrow} \log \varphi_{P}(G)$ for all $G \in N B V[0, T]$,

where $\varphi_{Z \widetilde{\widetilde{n}}}(G)$ and $\varphi_{P}(G)$ is the characteristic functional of $P_{Z \widetilde{n}}$ and $P$, respectively.

From the last convergence and (3.9), we have

$$
\begin{aligned}
\log \varphi_{Z \widetilde{n}}(G) \underset{n \rightarrow \infty}{\longrightarrow} & \log \varphi_{P}(G) \\
& =\frac{\mu_{0} \lambda}{2 \sigma_{0}^{2}(1-H)} \sum_{m=1}^{\infty} \frac{i^{m}}{m} \sigma_{0}^{2 m} \int_{[0, T]^{m}}\left(1+\frac{D_{\tau_{0}}(\underline{t})}{2 \lambda}\right)^{2(H-1)} \mathrm{d} G\left(t_{1}\right) \ldots \mathrm{d} G\left(t_{m}\right),
\end{aligned}
$$

for $\operatorname{var}(G)<\sigma_{0}^{-2}$.

If one takes another subsequence $\left\langle P_{Z_{m}^{-}}\right\rangle$instead of $\left\langle P_{Z_{n}^{-}}\right\rangle$, then it follows in the same way that there exists a subsequence $\left\langle P_{Z_{\tilde{m}}}\right\rangle$ of $\left\langle P_{Z_{m}^{-}}\right\rangle$, and a probability measure $Q$ on $\mathcal{C}[0, T]$ such that

$$
\begin{aligned}
& \log \varphi_{Z} \approx \widetilde{\tilde{m}}(G) \underset{n \rightarrow \infty}{\longrightarrow} \log \varphi_{Q}(G) \\
& =\frac{\mu_{0} \lambda}{2 \sigma_{0}^{2}(1-H)} \sum_{m=1}^{\infty} \frac{i^{m}}{m} \sigma_{0}^{2 m} \int_{[0, T]^{m}}\left(1+\frac{D_{\tau_{0}}(\underline{t})}{2 \lambda}\right)^{2(H-1)} \mathrm{d} G\left(t_{1}\right) \ldots \mathrm{d} G\left(t_{m}\right),
\end{aligned}
$$

for $\operatorname{var}(G)<\sigma_{0}^{-2}$. It is simply the consequence

$$
\varphi_{P}(G)=\varphi_{Q}(G), \quad \text { for } \operatorname{var}(G)<\sigma_{0}^{-2},
$$

that concerns us in (3.11) and (3.12). By (3.10), what we have to prove is that $P=Q$.

Part 3. To prove that $P=Q$, it is enough to prove that all the finite-dimensional marginal distributions of the two measures are the same. In other words, we have to prove that all the finite-dimensional distributions of the a.s. continuous processes, say, $Y_{P}$ and $Y_{Q}$, are the same, where $Y_{P}$ and $Y_{Q}$ has distribution $P$ and $Q$, respectively.

Let $m \in \mathbb{N}$ and $t_{1}, \ldots, t_{m} \in[0, T]$ be arbitrary. We have to prove that the distribution of $\left(Y_{P}\left(t_{1}\right), \ldots\right.$, $\left.Y_{P}\left(t_{m}\right)\right)$ and that of $\left(Y_{Q}\left(t_{1}\right), \ldots, Y_{Q}\left(t_{m}\right)\right)$ on $\mathbb{R}^{m}$ are the same. To attain this it is enough to prove that all the linear combinations of their components have the same one-dimensional distribution, i.e.

$$
\sum_{k=1}^{m} \lambda_{k} Y_{P}\left(t_{k}\right) \sim \sum_{k=1}^{m} \lambda_{k} Y_{Q}\left(t_{k}\right)
$$

for all $\lambda_{1}, \ldots, \lambda_{m} \in \mathbb{R}$.

In (3.13) let us perform the substitution

$$
G \stackrel{\circ}{=} \sum_{k=1}^{m} \lambda_{k} \chi_{\left[t_{k}, T\right]},
$$


where

$$
|u|<\sigma_{0}^{-2}\left(\sum_{k=1}^{m}\left|\lambda_{k}\right|\right)^{-1}
$$

and $\chi$ denotes the characteristic (or indicator) function of the set in its subscript. This replacement is legitimate because obviously $\operatorname{var}(G)<\sigma_{0}^{-2}$. In this way we have

$$
\varphi \sum_{k=1}^{m} \lambda_{k} Y_{P}\left(t_{k}\right)(u)=\varphi_{P}(G)=\varphi_{Q}(G)=\varphi \sum_{k=1}^{m} \lambda_{k} Y_{Q}\left(t_{k}\right)(u)
$$

for the values of $u$ given by (3.15). Thus, in a neighborhood of $u=0$ the characteristic functions are equal, from which the equality of the moments of the two random variables in (3.14) follows. To complete the proof we have to prove that these moments determine the distribution uniquely.

Part 4. We have to prove that the moments of the random variable

$$
\sum_{k=1}^{m} \lambda_{k} Y_{P}\left(t_{k}\right)
$$

uniquely determine the distribution, and the proof is complete.

It is well known that the moments $M_{n}, n \in \mathbb{N}$, of a distribution, uniquely determine the distribution, if the absolute moments of even order, $\bar{M}_{2 n}, n \in \mathbb{N}$, satisfy the moment condition

$$
\varlimsup_{n \rightarrow \infty} \frac{\left(\bar{M}_{2 n}\right)^{\frac{1}{2 n}}}{2 n}<\infty .
$$

In our case

$$
\begin{aligned}
& \varlimsup_{n \rightarrow \infty} \frac{\left(\mathrm{E}\left|\sum_{k=1}^{m} \lambda_{k} Y_{P}\left(t_{k}\right)\right|^{2 n}\right)^{\frac{1}{2 n}}}{2 n}=\varlimsup_{n \rightarrow \infty}\left(\frac{1}{2 n}\left\|\sum_{k=1}^{m} \lambda_{k} Y_{P}\left(t_{k}\right)\right\|_{(2 n)}\right) \\
\leqslant & \varlimsup_{n \rightarrow \infty}\left(\frac{1}{2 n} \sum_{k=1}^{m}\left|\lambda_{k}\right|\left\|Y_{P}(t)\right\|_{(2 n)}\right)=\sum_{k=1}^{m}\left|\lambda_{k}\right| \overline{\lim _{n \rightarrow \infty}}\left(\frac{1}{2 n}\left\|Y_{P}(t)\right\|_{(2 n)}\right)<\infty,
\end{aligned}
$$

because $Y_{P}(t)$ has $\Gamma$ distribution, see Proposition 10. Thus, the moments uniquely determine the distribution of the random variable (3.16), and this is what was to be proved. The first two statements of the theorem are proven.

Part 5. Similarly to the beginning of Part 1, we have

$$
\begin{aligned}
\mathrm{E}_{A} \log \varphi_{R_{1,1}}(G) & =\mathrm{E}_{A} \sum_{m=1}^{\infty} \frac{i^{m}}{m} \mu_{0} \sigma_{0}^{2(m-1)} \int_{[0, T]^{m}}\left(\frac{1}{-2 \alpha_{k}} \mathrm{e}^{\alpha_{k} D_{\tau_{0}}(\underline{t})}\right) \mathrm{d} G\left(t_{1}\right) \ldots \mathrm{d} G\left(t_{m}\right) \\
& =\sum_{m=1}^{\infty} \frac{i^{m}}{m} \mu_{0} \sigma_{0}^{2(m-1)} \int_{[0, T]^{m}} \mathrm{E}_{A}\left(\frac{1}{-2 \alpha_{k}} \mathrm{e}^{\alpha_{k} D_{\tau_{0}}(\underline{t})}\right) \mathrm{d} G\left(t_{1}\right) \ldots \mathrm{d} G\left(t_{m}\right) \\
& =\frac{\mu_{0} \lambda}{2 \sigma_{0}^{2}(1-H)} \sum_{m=1}^{\infty} \frac{i^{m}}{m} \sigma_{0}^{2 m} \int_{[0, T]^{m}}\left(1+\frac{D_{\tau_{0}}(\underline{t})}{2 \lambda}\right)^{2(H-1)} \mathrm{d} G\left(t_{1}\right) \ldots \mathrm{d} G\left(t_{m}\right) \\
& =\log \varphi_{Y}(G),
\end{aligned}
$$


for $\operatorname{var}(G)<\sigma_{0}^{-2}$. The change of order of the expectation and the integral, and also the change of order of the expectation and the sum can be easily justified by Fubini's theorem and the fact that the characteristic functionals are only considered for $\operatorname{var}(G)<\sigma_{0}^{-2}$.

We also have to prove, that equation (3.8) holds not only for $\operatorname{var}(G)<\sigma_{0}^{-2}$ but on the whole space $N B V[0, T]$. The exponential of the right-hand side of (3.8) defines a characteristic functional, $\psi(G)$, say, on the whole $N B V[0, T]$ because every positive power of the characteristic functional of any stationary DLG process also will be a characteristic functional (of some stationary DLG process), see Remark 3 . Now, we have two characteristic functionals, $\varphi_{Y}(G)$ and $\psi(G)$, and they both agree with the exponential of the right-hand side of (3.7) for $\operatorname{var}(G)<\sigma_{0}^{-2}$. The situation is the same as it was at (3.13), thus, the conclusion must also be the same, namely that $\varphi_{Y}(G)=\psi(G)$ for all $G \in N B V[0, T]$. Thus (3.8) holds for all $G \in N B V[0, T]$.

The following observation is a consequence of the third statement of Theorem 5 and Remark 3.

Corollary 2. The SDLG process $Y(t)$ is infinitely divisible.

Remark 9. As it appears from the last paragraph of the previous proof, the existence of the SDLG process $Y(t)$ depends eventually on the stationary DLG process being infinitely divisible.

Remark 10. Let $G(t) \stackrel{\circ}{=} u \chi_{\left[t_{1}, T\right]}(t)$, where $u \in \mathbb{R},|u|<\sigma_{0}^{-2}$. Taking function $G(t)$ in Theorem 5 , we have

$$
\log \varphi_{Y(t)}(u)=-\frac{\mu_{0} \lambda}{2 \sigma_{0}^{2}(1-H)} \log \left(1-i u \sigma_{0}^{2}\right)
$$

for $|u|<\sigma_{0}^{-2}$. This is the logarithm of the characteristic function of a $\Gamma$ distribution around zero, and it uniquely determines the characteristic function on the whole line. Thus, we have the result already known, namely that $Y(t) \sim \Gamma\left(\mu_{0} \lambda \sigma_{0}^{-2}(1-H)^{-1} / 2, \sigma_{0}^{2}\right)$, see Corollary 1 .

The following lemma will be needed later:

Lemma 4. Let $m \in \mathbb{N}, t_{1}, \ldots, t_{m} \in[0, T]$. Then the mixture representation

$$
\operatorname{cum}\left(Y\left(t_{1}\right), \ldots, Y\left(t_{m}\right)\right)=\mathrm{E}_{A} \operatorname{cum}\left(R_{1,1}\left(t_{1}\right), \ldots, R_{1,1}\left(t_{m}\right)\right)
$$

holds, where $R_{1,1}(t)$ are the component processes defined above, see the paragraph preceding Remark 7 . The following explicit formula also can be given.

$$
\operatorname{cum}\left(Y\left(t_{1}\right), \ldots, Y\left(t_{m}\right)\right)=\frac{\mu_{0} \lambda \sigma_{0}^{2 m-2}}{2(1-H)} \sum_{\tau \in \operatorname{Perm}(2, \ldots, m)}\left(1+\frac{D_{\tau}(\underline{t})}{2 \lambda}\right)^{2(H-1)}
$$

Proof. The same mixture representation holds for the finite-dimensional, in particular for the $m$-dimensional distributions, as for the distributions of processes on $\mathcal{C}[0, T]$. Thus the same formula holds for the logarithmic $m$ variate characteristic functions, and for the cumulants of order $m$ too. This is

$$
\begin{gathered}
\operatorname{cum}\left(Y\left(t_{1}\right), \ldots, Y\left(t_{m}\right)\right)=\mathrm{E}_{A} \operatorname{cum}\left(R\left(t_{1}\right), \ldots, R\left(t_{m}\right)\right) \\
=\mu_{0} \sigma_{0}^{2 m-2} \sum_{\tau \in \operatorname{Perm}(2, \ldots, m)} \mathrm{E}_{A}\left(\frac{1}{-2 \alpha_{k}} \mathrm{e}^{\alpha_{k} D_{\tau}(\underline{t})}\right)=\frac{\mu_{0} \lambda \sigma_{0}^{2 m-2}}{2(1-H)} \sum_{\tau \in \operatorname{Perm}(2, \ldots, m)}\left(1+\frac{D_{\tau}(\underline{t})}{2 \lambda}\right)^{2(H-1)} .
\end{gathered}
$$

The second equation follows from $(2.22,2.3)$, and Condition 4 and that $\mu=\mu_{0}$. The last equation follows from Condition 3.

Proposition 11. The centered integral process of the SDLG process $Y(t)$ is asymptotically multifractal. Specifically, the following scaling rules hold.

$$
\operatorname{cum}_{m}\left(\int_{0}^{t}(Y(s)-\mathrm{E} Y) \mathrm{d} s\right) \underset{t \rightarrow \infty}{\simeq} k(m) t^{m+2(H-1)},
$$


for $m \geqslant 2$, where $\operatorname{cum}_{m}$ denotes the cumulant of order $m$ of the one-dimensional distribution, $k(m)$, $m \geqslant 2$, are some positive constants not depending on $t$ but depending on $m$, and $\underset{t \rightarrow \infty}{\simeq}$ means asymptotically equivalence as $t \rightarrow \infty$.

Proof. Taking in (3.7) function $G(t)=u t$ with $|u|$ sufficiently small, we have

$$
\begin{gathered}
\operatorname{cum}_{m}\left(\int_{0}^{T} Y(t) \mathrm{d} t\right)=\frac{\mu_{0} \lambda \sigma_{0}^{2 m-2}}{2(1-H)}(m-1) ! \int_{[0, T]^{m}}\left(1+\frac{D_{\tau_{0}}(\underline{t})}{2 \lambda}\right)^{2(H-1)} \mathrm{d} \underline{t} \\
\underset{T \stackrel{\rightarrow}{\rightarrow}}{\simeq} \frac{2^{1-2 H}}{1-H} \mu_{0} \lambda^{3-2 H} \sigma_{0}^{2 m-2}(m-1) ! \int_{[0,1]^{m}} D_{\tau_{0}}(\underline{t})^{2(H-1)} \mathrm{d} \underline{t} T^{m+2(H-1)}=k(m) T^{m+2(H-1)},
\end{gathered}
$$

where the asymptotically equivalence holds for $m \geqslant 2$.

Proposition 12. The SDLG process $Y(t)$ is LRD with parameter $H$.

Proof. Using Lemma 4, the autocovariance function of the stationary process $Y(t)$ is

$$
R_{Y}(t) \stackrel{\circ}{=} \operatorname{cov}(Y(0), Y(t))=\sigma_{0}^{2} \frac{\mu_{0} \lambda}{2(1-H)}\left(1+\frac{|t|}{2 \lambda}\right)^{2(H-1)} \underset{t \rightarrow \infty}{\simeq} \operatorname{const.} t^{2(H-1)},
$$

indicating a hyperbolically decay, and the LRD parameter is $H$.

\subsection{Multifractal limit processes (LISDLG and $\Delta$ LISDLG processes)}

In this subsection we will show that one can go further and make up a subsequent limit process as parameter $\lambda \rightarrow 0$. The new limit process will have interesting properties. Firstly it has the same second order covariance structure as that of the FBM, thus it will be second order self-similar. However, not selfsimilar, but multifractal, in particular exactly multifractal. Furthermore, the increments of the limit process will have a distribution close to $\Gamma$, leastwise for the case of values of $H$ close to 1 . In addition, the bispectrum will also be unusual as it is real-valued, but that is in fact the subject of a later section.

Assume that $\mu_{0}$ depends on $\lambda$ so that $\lim _{\lambda \rightarrow 0}\left(\mu_{0} \lambda^{3-2 H}\right)=c_{0}$, some positive constant. Let us denote the dependence of the SDLG process on $\lambda$, too. Hence $Y(t)=Y_{\lambda}(t)$.

As a consequence of Corollary 1 , we know the expectation of $Y_{\lambda}(t)$, and thus

$$
\mathrm{E} Y_{\lambda}(t)=\frac{\mu_{0} \lambda}{2(1-H)}=\frac{\mu_{0} \lambda^{3-2 H}}{2(1-H)} \lambda^{2(H-1)} \underset{\lambda \rightarrow 0}{\longrightarrow} \infty
$$

because $1 / 2<H<1$, see Condition 1 . Hence, the higher order moments of $Y_{\lambda}(t)$, and also the central moments converge to infinity, therefore the existence of any limit process as $\lambda \rightarrow 0$ is unlikely, even after standardization. However, there may exist a limit process in some Schwartz-distribution sense, which indicates that it is more profitable to deal with the integral or time-aggregated process.

\section{Notation 8.}

$$
J_{\lambda}(t) \stackrel{\circ}{=} \int_{0}^{t}\left(Y_{\lambda}(s)-\mathrm{E} Y_{\lambda}\right) \mathrm{d} s
$$

where the integral is simply assumed to be either pathwise Lebesgue integral or pathwise Riemann integral. Process $J_{\lambda}(t)$ is the integral process of the centralized SDLG process $Y_{\lambda}$, therefore it will be referred to as an Integrated SDLG (ISDLG) process. Its discrete increment process,

$$
\Delta J_{\lambda}(t) \stackrel{\circ}{=} J_{\lambda}(t+1)-J_{\lambda}(t)
$$

$t \in \mathbb{Z}$, will be called a $\Delta$ ISDLG process. 
Remark 11. The ISDLG process $J_{\lambda}(t)$ is infinitely divisible because so is the SDLG process $Y_{\lambda}(t)$, see Corollary 2.

We reassert our latter assumption.

Condition 7. $\lim _{\lambda \rightarrow 0}\left(\mu_{0} \lambda^{3-2 H}\right)=c_{0}>0$.

Because $J_{\lambda}(t)$ is an integral process, it is a.s. continuous, hence it generates a measure on $\mathcal{C}[0, T]$. Now we state that the characteristic functional of the ISDLG process $J_{\lambda}$ converges as $\lambda \rightarrow 0$, around zero, at the very least.

Proposition 13. In a neighborhood of $0 \in N B V[0, T]$ the logarithm of the characteristic functional of the ISDLG process $J_{\lambda}$ converges to a limit, that is to say,

$$
\begin{aligned}
\log \varphi_{J_{\lambda}}(G) & =\sum_{m=1}^{\infty} \frac{i^{m}}{m !} \operatorname{cum}_{m}\left(\int_{0}^{T} J_{\lambda}(t) \mathrm{d} G(t)\right) \\
& \underset{\lambda \rightarrow 0}{\longrightarrow} \frac{2^{1-2 H}}{1-H} \cdot \frac{c_{0}}{\sigma_{0}^{2}} \sum_{m=2}^{\infty} \frac{i^{m}}{m} \sigma_{0}^{2 m} \int_{[0, T]^{m}} D_{\tau_{0}}(\underline{t})^{2(H-1)} \prod_{k=1}^{m}\left(G(T)-G\left(t_{k}\right)\right) \mathrm{d} \underline{t},
\end{aligned}
$$

for

$$
\operatorname{var}(G)<\frac{1}{T \sigma_{0}^{2}},
$$

where $\tau_{0}$ is the identity permutation. (See Notation 5 on $D_{\tau_{0}}(\underline{t})$.)

Proof. Let $G \in N B V[0, T]$, such that (3.19) holds. Let us calculate the cumulants of the random variable

$$
\int_{0}^{T} J_{\lambda}(t) \mathrm{d} G(t) .
$$

The cumulant of order 1 is the expectation, which is zero, because $J_{\lambda}(t)$ is centralized, i.e.

$$
\operatorname{cum}_{1}\left(\int_{0}^{T} J_{\lambda}(t) \mathrm{d} G(t)\right)=\mathrm{E} \int_{0}^{T} J_{\lambda}(t) \mathrm{d} G(t)=0 .
$$

The higher order cumulants are

$$
\begin{aligned}
\operatorname{cum}_{m}\left(\int_{0}^{T} J_{\lambda}(t) \mathrm{d} G(t)\right) & =\operatorname{cum}_{m}\left(\int_{0}^{T} Y_{\lambda}(t)(G(T)-G(t)) \mathrm{d} t\right) \\
& =\int_{[0, T]^{m}} \operatorname{cum}\left(Y_{\lambda}\left(t_{1}\right), \ldots, Y_{\lambda}\left(t_{m}\right)\right) \prod_{k=1}^{m}\left(G(T)-G\left(t_{k}\right)\right) \mathrm{d} \underline{t} \\
& =\frac{\mu_{0} \lambda}{2(1-H)} \sigma_{0}^{2 m-2} \int_{[0, T]^{m}} \sum_{\tau \in \operatorname{Perm}(2, \ldots, m)}\left(1+\frac{D_{\tau}(\underline{t})}{2 \lambda}\right)^{2(H-1)} \prod_{k=1}^{m}\left(G(T)-G\left(t_{k}\right)\right) \mathrm{d} \underline{t} \\
& =\mu_{0} \lambda^{3-2 H} \frac{2^{1-2 H}}{1-H} \sigma_{0}^{2 m-2}(m-1) ! \int_{[0, T]^{m}}^{m}\left(2 \lambda+D_{\tau_{0}}(\underline{t})\right)^{2(H-1)} \prod_{k=1}^{m}\left(G(T)-G\left(t_{k}\right)\right) \mathrm{d} \underline{t} \\
& \underset{\lambda \rightarrow 0}{\longrightarrow} c_{0} \frac{2^{1-2 H}}{1-H} \sigma_{0}^{2 m-2}(m-1) ! \int_{[0, T]^{m}} D_{\tau_{0}}(\underline{t})^{2(H-1)} \prod_{k=1}^{m}\left(G(T)-G\left(t_{k}\right)\right) \mathrm{d} \underline{t},
\end{aligned}
$$


for $m \geqslant 2$. In the second equation the change of the order of the cumulant operator and the integrals can be justified similarly as in (2.26) (see the explanation after (2.26)). In the third equation we used Lemma 4, and the fourth equation arises by performing the proper permutational integral transformations. In reference to the convergence in the last row, we remark that if the $m$-dimensional integral in the last row were finite, then Lebesgue's dominated convergence theorem could be applied, since

$$
\left(2 \lambda+D_{\tau_{0}}(\underline{t})\right)^{2(H-1)} \leqslant D_{\tau_{0}}(\underline{t})^{2(H-1)}
$$

Our backlog, the inequality

$$
\int_{[0, T]^{m}} D_{\tau_{0}}(\underline{t})^{2(H-1)} \prod_{k=1}^{m}\left|G(T)-G\left(t_{k}\right)\right| \mathrm{d} \underline{t}<\infty,
$$

will be a by-product of the following calculation.

$$
\begin{aligned}
\left|\operatorname{cum}_{m}\left(\int_{0}^{T} J_{\lambda}(t) \mathrm{d} G(t)\right)\right| & \leqslant \frac{\mu_{0} \lambda^{3-2 H} 2^{1-2 H} \sigma_{0}^{2 m-2}(m-1) !}{1-H} \int_{[0, T]^{m}} D_{\tau_{0}}(\underline{t})^{2(H-1)} \prod_{k=1}^{m}\left|G(T)-G\left(t_{k}\right)\right| \mathrm{d} \underline{t} \\
& \left.\leqslant \frac{\mu_{0} \lambda^{3-2 H} 2^{1-2 H} \sigma_{0}^{2 m-2}(m-1) !}{1-H} \sup _{t \in[0, T]}|G(T)-G(t)|\right)_{[0, T]^{m}}^{m} D_{\tau_{0}}(\underline{t})^{2(H-1)} \mathrm{d} \underline{t} \\
& \leqslant \text { const. } \frac{\sigma_{0}^{2 m-2}(m-1) ! \operatorname{var}(G)^{m}}{1-H} \int_{[0, T]^{m}}\left|t_{2}-t_{1}\right|^{2(H-1)} \mathrm{d} \underline{t} \\
& =\text { const. } \frac{(m-1) ! T^{2(H-1)}}{\sigma_{0}^{2}(1-H)(2 H-1) H}\left(\sigma_{0}^{2} \operatorname{var}(G) T\right)^{m},
\end{aligned}
$$

where the first inequality is the consequence of the second last row of (3.22) and of (3.23). The second inequality is obvious. The third inequality and the last equality hold because of Condition 7 and the $1 / 2<H<1$ part of Condition 1 .

Using (3.24) we find the series expansion in the first row of (3.18) to be absolutely convergent on the domain given by (3.19). Substituting (3.21) and (3.22) into the first row of (3.18), we get the statement to be proved. In the last step, when changing the order of the infinite sum and the operator $\lim _{\lambda \rightarrow 0}$, we could apply Lebesgue's theorem because of (3.24).

Lemma 5. The set of measures on $\mathcal{C}[0, T]$ induced by the ISDLG processes $J_{\lambda}, \lambda>0$, is tight.

Proof. Let $\lambda>0$ be arbitrary. The ISDLG process $J_{\lambda}(t)$ has stationary increments, therefore

$$
\mathrm{E}\left(J_{\lambda}(s+t)-J_{\lambda}(s)\right)^{2}=\mathrm{E} J_{\lambda}(t)^{2}=\operatorname{cum}_{2}\left(J_{\lambda}(t)\right) .
$$

We will refer to the proof of Proposition 13. It is easy to check that the equation part of (3.22), i.e. (3.22) except for the last row, holds for all $G \in N B V[0, T]$. Performing the substitution $G(s)=\chi_{[t, T]}(s)$ in the second last row of $(3.22)$ for $m=2$, we have

$$
\begin{gathered}
\quad \operatorname{cum}_{2}\left(J_{\lambda}(t)\right)=\mu_{0} \lambda^{3-2 H} \frac{2^{1-2 H}}{1-H} \sigma_{0}^{2} \int_{[0, t]^{2}}\left(2 \lambda+2\left|s_{2}-s_{1}\right|\right)^{2(H-1)} \mathrm{d} s_{2} \mathrm{~d} s_{1} \\
\leqslant \text { const. } \frac{\sigma_{0}^{2}}{1-H} \int_{[0, t]^{2}}\left|s_{2}-s_{1}\right|^{2(H-1)} \mathrm{d} s_{2} \mathrm{~d} s_{1}=\text { const. } \frac{\sigma_{0}^{2}}{(2 H-1) H(1-H)} t^{2 H},
\end{gathered}
$$

where the inequality follows because of the $1 / 2<H<1$ part of Condition 1. Equations (3.25) and (3.26) together show that Kolmogorov's tightness condition is satisfied. 
Now in the main theorem in this subsection, we state the convergence as $\lambda \rightarrow 0$, of the family of ISDLG processes $J_{\lambda}(t), \lambda>0$, and give a series expansion for the logarithmic characteristic functional of the limit process.

Theorem 6. There exists an a.s. continuous process $J$ such that

$$
J_{\lambda} \underset{\lambda \rightarrow 0}{\stackrel{w}{\longrightarrow} J} \quad \text { in } \mathcal{C}[0, T]
$$

In a neighborhood of $0 \in N B V[0, T]$ the logarithmic characteristic functional of the limit process $J$ has the series expansion

$$
\log \varphi_{J}(G)=\frac{2^{1-2 H}}{1-H} \cdot \frac{c_{0}}{\sigma_{0}^{2}} \sum_{m=2}^{\infty} \frac{i^{m}}{m} \sigma_{0}^{2 m} \int_{[0, T]^{m}} D_{\tau_{0}}(\underline{t})^{2(H-1)} \prod_{k=1}^{m}\left(G(T)-G\left(t_{k}\right)\right) \mathrm{d} \underline{t},
$$

for

$$
\operatorname{var}(G)<\frac{1}{\sigma_{0}^{2} T}
$$

where $\tau_{0}$ is the identity permutation.

Because process $J(t)$ is the Limit of ISDLG processes, it will be called a LISDLG process.

Proof. The reader can check that the proof of Theorem 5 as far as Part 4 can be applied to the present case too. That is to say, in the same way, we already have the tightness and the convergence of the characteristic functionals around zero, see Lemma 5 and Proposition 13, respectively. The only point, that we have to amend is in Part 4. We adapt the notations of the proof mentioned and continue it from the beginning of Part 4.

We have to prove that the moments of the random variable

$$
\sum_{k=1}^{m} \lambda_{k} J_{P}\left(t_{k}\right)
$$

uniquely determine its distribution, and the proof is complete. Let us consider the expression in the moment condition. We can estimate it as follows.

$$
\varlimsup_{n \rightarrow \infty}\left(\frac{1}{2 n}\left(\mathrm{E}\left|\sum_{k=1}^{m} \lambda_{k} J_{P}\left(t_{k}\right)\right|^{2 n}\right)^{\frac{1}{2 n}}\right) \leqslant \sum_{k=1}^{m}\left|\lambda_{k}\right| \max _{1 \leqslant j \leqslant m} \varlimsup_{n \rightarrow \infty}\left(\frac{1}{2 n}\left\|J_{P}\left(t_{j}\right)\right\|_{(2 n)}\right) .
$$

Thus what we have to prove is

$$
\varlimsup_{n \rightarrow \infty}\left(\frac{1}{2 n}\left\|J_{P}(t)\right\|_{(2 n)}\right)<\infty
$$

for all $t \in[0, T]$.

For this end we use the expression of moments via cumulants since the cumulants are more tractable now than the moments. At first let us show how to express the joint central moments of the SDLG process $Y(t)$ by its joint cumulants. The general formula we apply is formula (1.9) in [40]. Thus we have

$$
\mathrm{E} \prod_{j=1}^{m}\left(Y\left(t_{j}\right)-\mathrm{E} Y(t)\right)=\sum_{\mathcal{L} \in \operatorname{Part}_{(2 \leqslant)}(1, \ldots, m)} \prod_{K \in \mathcal{L}} \operatorname{cum}\left(Y\left(\underline{t}_{(K)}\right)\right) .
$$

Explanation of the notations: $\operatorname{Part}(1, \ldots, m)$ is the set of all partitions of the set $\{1, \ldots, m\}$ and the set of partitions the elements of which have at least two elements is denoted by $\operatorname{Part}_{(2 \leqslant)}(1, \ldots, m)$, i.e.

$$
\operatorname{Part}_{(2 \leqslant)}(1, \ldots, m) \stackrel{\circ}{=}\{\mathcal{L} \in \operatorname{Part}(1, \ldots, m): \forall K \in \mathcal{L}:|K| \geqslant 2\}
$$


Moreover, $Y\left(\underline{t}_{(K)}\right)=\left(Y\left(t_{i_{1}}\right), \ldots, Y\left(t_{i_{k}}\right)\right)$, if $K=\left\{i_{1}, \ldots, i_{k}\right\}$.

Replacing the cumulants in (3.30) by their expressions in (3.17), we have

$$
\begin{aligned}
& \mathrm{E} \prod_{j=1}^{m}\left(Y\left(t_{j}\right)-\mathrm{E} Y(t)\right)=\sum_{\mathcal{L} \in \operatorname{Part}_{(2 \leqslant)}(1, \ldots, m)} \prod_{K \in \mathcal{L}}\left(\frac{\mu_{0} \lambda^{3-2 H}}{2(1-H)} \sigma_{0}^{2|K|-2} \sum_{\tau \in \operatorname{Perm}(K \backslash\{\min (K)\})}\left(2 \lambda+D_{\tau}\left(\underline{t}_{(K)}\right)\right)^{2(H-1)}\right) \\
& =\sum_{\mathcal{L} \in \operatorname{Part}_{(2 \leqslant)}(1, \ldots, m)}\left(\left(\frac{\mu_{0} \lambda^{3-2 H}}{2(1-H) \sigma_{0}^{2}}\right)^{|\mathcal{L}|} \sigma_{0}^{2 m} \prod_{K \in \mathcal{L}} \sum_{\tau \in \operatorname{Perm}(K \backslash\{\min (K)\})}\left(2 \lambda+D_{\tau}\left(\underline{t}_{(K)}\right)\right)^{2(H-1)}\right) .
\end{aligned}
$$

Hence,

$$
\begin{aligned}
& \mathrm{E}\left(J_{\lambda}(t)\right)^{m}=\int_{[0, t]^{m}} \mathrm{E} \prod_{j=1}^{m}\left(Y\left(s_{j}\right)-\mathrm{E} Y\right) \mathrm{d} \underline{s} \\
& =\sum_{\mathcal{L} \in \operatorname{Part}_{(2 \leqslant)}(1, \ldots, m)}\left[\left(\frac{\mu_{0} \lambda^{3-2 H}}{\sigma_{0}^{2}} \cdot \frac{1}{2(1-H)}\right)^{|\mathcal{L}|} \sigma_{0}^{2 m}\right. \\
& \left.\quad \times \prod_{K \in \mathcal{L}}\left((|K|-1) ! \int_{[0, t]^{|K|}} \sum_{\tau \in \operatorname{Perm}(K \backslash\{\min (K)\})}\left(2 \lambda+D_{\tau_{0}}\left(\underline{s}_{(K)}\right)\right)^{2(H-1)} \mathrm{d} \underline{s}_{(K)}\right)\right] \\
& \underset{\lambda \rightarrow 0}{\longrightarrow}\left(t \sigma_{0}^{2}\right)^{m} \sum_{\mathcal{L} \in \operatorname{Part}(2 \leqslant)(1, \ldots, m)}\left[\left(\frac{1}{2(1-H)} \cdot \frac{c_{0}}{\sigma_{0}^{2}} t^{2(H-1)}\right)^{|\mathcal{L}|} \prod_{K \in \mathcal{L}}\left((|K|-1) ! \int_{[0,1]^{|K|}} D_{\tau_{0}}\left(\underline{s}_{(K)}\right)^{2(H-1)} \mathrm{d} \underline{s}_{(K)}\right)\right]
\end{aligned}
$$

where the change of order of the integral and the limes as $\lambda \rightarrow 0$ is justifiable because of the inequality

$$
\left(2 \lambda+D_{\tau_{0}}(\underline{s})\right)^{2(H-1)} \leqslant D_{\tau_{0}}(\underline{s})^{2(H-1)} .
$$

After these preparations we can return to the actual proof of inequality (3.29).

$$
\begin{aligned}
& \varlimsup_{n \rightarrow \infty}\left(\frac{1}{2 n}\left\|J_{P}(t)\right\|_{(2 n)}\right)=\varlimsup_{n \rightarrow \infty}\left\{\frac{1}{2 n}\left[\left(t \sigma_{0}^{2}\right)^{2 n} \sum_{\mathcal{L} \in \operatorname{Part}_{(2 \leqslant)}(1, \ldots, 2 n)}\left(\frac{1}{2(1-H)} \cdot \frac{c_{0}}{\sigma_{0}^{2}} t^{2(H-1)}\right)^{|\mathcal{L}|}\right]\right. \\
& \left.\times \prod_{K \in \mathcal{L}}\left((|K|-1) ! \int_{[0,1]^{|K|}} D_{\tau_{0}}\left(\underline{s}_{(K)}\right)^{2(H-1)} \mathrm{d} \underline{s}_{(K)}\right)^{\frac{1}{2 n}}\right\} \\
& \leqslant t \sigma_{0}^{2} \varlimsup_{n \rightarrow \infty}\left\{\frac{1}{2 n}\left[\sum_{\mathcal{L} \in \operatorname{Part}(1, \ldots, 2 n)}\left(\frac{1}{2(1-H)} \cdot \frac{c_{0}}{\sigma_{0}^{2}} t^{2(H-1)}\right)^{|\mathcal{L}|} \prod_{K \in \mathcal{L}}\left((|K|-1) ! \frac{1}{(2 H-1) H}\right)\right]^{\frac{1}{2 n}}\right\} \\
& \leqslant t \sigma_{0}^{2} \max \left\{1, \frac{1}{(2 H-1)(1-H) 2 H} \cdot \frac{c_{0}}{\sigma_{0}^{2}} t^{2(H-1)}\right\} \varlimsup_{n \rightarrow \infty}\left\{\frac{1}{2 n}\left[\sum_{\mathcal{L} \in \operatorname{Part}(1, \ldots, 2 n)} \prod_{K \in \mathcal{L}}(|K|-1) !\right]^{\frac{1}{2 n}}\right\} .
\end{aligned}
$$


At the same time, if we take $2 n$ identical copies of a $\Gamma(1,1)$ distributed random variable, i.e. $\xi_{1}=\ldots$ $=\xi_{2 n} \sim \Gamma(1,1)$, and express their joint moment by the joint cumulants, we get the very identity

$$
\begin{aligned}
& (2 n) !=\mathrm{E} \xi_{1}^{2 n}=\mathrm{E} \prod_{j=1}^{2 n} \xi_{j}=\sum_{\mathcal{L} \in \operatorname{Part}(1, \ldots, 2 n)} \prod_{K \in \mathcal{L}} \operatorname{cum}\left(\underline{\xi}_{(K)}\right) \\
= & \sum_{\mathcal{L} \in \operatorname{Part}(1, \ldots, 2 n)} \prod_{K \in \mathcal{L}} \operatorname{cum}_{|K|}\left(\xi_{1}\right)=\sum_{\mathcal{L} \in \operatorname{Part}(1, \ldots, 2 n)} \prod_{K \in \mathcal{L}}(|K|-1) !,
\end{aligned}
$$

where in the third equation the general expression of the moments via cumulants is used again, see formula (1.9) in [40]. Substituting the result of (3.32) into (3.31), we obtain the inequalities

$$
\varlimsup_{n \rightarrow \infty}\left(\frac{1}{2 n}\left\|J_{P}(t)\right\|_{(2 n)}\right) \leqslant \text { const. } \varlimsup_{n \rightarrow \infty}\left(\frac{1}{2 n}((2 n) !)^{\frac{1}{2 n}}\right) \leqslant \text { const. } \varlimsup_{n \rightarrow \infty}\left(\frac{1}{2 n} \cdot \frac{1}{2 n}(1+2+\cdots+2 n)\right)<\infty .
$$

We arrived at (3.29), which was to be proved.

Since the ISDLG process $J_{\lambda}$ is infinitely divisible (see Rem. 11), and the weak limit of infinitely divisible processes also has this property, we have the following corollary:

Corollary 3. The LISDLG process $J(t)$ is infinitely divisible.

The ISDLG processes $J_{\lambda}, \lambda>0$, being integral processes of stationary processes, have stationary increments, hence the following remark is true:

Remark 12. The LISDLG process $J(t)$ has stationary increments.

After the SDLG process $Y_{\lambda}(t)$, we defined the ISDLG process $J_{\lambda}(t)$, which is asymptotically multifractal, see Proposition 11. Taking the limit as $\lambda \rightarrow 0$, we have arrived at our object, the limit process $J(t)$. Our motive to do so was that the LISDLG process $J(t)$ is already exactly multifractal.

Theorem 7. The LISDLG process $J(t)$ has the following cumulants

$$
\operatorname{cum}_{1}(J(t))=0
$$

and

$$
\begin{aligned}
\operatorname{cum}_{m}(J(t)) & =(m-1) ! \frac{2^{1-2 H}}{1-H} c_{0} \sigma_{0}^{2(m-1)} \int_{[0,1]^{m}} D_{\tau_{0}}(\underline{s})^{2(H-1)} \mathrm{d} \underline{s} t^{m+2(H-1)} \\
& =k_{1}(m) t^{m+2(H-1)}
\end{aligned}
$$

for $m \geqslant 2$. (See also Prop. 11 for comparison.) Therefore, the LISDLG process $J(t), t \in \mathbb{R}$, is multifractal.

Proof. Let us consider the function $G(s)=u \chi_{[t, T]}(s), 0 \leqslant s \leqslant T$, where $0 \leqslant|u|<\sigma_{0}^{-2} T^{-1}$, and substitute it into (3.27). The replacement is legitimate since $\operatorname{var}(G)=|u|<\sigma_{0}^{-2} T^{-1}$, and for the cumulant generator function $\log \varphi_{J(t)}(u)$ we have

$$
\begin{aligned}
\log \varphi_{J(t)}(u) & =\log \varphi_{J}(G) \\
& =\frac{2^{1-2 H}}{1-H} \cdot \frac{c_{0}}{\sigma_{0}^{2}} \sum_{m=2}^{\infty} \frac{i^{m}}{m} \sigma_{0}^{2 m} \int_{[0, T]^{m}} D_{\tau_{0}}(\underline{s})^{2(H-1)} \prod_{k=1}^{m}\left(G(T)-G\left(s_{k}\right)\right) \mathrm{d} \underline{s} \\
& =\frac{2^{1-2 H}}{1-H} \cdot \frac{c_{0}}{\sigma_{0}^{2}} \sum_{m=2}^{\infty} \frac{(i u)^{m}}{m} \sigma_{0}^{2 m} \int_{[0, t]^{m}} D_{\tau_{0}}(\underline{s})^{2(H-1)} \mathrm{d} \underline{s} \\
& =\sum_{m=2}^{\infty} \frac{(i u)^{m}}{m !}(m-1) ! \frac{2^{1-2 H}}{1-H} \cdot \frac{c_{0}}{\sigma_{0}^{2}} \sigma_{0}^{2 m} \int_{[0, t]^{m}} D_{\tau_{0}}(\underline{s})^{2(H-1)} \mathrm{d} \underline{s},
\end{aligned}
$$


that is,

$$
\operatorname{cum}_{1}(J(t))=0
$$

and

$$
\begin{aligned}
\operatorname{cum}_{m}(J(t)) & =(m-1) ! \frac{2^{1-2 H}}{1-H} \cdot \frac{c_{0}}{\sigma_{0}^{2}} \sigma_{0}^{2 m} \int_{[0, t]^{m}} D_{\tau_{0}}(\underline{s})^{2(H-1)} \mathrm{d} \underline{s} \\
& =(m-1) ! \frac{2^{1-2 H}}{1-H} \cdot \frac{c_{0}}{\sigma_{0}^{2}} \sigma_{0}^{2 m} \int_{[0,1]^{m}} D_{\tau_{0}}(\underline{s})^{2(H-1)} \mathrm{d} \underline{s} t^{m+2(H-1)},
\end{aligned}
$$

for $m \geqslant 2$.

Corollary 4. The discrete increment process $\Delta J(t) \stackrel{\circ}{=}(t+1)-J(t), t \in \mathbb{Z}$, is multifractal. We will call it a $\triangle L I S D L G$ process.

Proof. Indeed the aggregated increments $\Delta J(k)^{(n)}$, i.e.

$$
\Delta J(k)^{(n)}=\frac{1}{n} \sum_{j=0}^{n-1} \Delta J(k n-j)=\frac{1}{n}(J(k n+1)-J((k-1) n+1)) \stackrel{d}{=} \frac{1}{n} J(n),
$$

have cumulants of order $m$

$$
\operatorname{cum}_{m}\left(\Delta J(k)^{(n)}\right)=(m-1) ! \frac{2^{1-2 H}}{1-H} c_{0} \sigma_{0}^{2(m-1)} \int_{[0,1]^{m}} D_{\tau_{0}}(\underline{s})^{2(H-1)} \mathrm{d} \underline{s} n^{2(H-1)}=k_{1}(m) n^{2(H-1)}
$$

as it follows from Theorem 7. The exponent of $n$ is $2 H-2=m\left(\frac{2 H-2+m}{m}-1\right)$, i.e. the Hurst exponent depends on $m$ :

$$
H(m)=\frac{2 H-2+m}{m}=\frac{2 H-2}{m}+1
$$

The following is also an outcome of Theorem 7:

Corollary 5. The LISDLG process $J(t)$ has the same covariance structure as the FBM. That is,

$$
\operatorname{cov}\left(J\left(t_{1}\right), J\left(t_{2}\right)\right)=\text { const. }\left(t_{1}^{2 H}+t_{2}^{2 H}-\left|t_{2}-t_{1}\right|^{2 H}\right)
$$

$t_{1}, t_{2}>0$, where the constant does not depend on time. Moreover, the $\Delta L I S D L G$ process $\Delta J(t)$ is LRD with parameter $H$.

Proof. As the LISDLG process $J(t)$ has stationary increments, we have

$$
\operatorname{cov}\left(J\left(t_{1}\right), J\left(t_{2}\right)\right)=\frac{1}{2}\left(\mathrm{D}^{2}\left(J\left(t_{1}\right)\right)+\mathrm{D}^{2}\left(J\left(t_{2}\right)\right)-\mathrm{D}^{2}\left(J\left(t_{2}-t_{1}\right)\right)\right),
$$

where the operator $\mathrm{D}^{2}$ denotes the variance. The variance is the second order cumulant, therefore by substituting into (3.35) the expression for the second order cumulant from Theorem 7 , we have

$$
\operatorname{cov}\left(J\left(t_{1}\right), J\left(t_{2}\right)\right)=\text { const. }\left(t_{1}^{2 H}+t_{2}^{2 H}-\left|t_{2}-t_{1}\right|^{2 H}\right)
$$

thus we have proved the first part of the corollary.

LRD is a property depending only on the second order covariance structure. On the other hand, the $H$-FGN, the increment process of the FBM with parameter $H$, is LRD with parameter $H$. In this manner the second part is proved too. 
By the first statement of Corollary 5 , the second order cumulant structure of the LISDLG process $J(t)$ is the same as that of the FBM. The question is how to proceed, what is the general formula for the higher order joint cumulants? The question relative to a closed formula is open. However, on the basis of the foregoing it is not hard to arrive at the following form:

Proposition 14. The $2 \leqslant m$-th order joint cumulants of the LISDLG process $J(t)$ are

$$
\operatorname{cum}\left(J\left(t_{1}\right), \ldots, J\left(t_{m}\right)\right)=\text { const. } \sigma_{0}^{2 m}(m-1) ! \underset{\underline{t}}{\operatorname{sym}}\left(\int_{0}^{\underline{t}} D_{\tau_{0}}(\underline{s})^{2(H-1)} \mathrm{d} \underline{s}\right)
$$

for $0<t_{1}, \ldots, t_{m}$, where the operator sym is the symmetrization operator, i.e. symmetrization for all

permutations of the components of the vector $\underline{t}=\left(t_{1}, \ldots, t_{m}\right)$. Moreover, the constant depends neither on time, nor on $m$.

Proof. The proof is similar to that of Theorem 7. Let us consider the function

$$
G(s) \doteq \sum_{i=1}^{m} u_{i} \chi_{\left[t_{i}, T\right]}(s)
$$

$0 \leq s \leq T$, where

$$
\sum_{i=1}^{m}\left|u_{i}\right|<\sigma_{0}^{-2} T^{-1}
$$

and substitute it into (3.27). The replacement is legitimate, since

$$
\operatorname{var}(G)=\sum_{i=1}^{m}\left|u_{i}\right|<\sigma_{0}^{-2} T^{-1}
$$

and for the cumulant function $\log \varphi_{J\left(t_{1}\right), \ldots, J\left(t_{m}\right)}\left(u_{1}, \ldots, u_{m}\right)$ we have

$$
\begin{aligned}
\log \varphi_{J\left(t_{1}\right), \ldots, J\left(t_{m}\right)}(\underline{u}) & =\log \varphi_{J}(G) \\
& =\frac{2^{1-2 H}}{1-H} \cdot \frac{c_{0}}{\sigma_{0}^{2}} \sum_{k=2}^{\infty} \frac{i^{k}}{k} \sigma_{0}^{2 k} \int_{[0, T]^{k}} D_{\tau_{0}}(\underline{s})^{2(H-1)} \prod_{j=1}^{k}\left(G(T)-G\left(s_{j}\right)\right) \mathrm{d} \underline{s} \\
& =\frac{2^{1-2 H}}{1-H} \cdot \frac{c_{0}}{\sigma_{0}^{2}} \sum_{k=2}^{\infty} \frac{i^{k}}{k} \sigma_{0}^{2 k} \sum_{i_{1}, \ldots, i_{k}=1}^{m} u_{i_{1}} \cdots u_{i_{k}} \int_{0}^{t_{i_{1}}} \cdots \int_{0}^{t_{i_{k}}} D_{\tau_{0}}(\underline{s})^{2(H-1)} \mathrm{d} \underline{s} \\
& =\sum_{k=2}^{\infty} \frac{1}{k !} \sum_{i_{1}, \ldots, i_{k}=1}^{m} \frac{2^{1-2 H}}{1-H} \cdot \frac{c_{0}}{\sigma_{0}^{2}} \sigma_{0}^{2 k}(k-1) !\left(i u_{1}\right) \cdots\left(i u_{k}\right) \int_{0}^{t_{i_{1}}} \cdots \int_{0}^{t_{i_{k}}} D_{\tau_{0}}(\underline{s})^{2(H-1)} \mathrm{d} \underline{s} .
\end{aligned}
$$

Thus we have

$$
\operatorname{cum}\left(J\left(t_{1}\right), \ldots, J\left(t_{m}\right)\right)=\frac{2^{1-2 H}}{1-H} \cdot \frac{c_{0}}{\sigma_{0}^{2}} \sigma_{0}^{2 m}(m-1) ! \operatorname{sym}_{\underline{t}}\left(\int_{0}^{\underline{t}} D_{\tau_{0}}(\underline{s})^{2(H-1)} \mathrm{d} \underline{s}\right),
$$

for $m \geq 2$. 
Unfortunately, equation (3.36) is not a closed formula. However, there would be a closed formula for the joint cumulants of the derivative process $J^{\prime}(t)=\frac{\mathrm{d}}{\mathrm{d} t} J(t)$, if the latter process existed, viz.

$$
\begin{aligned}
\operatorname{cum}\left(J^{\prime}\left(t_{1}\right), \ldots, J^{\prime}\left(t_{m}\right)\right) & =\frac{\mathrm{d}^{m}}{\mathrm{~d} t_{1} \cdots \mathrm{d} t_{m}} \operatorname{cum}\left(J\left(t_{1}\right), \ldots, J\left(t_{m}\right)\right) \\
& =\operatorname{const.} \sigma_{0}^{2 m}(m-1) ! \operatorname{sym}_{\underline{t}}\left(D_{\tau_{0}}(\underline{t})^{2(H-1)}\right) \\
& =\operatorname{const.} \sigma_{0}^{2 m} \sum_{\tau \in \operatorname{Perm}(2, \ldots, m)} D_{\tau}(\underline{t})^{2(H-1)} \\
& =\lim _{\lambda \rightarrow 0} \operatorname{cum}\left(Y_{\lambda}\left(t_{1}\right), \ldots, Y_{\lambda}\left(t_{m}\right)\right)
\end{aligned}
$$

Because of stationarity, for $m=2$ and $m=3$ the latter means that

$$
\operatorname{cum}\left(J^{\prime}(0), J^{\prime}(t)\right)=\text { const }_{2}|t|^{2(H-1)}=\operatorname{const}_{2}^{\prime}\left(\frac{\mathrm{d}}{\mathrm{d} t}\right)^{2}|t|^{2 H},
$$

for $t \neq 0$, and

$$
\begin{aligned}
\operatorname{cum}\left(J^{\prime}(0), J^{\prime}\left(t_{1}\right), J^{\prime}\left(t_{2}\right)\right) & =\operatorname{const}_{3}\left(\left|t_{1}\right|+\left|t_{2}-t_{1}\right|+\left|t_{2}\right|\right)^{2(H-1)} \\
& =\operatorname{const}_{3}\left(2 \operatorname{diam}\left(0, t_{1}, t_{2}\right)\right)^{2(H-1)} \\
& =\left.\operatorname{const}_{3}^{\prime}\left(\frac{\mathrm{d}}{\mathrm{d} t}\right)^{2}|t|^{2 H}\right|_{t=\operatorname{diam}\left(0, t_{1}, t_{2}\right)}
\end{aligned}
$$

for $t_{1} \neq 0, t_{2} \neq 0, t_{1} \neq t_{2}$, respectively. $\operatorname{In}(3.39) \operatorname{diam}(a, b, c) \stackrel{\circ}{=} \max (a, b, c)-\min (a, b, c)$ is the diameter, and the various constants are all positive.

Now, the discrete time counterpart of $J^{\prime}$, the $\Delta$ LISDLG process $\Delta J(t), t \in \mathbb{Z}$, already exists and it is a stationary process. As they are easy to obtain, the formulas corresponding to (3.38) and (3.39) in discrete time are

$$
\operatorname{cum}(\Delta J(0), \Delta J(t))=\widetilde{\mathrm{const}_{2}}\left(|t+1|^{2 H}-2|t|^{2 H}+|t-1|^{2 H}\right)=\widetilde{\operatorname{const}_{2}} \Delta_{\frac{1}{2}}^{2}|t|^{2 H},
$$

for $t \neq 0$, where $\Delta_{\frac{1}{2}} f(x)=f\left(x+\frac{1}{2}\right)-f\left(x-\frac{1}{2}\right)$ is the central difference operator and $\Delta_{\frac{1}{2}}^{2}$ is its square, and

$$
\begin{aligned}
\operatorname{cum}\left(\Delta J(0), \Delta J\left(t_{1}\right), \Delta J\left(t_{2}\right)\right) & =\left.\widetilde{\operatorname{const}_{3}}\left(|t+1|^{2 H}-2|t|^{2 H}+|t-1|^{2 H}\right)\right|_{t=\operatorname{diam}\left(0, t_{1}, t_{2}\right)} \\
& =\left.\widetilde{\operatorname{const}_{3}} \Delta_{\frac{1}{2}}^{2}|t|^{2 H}\right|_{t=\operatorname{diam}\left(0, t_{1}, t_{2}\right)},
\end{aligned}
$$

for $t_{1} \neq 0, t_{2} \neq 0, t_{1} \neq t_{2}$, respectively.

Remark 13. It is interesting that in formula (3.40) the cumulant does not depend on the middle of the points $0, t_{1}, t_{2}$, it only depends on the diameter of the set of the three points. This property is stronger than the following one.

$$
\operatorname{cum}\left(\Delta J(0), \Delta J\left(t_{1}\right), \Delta J\left(t_{2}\right)\right)=\operatorname{cum}\left(\Delta J(0), \Delta J\left(t_{2}-t_{1}\right), \Delta J\left(t_{2}\right)\right),
$$

for all $t_{1}, t_{2} \in \mathbb{Z}$. The latter is equivalent to the fact that the bispectrum of the $\Delta$ LISDLG process $\Delta J(t)$ is real-valued, see Remark 21.

Now we state two important properties of the finite dimensional distributions of the LISDLG process. 
Theorem 8. The LISDLG process $J(t)$ is $c(p)=p^{\frac{1}{2(H-1)}}$-dilative stable (see Def. 3), i.e. for every $p>0$, $k \in \mathbb{N}$ and $t_{1}, \ldots, t_{k} \geqslant 0$, the characteristic function $\varphi$ of the $k$-dimensional distribution satisfies

$$
\left(\varphi_{\left(J\left(t_{1}\right), \ldots, J\left(t_{k}\right)\right)}\left(u_{1}, \ldots, u_{k}\right)\right)^{p}=\varphi_{p^{-\frac{1}{2(H-1)}}}\left(J\left(p^{\frac{1}{2(H-1)}} t_{1}\right), \ldots, J\left(p^{\frac{1}{2(H-1)}} t_{k}\right)\right)\left(u_{1}, \ldots, u_{k}\right),
$$

i.e.

$$
\left(\varphi_{\left(J\left(t_{1}\right), \ldots, J\left(t_{k}\right)\right)}\left(u_{1}, \ldots, u_{k}\right)\right)^{c^{2(H-1)}}=\varphi_{c^{-1}\left(J\left(c t_{1}\right), \ldots, J\left(c t_{k}\right)\right)}\left(u_{1}, \ldots, u_{k}\right)
$$

for all $u_{1}, \ldots, u_{k} \in \mathbb{R}$.

Proof. Let $T \geq \max \left(t_{1}, \ldots, t_{k}\right)$ and let us consider the function

$$
G(s) \doteq \sum_{j=1}^{k} u_{j} \chi_{\left[t_{j}, T\right]}(s)
$$

$0 \leq s \leq T$, where

$$
\sum_{j=1}^{k}\left|u_{j}\right|<\sigma_{0}^{-2} T^{-1}
$$

and substitute it into (3.27). The replacement is legitimate, since

$$
\operatorname{var}(G)=\sum_{j=1}^{k}\left|u_{j}\right|<\sigma_{0}^{-2} T^{-1}
$$

and for the cumulant function $\log \varphi_{J\left(t_{1}\right), \ldots, J\left(t_{k}\right)}\left(u_{1}, \ldots, u_{k}\right)$ we have

$$
\begin{aligned}
\log \varphi_{J\left(t_{1}\right), \ldots, J\left(t_{k}\right)}(\underline{u}) & =\log \varphi_{J}(G) \\
& =\frac{2^{1-2 H}}{1-H} \cdot \frac{c_{0}}{\sigma_{0}^{2}} \sum_{\ell=2}^{\infty} \frac{i^{\ell}}{\ell} \sigma_{0}^{2 \ell} \int_{[0, T]^{\ell}} D_{\tau_{0}}(\underline{s})^{2(H-1)} \prod_{j=1}^{\ell}\left(G(T)-G\left(s_{j}\right)\right) \mathrm{d} \underline{s} \\
& =\frac{2^{1-2 H}}{1-H} \cdot \frac{c_{0}}{\sigma_{0}^{2}} \sum_{\ell=2}^{\infty} \frac{i^{\ell}}{\ell} \sigma_{0}^{2 \ell} \sum_{i_{1}, \ldots, i_{\ell}=1}^{k} u_{i_{1}} \cdots u_{i_{\ell}} \int_{0}^{t_{i_{1}}} \cdots \int_{0}^{t_{i_{\ell}}} D_{\tau_{0}}(\underline{s})^{2(H-1)} \mathrm{d} \underline{s}
\end{aligned}
$$

for $\underline{u} \in \mathbb{R}^{k}: \sum_{j=1}^{k}\left|u_{j}\right|<\sigma_{0}^{-2} T^{-1}$. From (3.43) by a change of variable in the integral we have (3.42) for $\underline{u}$ $\in \mathbb{R}^{k}: \sum_{j=1}^{k}\left|u_{j}\right|<\sigma_{0}^{-2} T^{-1}$

Since the distribution of $\left(J\left(t_{1}\right), \ldots, J\left(t_{k}\right)\right)$ is infinitely divisible (see Cor. 3 ), the left hand side of (3.42) is a characteristic function. On the other hand, the characteristic function on the right hand side of (3.42) is uniquely determined by its values in any neighborhood of $\underline{0} \in \mathbb{R}^{k}$. This last fact follows easily if one considers the proof of Theorem 6 (with $J_{P}=J$ ) from formula (3.28). Thus we have proved (3.42) for all $\underline{u} \in \mathbb{R}^{k}$.

Corollary 6. The finite-dimensional distributions of the LISDLG process $J(t)$ are infinitely divisible.

Proof. It is a direct consequence of Theorem 8 or either of Corollary 3.

Hereafter several facts follow about the one-dimensional distributions of the increments of the LISDLG process $J(t)$. Because of the stationary increments, it suffices to examine the distribution of $J(t)$ itself. 
Proposition 15. The distribution of the random variable $J(t)$ is uniquely determined by its cumulants given by (3.33). For each $t>0$ the logarithm of the characteristic function of $J(t)$ has the series expansion

$$
\log \varphi_{J(t)}(u)=\sum_{m=2}^{\infty}\left(\frac{(i u)^{m}}{m} \cdot \frac{2^{1-2 H}}{1-H} c_{0} \sigma_{0}^{2(m-1)} \int_{[0,1]^{m}} D_{\tau_{0}}(\underline{s})^{2(H-1)} \mathrm{d} \underline{s} t^{m+2(H-1)}\right),
$$

in the neighborhood $\left\{u:|u|<\sigma_{0}^{-2} t^{-1}\right\}$ of zero.

Proof. The cumulants and the series expansion have been calculated in the proof of Theorem 7 . The uniqueness has also been proved, see the proof of Theorem 6 .

The next two propositions are special cases of Corollary 6 and of Theorem 8 .

Proposition 16. The distribution of the random variable $J(t)$ is infinitely divisible.

Proposition 17. For each $t \in \mathbb{R}_{+}$the characteristic function of the random variable $J(t) / t$ has the following property, called one-dimensional dilative stability. (See Def. 3, Th. 8 and also Sect. 4.1 for an application.)

$$
\varphi_{\frac{J(t)}{t}}(u)=\left(\varphi_{J(1)}(u)\right)^{t^{2(H-1)}},
$$

for all $u \in \mathbb{R}$.

Actually the LISDLG process is derived from the SDLG process, which has $\Gamma$ one-dimensional distribution. So, one can expect that the one-dimensional distribution of the LISDLG process cannot be far from the shifted $\Gamma$ distribution. Now, we examine if it is so. If $H \rightarrow 1$, then the $m$-fold integral in (3.33) converges to one. Therefore if one sets the additional condition

$$
\frac{c_{0}}{2(1-H)} \underset{H \rightarrow 1}{\longrightarrow} c_{1},
$$

where $c_{1}$ is a positive constant, then

$$
\operatorname{cum}_{m}(J(t)) \underset{H \rightarrow 1}{\longrightarrow}(m-1) ! c_{1} \sigma_{0}^{2(m-1)} t^{m},
$$

which is the $2 \leqslant m$-th cumulant of the $\Gamma\left(c_{1} / \sigma_{0}^{2}, \sigma_{0}^{2} t\right)$ distribution. Since the $\Gamma$ distribution is uniquely determined by its cumulants, we can state the following:

Remark 14. If $H \rightarrow 1$ such that (3.45) holds, then the one-dimensional distribution of the shifted LISDLG process converges to the $\Gamma$ distribution, namely, we have

$$
J(t)+c_{1} t \underset{H \rightarrow 1}{\stackrel{\mathrm{w}}{\longrightarrow}} \Gamma\left(c_{1} / \sigma_{0}^{2}, t \sigma_{0}^{2}\right) .
$$

Equation (3.46) means that the one-dimensional distribution of the $\triangle$ LISDLG process is easily mixed up with a proper $\Gamma$ distribution, leastwise for values of $H$ near 1. See Section 4.2 for a potential occurrence in practice.

\subsection{Spectra of the SDLG process and its offsprings}

The conventional definition of the spectrum of order $m$, see Definition 8 , requires the integrability of the $m$-th order joint cumulant as a function of $m-1$ variables. This condition comes true in the case of the stationary DLG process, e.g. see Lemma 2. However, LRD processes fail to satisfy it by definition, at least for $m=2$. If LRD is expressed also by the higher order cumulants, then the condition for the existence of 
higher order spectra may be unfulfilled. This happens for the SDLG process $Y(t)$, as the following proposition states:

Proposition 18. For each $m \in\{2,3,4, \ldots\}$ the $m$-th order joint cumulant of the SDLG process $Y(t)$, as a function of $m-1$ variables, is not integrable, i.e.

$$
\int_{\mathbb{R}^{m-1}}\left|\operatorname{cum}\left(Y(0), Y\left(t_{1}\right), \ldots, Y\left(t_{m-1}\right)\right)\right| \mathrm{d} \underline{t}_{(m-1)}=\infty
$$

Proof. By formula (3.17),

$$
\begin{aligned}
\int_{\mathbb{R}^{m-1}} & \left|\operatorname{cum}\left(Y(0), Y\left(t_{1}\right), \ldots, Y\left(t_{m-1}\right)\right)\right| \mathrm{d} \underline{t}_{(m-1)} \\
& =K \int_{\mathbb{R}^{m-1}}\left(2 \lambda+\left|t_{1}\right|+\left|t_{2}-t_{1}\right|+\cdots+\left|t_{m-1}-t_{m-2}\right|+\left|t_{m-1}\right|\right)^{2(H-1)} \mathrm{d} \underline{t}_{(m-1)} \\
& \geqslant 2^{2(H-1)} K \int_{\mathbb{R}^{m-1}}\left(\lambda+\left|t_{1}\right|+\left|t_{2}\right|+\cdots+\left|t_{m-1}\right|\right)^{2(H-1)} \mathrm{d} \underline{t}_{(m-1)} \\
& =\infty
\end{aligned}
$$

where $K>0$ is a constant. The inequality in (3.47) is the consequence of the triangle inequality and that $2(H-1)<0$, see Condition 1 . The result of the last integral is infinity because of the Fubini theorem and that $H>1 / 2$, see Condition 1 , too.

Fortunately, the integrability of a function guarantees the one-to-one correspondence between it and its (inverse) Fourier transform. Thus Definition 8 can be generalized adequately for our case, i.e. when the cumulant is not integrable.

Definition 9. Let $m \in\{2,3,4, \ldots\}$. Let $X(t), t \in \mathbb{R}$, be a stationary process with finite absolute moments of order $m$, and let the cumulant function cum $\left(X(0), X\left(t_{1}\right), \ldots, X\left(t_{m-1}\right)\right)$ be given. Assume that the function $S_{m, X}\left(\underline{\omega}_{(m-1)}\right)$ is integrable, and its inverse Fourier transform is just the cumulant function, i.e.

$$
\left.\operatorname{cum}\left(X(0), X\left(t_{1}\right), \ldots, X\left(t_{m-1}\right)\right)=\int_{\mathbb{R}^{m-1}} \exp \left(i \sum_{j=1}^{m-1} t_{j} \omega_{j}\right) S_{m, X} \underline{\underline{\omega}}_{(m-1)}\right) \mathrm{d} \underline{\omega}_{(m-1)}
$$

Then function $S_{m, X}\left(\underline{\omega}_{(m-1)}\right)$ is called the $m$-th order spectrum of process $X(t)$. For $m=2$ and $m=3$ the $m$-th order spectrum is called a spectrum and a bispectrum, respectively.

Thanks to Definition 9 we are now able to give the spectra in a clear form, leastwise the spectrum and the bispectrum.

Proposition 19. The spectrum of the SDLG process $Y(t)$ exists and it can be given either by the mixture representation

$$
S_{2, Y}(\omega)=\mathrm{E}_{A} S_{2, R_{1,1}}(\omega)
$$

or by the following explicit expression.

$$
S_{2, Y}(\omega)=\frac{1}{2 \pi} \cdot \frac{1}{1-H} \mu_{0} \lambda^{3-2 H} \sigma_{0}^{2} \operatorname{Re}\left(\mathrm{e}^{i \lambda \omega} \int_{\lambda}^{\infty} x^{2(H-1)} \mathrm{e}^{-i x \omega} \mathrm{d} x\right) .
$$


Proof. Because of the mixture representation of the cumulant function, see Lemma 4, we have

$$
\begin{aligned}
\operatorname{cum}(Y(0), Y(t)) & =\mathrm{E}_{A} \operatorname{cum}\left(R_{1,1}(0), R_{1,1}(t)\right) \\
& =\mathrm{E}_{A} \int_{\mathbb{R}} \mathrm{e}^{i t \omega} S_{2, R_{1,1}}(\omega) \mathrm{d} \omega \\
& =\int_{\mathbb{R}} \mathrm{e}^{i t \omega} \mathrm{E}_{A} S_{2, R_{1,1}}(\omega) \mathrm{d} \omega \\
& =\frac{1}{2 \pi} \int_{\mathbb{R}} \mathrm{e}^{i t \omega} \mathrm{E}_{A}\left(\frac{2 \mu_{0} \sigma_{0}^{2}}{\omega^{2}+4 \alpha^{2}}\right) \mathrm{d} \omega \\
& =\frac{1}{2 \pi} \int_{\mathbb{R}} \mathrm{e}^{i t \omega} \frac{2 \mu_{0} \sigma_{0}^{2} \lambda^{3-2 H}}{\Gamma(3-2 H)} \int_{0}^{\infty} \frac{1}{\omega^{2}+x^{2}} x^{2(1-H)} \mathrm{e}^{-\lambda x} \mathrm{~d} x \mathrm{~d} \omega \\
& =\frac{1}{2 \pi} \int_{\mathbb{R}} \mathrm{e}^{i t \omega} \frac{1}{1-H} \mu_{0} \lambda^{3-2 H} \sigma_{0}^{2} \operatorname{Re}\left(\mathrm{e}^{i \lambda \omega} \int_{\lambda}^{\infty} \mathrm{e}^{-i s \omega} s^{2(H-1)} \mathrm{d} s\right) \mathrm{d} \omega .
\end{aligned}
$$

The third equation is true because of the Fubini theorem, the fourth is the consequence of Proposition 8 , formula (2.3), Condition 4 and of the fact that $\mu=\mu_{0}$. The fifth equation is motivated by Condition 3 . Define the function

$$
\begin{aligned}
& S_{2, Y}(\omega) \doteq \frac{1}{2 \pi} \cdot \frac{1}{1-H} \mu_{0} \lambda^{3-2 H} \sigma_{0}^{2} \operatorname{Re}\left(\mathrm{e}^{i \lambda \omega} \int_{\lambda}^{\infty} \mathrm{e}^{-i s \omega} s^{2(H-1)} \mathrm{d} s\right) \\
& =\frac{1}{2 \pi} 2 \mu_{0} \sigma_{0}^{2} \frac{\lambda^{3-2 H}}{\Gamma(3-2 H)} \int_{0}^{\infty} \frac{1}{\omega^{2}+x^{2}} x^{2(1-H)} \mathrm{e}^{-\lambda x} \mathrm{~d} x .
\end{aligned}
$$

By the second expression in (3.51), changing the order of the integrals we have

$$
\begin{aligned}
\int_{\mathbb{R}}\left|S_{2, Y}(\omega)\right| \mathrm{d} \omega & =\frac{1}{2 \pi} 2 \mu_{0} \sigma_{0}^{2} \frac{\lambda^{3-2 H}}{\Gamma(3-2 H)} \int_{0}^{\infty} \int_{\mathbb{R}} \frac{1}{\omega^{2}+x^{2}} \mathrm{~d} \omega x^{2(1-H)} \mathrm{e}^{-\lambda x} \mathrm{~d} x \\
& =\mu_{0} \sigma_{0}^{2} \frac{\lambda^{3-2 H}}{\Gamma(3-2 H)} \int_{0}^{\infty} x^{1-2 H} \mathrm{e}^{-\lambda x} \mathrm{~d} x=\mu_{0} \sigma_{0}^{2} \lambda \frac{1}{2(1-H)}<\infty
\end{aligned}
$$

Therefore $S_{2, Y}(\omega)$ is integrable and its inverse Fourier transform is the second order cumulant function, see formula (3.50). Thus, $S_{2, Y}(\omega)$ is really the spectrum of process $Y(t)$, and this proves formula (3.49). Formula (3.48) is the consequence of the third equation in (3.50) and the one-to-one correspondence between the spectrum and the covariance.

Proposition 20. Recall that the LISDLG process $J(t)$ has stationary increments. Thus if one defines the spectrum of the informal derivative process $J^{\prime}=\frac{\mathrm{d}}{\mathrm{d} t} J$ as the limit

$$
S_{2, J^{\prime}}(\omega) \stackrel{\circ}{=} \lim _{\lambda \rightarrow 0} S_{2, Y_{\lambda}}(\omega)
$$

then one has

$$
S_{2, J^{\prime}}(\omega)=\frac{1}{2 \cos (H \pi) \Gamma(3-2 H)} c_{0} \sigma_{0}^{2}|\omega|^{1-2 H}=\frac{1}{2 \pi} \cdot \frac{\Gamma(2 H-1)}{1-H} c_{0} \sigma_{0}^{2} \sin (H \pi)|\omega|^{1-2 H} .
$$

We will call $S_{2, J^{\prime}}(\omega)$ the generalized spectrum (appertaining to process $J(t)$ [to avoid reference to the informal derivative process $\left.J^{\prime}\right]$ ). 
Proof. Applying Proposition 19 and Condition 7 we have

$$
\begin{aligned}
S_{2, J^{\prime}}(\omega) & =\lim _{\lambda \rightarrow 0} S_{2, Y_{\lambda}}(\omega)=\frac{1}{2 \pi} \cdot \frac{1}{1-H} c_{0} \sigma_{0}^{2} \operatorname{Re}\left(\int_{0}^{\infty} \mathrm{e}^{-i s \omega} s^{2(H-1)} \mathrm{d} s\right) \\
& =\frac{1}{2 \pi} \cdot \frac{1}{1-H} c_{0} \sigma_{0}^{2} \operatorname{Re}\left(\Gamma(2 H-1)(i \omega)^{1-2 H}\right)=\frac{1}{2 \pi} \cdot \frac{\Gamma(2 H-1)}{1-H} c_{0} \sigma_{0}^{2} \sin (H \pi)|\omega|^{1-2 H},
\end{aligned}
$$

where the third equation is the consequence of formula (2.3.3.1) in [30]. By the identity

$$
\Gamma(2 H-1) \Gamma(2(1-H))=-\frac{\pi}{\sin (2 \pi H)},
$$

one obtains the constant multiplier in the required form.

Remark 15. In view of Corollary 5 , it is not surprising that, apart from a constant multiplier, $S_{2, J^{\prime}}(\omega)$ in Corollary 20 is the same as the informal spectrum of the continuous time FGN, i.e. the informal spectrum of the informal derivative of the FBM.

Let us consider the analogous statements for the bispectrum.

Proposition 21. The bispectrum of the SDLG process $Y(t)$ exists and it can be given either by the mixture representation

$$
S_{3, Y}\left(\underline{\omega}_{(2)}\right)=\mathrm{E}_{A} S_{3, R_{1,1}}\left(\underline{\omega}_{(2)}\right)
$$

or in the following explicit manner.

$$
S_{3, Y}\left(\underline{\omega}_{(2)}\right)=-\left(\frac{1}{2 \pi}\right)^{2} \frac{6 \mu_{0} \lambda^{3-2 H}}{1-H} \sigma_{0}^{4} \frac{1}{\omega_{1} \omega_{2} \omega_{3}} \operatorname{Im} \underset{\left.\underline{(}_{(3)}\right)}{\operatorname{sym}}\left(\omega_{1}^{2} \int_{\lambda}^{\infty} \mathrm{e}^{i(\lambda-s) \omega_{1}} s^{2(H-1)} \mathrm{d} s\right),
$$

where $\omega_{3} \stackrel{\circ}{=}-\omega_{1}-\omega_{2}$.

The bispectrum $S_{3, Y}\left(\underline{\omega}_{(2)}\right)$ has the extraordinary property that it is real-valued moreover it is positive. Proof. Because of the mixture representation of the cumulant function, see Lemma 4, we have

$$
\begin{aligned}
\operatorname{cum}\left(Y(0), Y\left(t_{1}\right), Y\left(t_{2}\right)\right) & =\mathrm{E}_{A} \operatorname{cum}\left(R_{1,1}(0), R_{1,1}\left(t_{1}\right), R_{1,1}\left(t_{2}\right)\right) \\
& =\mathrm{E}_{A} \int_{\mathbb{R}^{2}} \mathrm{e}^{i\left(t_{1} \omega_{1}+t_{2} \omega_{2}\right)} S_{3, R_{1,1}}\left(\omega_{1}, \omega_{2}\right) \mathrm{d} \underline{\omega}_{(2)}=\int_{\mathbb{R}^{2}} \mathrm{e}^{i\left(t_{1} \omega_{1}+t_{2} \omega_{2}\right)} \mathrm{E}_{A} S_{3, R_{1,1}}\left(\underline{\omega}_{(2)}\right) \mathrm{d} \underline{\omega}_{(2)} \\
& =\left(\frac{1}{2 \pi}\right)^{2} \int_{\mathbb{R}^{2}} \mathrm{e}^{i\left(t_{1} \omega_{1}+t_{2} \omega_{2}\right)} 12 \mu_{0} \sigma_{0}^{4} \mathrm{E}_{A}\left(\frac{1}{-2 \alpha} \underset{\left(\underline{\omega}_{(3)}\right)}{\operatorname{sym}}\left(\frac{1}{-i \omega_{1}-2 \alpha} \cdot \frac{1}{i \omega_{2}-2 \alpha}\right)\right) \mathrm{d} \underline{\omega}_{(2)} .
\end{aligned}
$$

The third equation is due to the Fubini theorem, and the fourth is the consequence of Proposition 9, formula (2.28), Condition 4 , and of the fact that $\mu=\mu_{0}$. Let us define the function

$$
\begin{aligned}
S_{3, Y}\left(\underline{\omega}_{(2)}\right) & \stackrel{\circ}{=} \mathrm{E}_{A} S_{3, R_{1,1}}\left(\underline{\omega}_{(2)}\right) \\
& =\left(\frac{1}{2 \pi}\right)^{2} 12 \mu_{0} \sigma_{0}^{4} \underset{\left(\underline{\omega}_{(3)}\right)}{\operatorname{sym}} \mathrm{E}_{A}\left(\frac{1}{-2 \alpha} \cdot \frac{1}{-i \omega_{1}-2 \alpha} \cdot \frac{1}{i \omega_{2}-2 \alpha}\right)
\end{aligned}
$$


Notice that $S_{3, Y}\left(\underline{\omega}_{(2)}\right)$ must be real-valued and positive, because for each realization of the random variable $-2 \alpha$, so is the bispectrum $S_{3, R_{1,1}}\left(\underline{\omega}_{(2)}\right)$. Now we have to calculate the expectation

$$
\mathrm{E}\left(\frac{1}{-2 \alpha} \cdot \frac{1}{-i \omega_{1}-2 \alpha} \cdot \frac{1}{i \omega_{2}-2 \alpha}\right)
$$

where $-2 \alpha \sim \Gamma(3-2 H, 1 / \lambda)$, see Condition 3. Function $1 /(i \omega-2 \alpha), \omega \in \mathbb{R}$, is just the Fourier transform of function $\exp (2 \alpha t), t \geqslant 0$, and the latter is more usable from the point of view of the $\Gamma$ distribution. Using this observation, and partial fraction decomposition, one can easily obtain

$$
\mathrm{E}\left(\frac{1}{-2 \alpha} \cdot \frac{1}{-i \omega_{1}-2 \alpha} \cdot \frac{1}{i \omega_{2}-2 \alpha}\right)=\lambda^{3-2 H} \frac{1}{\omega_{1} \omega_{2}} \int_{\lambda}^{\infty}\left(1-\frac{\omega_{2}}{\omega_{1}+\omega_{2}} \mathrm{e}^{-i(\lambda-s) \omega_{1}}-\frac{\omega_{1}}{\omega_{1}+\omega_{2}} \mathrm{e}^{i(\lambda-s) \omega_{2}}\right) s^{2 H-3} \mathrm{~d} s .
$$

Substituting this expression into (3.57), we have

$$
\begin{aligned}
& S_{3, Y}\left(\underline{\omega}_{(2)}\right) \\
& =\left(\frac{1}{2 \pi}\right)^{2} 12 \mu_{0} \lambda^{3-2 H} \sigma_{0}^{4} \underset{\left(\underline{\omega}_{(3)}\right)}{\operatorname{sym}}\left(\frac{1}{\omega_{1} \omega_{2}} \int_{\lambda}^{\infty}\left(1-\frac{\omega_{2}}{\omega_{1}+\omega_{2}} \mathrm{e}^{-i(\lambda-s) \omega_{1}}-\frac{\omega_{1}}{\omega_{1}+\omega_{2}} \mathrm{e}^{i(\lambda-s) \omega_{2}}\right) s^{2 H-3} \mathrm{~d} s\right) \\
& =\left(\frac{1}{2 \pi}\right)^{2} 12 \mu_{0} \lambda^{3-2 H} \sigma_{0}^{4} \frac{1}{\omega_{1} \omega_{2} \omega_{3}} \int_{\lambda}^{\infty} \operatorname{sym}_{\left(\underline{\omega}_{(3)}\right)}^{\infty}\left(-\frac{\omega_{2} \omega_{3}}{\omega_{1}+\omega_{2}} \mathrm{e}^{-i(\lambda-s) \omega_{1}}-\frac{\omega_{1} \omega_{3}}{\omega_{1}+\omega_{2}} \mathrm{e}^{i(\lambda-s) \omega_{2}}\right) s^{2 H-3} \mathrm{~d} s \\
& =\left(\frac{1}{2 \pi}\right)^{2} 12 \mu_{0} \lambda^{3-2 H} \sigma_{0}^{4} \frac{1}{\omega_{1} \omega_{2} \omega_{3}} \int_{\lambda}^{\infty} \operatorname{sym}_{\left(\underline{\omega}_{(3)}\right)}^{\infty}\left(\omega_{2} \mathrm{e}^{i(\lambda-s) \omega_{1}}+\omega_{1} \mathrm{e}^{i(\lambda-s) \omega_{2}}\right) s^{2 H-3} \mathrm{~d} s \\
& =\left(\frac{1}{2 \pi}\right)^{2} 12 \mu_{0} \lambda^{3-2 H} \sigma_{0}^{4} \frac{1}{\omega_{1} \omega_{2} \omega_{3}} \int_{\lambda}^{\infty} \operatorname{sym}_{\left(\underline{\omega}_{(3)}\right)}^{\infty}\left(-\omega_{1} \mathrm{e}^{i(\lambda-s) \omega_{1}}\right) s^{2 H-3} \mathrm{~d} s .
\end{aligned}
$$

Integrating by parts changes (3.58) into

$$
S_{3, Y}\left(\underline{\omega}_{(2)}\right)=-\left(\frac{1}{2 \pi}\right)^{2} \frac{6 \mu_{0} \lambda^{3-2 H}}{1-H} \sigma_{0}^{4} \frac{1}{\omega_{1} \omega_{2} \omega_{3}} \underset{\left(\underline{\omega}_{(3)}\right)}{\operatorname{Im}} \operatorname{sym}_{\lambda}\left(\omega_{1}^{2} \int_{\lambda}^{\infty} \mathrm{e}^{i(\lambda-s) \omega_{1}} s^{2(H-1)} \mathrm{d} s\right) .
$$

What is left to prove is that $S_{3, Y}\left(\underline{\omega}_{(2)}\right)$ is integrable.

$$
\begin{aligned}
\int_{\mathbb{R}^{2}}\left|S_{3, Y}\left(\underline{\omega}_{(2)}\right)\right| \mathrm{d} \underline{\omega}_{(2)} & =\int_{\mathbb{R}^{2}} S_{3, Y}\left(\underline{\omega}_{(2)}\right) \mathrm{d} \underline{\omega}_{(2)}=\int_{\mathbb{R}^{2}} \mathrm{E}_{A} S_{3, R_{1,1}}\left(\underline{\omega}_{(2)}\right) \mathrm{d} \underline{\omega}_{(2)} \\
& =\mathrm{E}_{A} \int_{\mathbb{R}^{2}} S_{3, R_{1,1}}\left(\underline{\omega}_{(2)}\right) \mathrm{d} \underline{\omega}_{(2)}=\mathrm{E}_{A} \operatorname{cum}_{3}\left(R_{1,1}(0)\right)=2 \mu_{0} \sigma_{0}^{4} \mathrm{E}_{A} \frac{1}{-2 \alpha} \\
& =\mu_{0} \sigma_{0}^{4} \frac{\lambda}{1-H}<\infty .
\end{aligned}
$$

Here the first equation is the consequence of the positivity of $S_{3, Y}\left(\underline{\omega}_{(2)}\right)$, the second is true because of (3.57), the third is the Fubini theorem, the fourth is the consequence of Definition 8 , the fifth is true because of Proposition 6, Condition 4 and of the fact that $\mu=\mu_{0}$. The sixth equation is motivated by Condition 3 , and the finiteness arises from inequality $H<1$, see Condition 1 . 
Proposition 22. If one defines the bispectrum of the informal derivative process $J^{\prime}=\frac{\mathrm{d}}{\mathrm{d} t} J$ as the limit

$$
S_{3, J^{\prime}}\left(\underline{\omega}_{(2)}\right) \doteq \lim _{\lambda \rightarrow 0} S_{3, Y_{\lambda}}\left(\underline{\omega}_{(2)}\right)
$$

then one obtains

$$
S_{3, J^{\prime}}\left(\underline{\omega}_{(2)}\right)=\left(\frac{1}{2 \pi}\right)^{2} \frac{6 \Gamma(2 H-1)}{1-H} c_{0} \sigma_{0}^{4} \frac{1}{\omega_{1} \omega_{2} \omega_{3}} \underset{\left.\underline{(}_{(3)}\right)}{\operatorname{Im}} \operatorname{sym}_{\left(\left(i \omega_{1}\right)^{3-2 H}\right),}
$$

or, in another form,

$$
S_{3, J^{\prime}}\left(\underline{\omega}_{(2)}\right)=\frac{1}{2 \pi} \cdot \frac{3}{\sin (H \pi) \Gamma(3-2 H)} c_{0} \sigma_{0}^{4} \underset{\left(\underline{\omega}_{(3)}\right)}{\operatorname{sym}} \frac{\left|\omega_{1}\right|^{2(1-H)}}{\omega_{2} \omega_{3}}
$$

where $\omega_{3} \stackrel{\circ}{=}-\omega_{1}-\omega_{2}$.

We will call $\left.S_{3, J^{\prime}} \underline{\underline{\omega}}_{(2)}\right)$ the generalized bispectrum (appertaining to process $J(t)$ [to avoid reference to the informal derivative process $\left.J^{\prime}\right]$ ).

The generalized bispectrum $S_{3, J^{\prime}}\left(\underline{\omega}_{(2)}\right)$ has the extraordinary property that it is real-valued moreover it is positive.

Proof. The bispectrum of the SDLG process $Y(t)$ is

$$
S_{3, Y}\left(\underline{\omega}_{(2)}\right)=-\left(\frac{1}{2 \pi}\right)^{2} \frac{6 \mu_{0} \lambda^{3-2 H}}{1-H} \sigma_{0}^{4} \frac{1}{\omega_{1} \omega_{2} \omega_{3}} \operatorname{Im} \underset{\left(\underline{\omega}_{(3)}\right)}{\operatorname{sym}}\left(\omega_{1}^{2} \int_{\lambda}^{\infty} \mathrm{e}^{i(\lambda-s) \omega_{1}} s^{2(H-1)} \mathrm{d} s\right)
$$

see (3.56). Though in (3.62) the function to be integrated is not Lebesgue integrable on the whole positive ray, the integral exists in the sense of principal value. In particular, the following integral is defined, and there is an explicit expression for it, i.e.

$$
\int_{0}^{\infty} \mathrm{e}^{-i s \omega} s^{2(H-1)} \mathrm{d} s \stackrel{\circ}{=} \lim _{N \rightarrow \infty} \int_{0}^{N} \mathrm{e}^{-i s \omega} s^{2(H-1)} \mathrm{d} s=\Gamma(2 H-1)(i \omega)^{1-2 H},
$$

$\omega \neq 0$, see formula (2.3.3.1) in [30]. By (3.59) we have to take the limit as $\lambda \rightarrow 0$ in (3.62). To do this, cut the integral of (3.62) into two parts:

$$
\int_{\lambda}^{\infty} \mathrm{e}^{i(\lambda-s) \omega} s^{2(H-1)} \mathrm{d} s=\int_{\lambda}^{1} \mathrm{e}^{i(\lambda-s) \omega} s^{2(H-1)} \mathrm{d} s+\mathrm{e}^{i \lambda \omega} \int_{1}^{\infty} \mathrm{e}^{-i s \omega} s^{2(H-1)} \mathrm{d} s .
$$

By Condition 7 and (3.64) the convergence of (3.62) to (3.59) is now obvious. In another form we have

$$
S_{3, J^{\prime}}\left(\underline{\omega}_{(2)}\right)=\frac{1}{2 \pi} \cdot \frac{3}{\sin (H \pi) \Gamma(3-2 H)} c_{0} \sigma_{0}^{4} \underset{\left(\underline{\omega}_{(3)}\right)}{\operatorname{sym}} \frac{\left|\omega_{1}\right|^{2(1-H)}}{\omega_{2} \omega_{3}},
$$

where we also applied identity (3.54).

The fact that $S_{3, J^{\prime}}\left(\underline{\omega}_{(2)}\right)$ is real-valued and positive, follows from its form (3.61) since

$$
\underset{\left(\underline{\omega}_{(3)}\right)}{\operatorname{sym}} \frac{\left|\omega_{1}\right|^{2(1-H)}}{\omega_{2} \omega_{3}}=\frac{\left|\omega_{1}\right|^{3-2 H} \operatorname{sig}\left(\omega_{1}\right)+\left|\omega_{2}\right|^{3-2 H} \operatorname{sig}\left(\omega_{2}\right)+\left|\omega_{3}\right|^{3-2 H} \operatorname{sig}\left(\omega_{3}\right)}{3 \omega_{1} \omega_{2} \omega_{3}}>0
$$


for all $\omega_{1}, \omega_{2} \in \mathbb{R}$ for which $\omega_{1} \omega_{2} \omega_{3} \neq 0$. The inequality in (3.65) is easily obtainable supposing without loss of generality that $\omega_{1} \omega_{2}>0$ and using the inequality $(x+1)^{3-2 H}>1+x^{3-2 H}$ with $x=\left|\omega_{2} / \omega_{1}\right|$.

Remark 16. The generalized bispectrum $S_{3, J^{\prime}}\left(\underline{\omega}_{(2)}\right)$ behaves around zero as

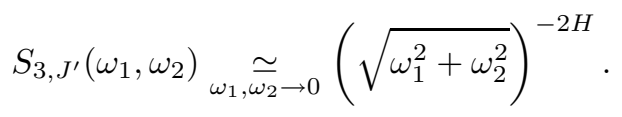

Remark 17. The property of the bispectrum $S_{3, Y}\left(\underline{\omega}_{(2)}\right)$ that it is real-valued, is equivalent to the property of the cumulants that

$$
\operatorname{cum}\left(Y(0), Y\left(t_{1}\right), Y\left(t_{2}\right)\right)=\operatorname{cum}\left(Y(0), Y\left(t_{2}-t_{1}\right), Y\left(t_{2}\right)\right)
$$

$t_{1}, t_{2} \in \mathbb{R}$

Proof. By the stationarity we have

$$
\operatorname{cum}\left(Y(0), Y\left(-t_{1}\right), Y\left(-t_{2}\right)\right)=\operatorname{cum}\left(Y\left(t_{2}\right), Y\left(t_{2}-t_{1}\right), Y(0)\right)=\operatorname{cum}\left(Y(0), Y\left(t_{2}-t_{1}\right), Y\left(t_{2}\right)\right) .
$$

If the bispectrum is real, then by Definition 9 we have

$$
\operatorname{cum}\left(Y(0), Y\left(t_{1}\right), Y\left(t_{2}\right)\right)=\operatorname{cum}\left(Y(0), Y\left(-t_{1}\right), Y\left(-t_{2}\right)\right) .
$$

Comparing (3.68) and (3.67) we have (3.66).

On the other hand, if (3.66) holds, then by Definition 9 we have

$$
\int_{\mathbb{R}^{2}} \mathrm{e}^{i\left(t_{1} \omega_{1}+t_{2} \omega_{2}\right)} S_{3, Y}\left(\underline{\omega}_{(2)}\right) \mathrm{d} \underline{\omega}_{(2)}=\int_{\mathbb{R}^{2}} \mathrm{e}^{i\left(t_{1} \omega_{1}+t_{2} \omega_{2}\right)} \overline{S_{3, Y}\left(\underline{\omega}_{(2)}\right)} \mathrm{d} \underline{\omega}_{(2)},
$$

for all $t_{1}, t_{2} \in \mathbb{R}$. There is a one-to-one correspondence between integrable functions and their inverse Fourier transforms, thus by (3.69) the bispectrum must be real.

Incidentally, equation (3.66) is also a consequence of the form of the cumulants, see Lemma 4 . More generally, the following statement holds true.

Remark 18. We have

$$
\operatorname{cum}\left(Y(0), Y\left(t_{1}\right), \ldots, Y\left(t_{m-1}\right)\right)=\operatorname{cum}\left(Y(0), Y\left(t_{m-1}-t_{m-2}\right), \ldots, Y\left(t_{m-1}\right)\right)
$$

for all $m \geq 3$ and $t_{1}, \ldots, t_{m-1} \in \mathbb{R}$.

Proof. The terms in the sum in (3.17) correspond to circles $\left(Y(0), Y\left(t_{i_{1}}\right), \ldots, Y\left(t_{i_{m-1}}\right), Y(0)\right)$, and any such term remains the same if one walks round the circle in the opposite direction.

In practice it is usually not the whole process but its discretely sampled version that is observed. For the LISDLG process $J(t)$ this means that we deal with the discrete time LISDLG process $J(t), t \in \mathbb{Z}$, say, or equivalently, with the $\Delta$ LISDLG process $\Delta J(t)=J(t+1)-J(t), t \in \mathbb{Z}$. The latter is more desirable for the very reason that it is stationary. Unfortunately, its continuous time counterpart, the derivative process $J^{\prime}(t)$ does not exist, and thus there is another reason why we have to deal with the $\Delta$ LISDLG process $\Delta J(t)$, $t \in \mathbb{Z}$. On the other hand, we have seen above that the bispectrum of the SDLG process $Y_{\lambda}(t)$ is real-valued and positive. We will state that the discrete time counterpart of the SDLG process $Y_{\lambda}(t), t \in \mathbb{R}$, i.e. the $\Delta$ ISDLG process $\Delta J_{\lambda}(t), t \in \mathbb{Z}$, has a bispectrum that is also real-valued and positive. Going further and taking the limit as $\lambda \rightarrow 0$, we arrive at the $\Delta$ LISDLG process $\Delta J(t), t \in \mathbb{Z}$, and we find that it also has a bispectrum which is real-valued and positive too.

It is important to remark that in the case of discrete time LRD processes we will use the notion of spectrum of any order in the same sense as in the case of continuous time LRD processes. That is, even if the cumulant series is not absolutely summable, the spectrum must be integrable and its inverse Fourier transform 
must be the cumulant series. Clearly, the integrability of the spectrum ensures - also in the discrete-time case - a one-to-one correspondence between the spectrum and the cumulant series.

Let us examine first the second order spectrum.

Proposition 23. The spectrum of the $\Delta$ ISDLG process $\Delta J_{\lambda}(t)$ exists and it is

$$
\begin{aligned}
S_{2, \Delta J_{\lambda}}(\omega) & =\left|\mathrm{e}^{i \omega}-1\right|^{2} \sum_{k=-\infty}^{\infty}\left(\left(\omega^{(k)}\right)^{-2} S_{2, Y_{\lambda}}\left(\omega^{(k)}\right)\right) \\
& =\frac{1}{2 \pi} \cdot \frac{\mu_{0} \lambda^{3-2 H}}{1-H} \sigma_{0}^{2}\left|\mathrm{e}^{i \omega}-1\right|^{2} \operatorname{Re}\left(\sum_{k=-\infty}^{\infty}\left(\left(\omega^{(k)}\right)^{-2} \mathrm{e}^{i \lambda \omega^{(k)}} \int_{\lambda}^{\infty} \mathrm{e}^{-i x \omega^{(k)}} x^{2(H-1)} \mathrm{d} x\right)\right),
\end{aligned}
$$

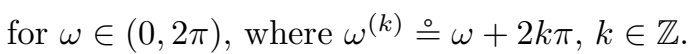

Proof. Let us calculate cum $\left(\Delta J_{\lambda}(0), \Delta J_{\lambda}(0)\right)$. At first, we can change the order of the cumulant operator and the integrals by right of a similar reasoning as in (2.26) (see the explanation after (2.26)). Then applying Lemma 4 we have

$$
\begin{aligned}
\operatorname{cum}\left(\Delta J_{\lambda}(0), \Delta J_{\lambda}(0)\right) & =\operatorname{cum}\left(\int_{0}^{1} Y_{\lambda}(s) \mathrm{d} s, \int_{0}^{1} Y_{\lambda}(s) \mathrm{d} s\right) \\
& =\int_{0}^{1} \int_{0}^{1} \operatorname{cum}\left(Y_{\lambda}\left(s_{0}\right), Y_{\lambda}\left(s_{1}\right)\right) \mathrm{d} s_{1} \mathrm{~d} s_{0} \\
& =\int_{0}^{1} \int_{0}^{1} \sigma_{0}^{2} \frac{\mu_{0} \lambda}{2(1-H)}\left(1+\frac{D_{\tau}(\underline{s})}{2 \lambda}\right)^{2(H-1)} \mathrm{d} s_{1} \mathrm{~d} s_{0} \\
& =\frac{1}{2(1-H)} \sigma_{0}^{2} \mu_{0} \lambda^{3-2 H} \int_{0}^{1} \int_{0}^{1}\left(\lambda+\left|s_{1}-s_{0}\right|\right)^{2(H-1)} \mathrm{d} s_{1} \mathrm{~d} s_{0} \\
& =\frac{1}{(1-H)(2 H-1)} \sigma_{0}^{2} \mu_{0} \lambda^{3-2 H}\left(\frac{1}{2 H}\left((\lambda+1)^{2 H}-\lambda^{2 H}\right)-\lambda^{2 H-1}\right) .
\end{aligned}
$$

We will need the resulting expression later, but presently we only use the fact that $\operatorname{cum}\left(\Delta J_{\lambda}(0), \Delta J_{\lambda}(0)\right)$ is finite.

Now let us calculate cum $\left(\Delta J_{\lambda}(0), \Delta J_{\lambda}(t)\right)$ in general. We can change the order of the cumulant operator and the integrals as above, so by the stationarity we have

$$
\begin{aligned}
\operatorname{cum}\left(\Delta J_{\lambda}(0), \Delta J_{\lambda}(t)\right) & =\operatorname{cum}\left(\int_{0}^{1} Y_{\lambda}(s) \mathrm{d} s, \int_{t}^{t+1} Y_{\lambda}(s) \mathrm{d} s\right) \\
& =\int_{\substack{0 \\
\left(s_{0}\right)\left(s_{1}\right)}}^{1} \int_{t}^{t+1} \operatorname{cum}\left(Y_{\lambda}(0), Y_{\lambda}\left(s_{1}-s_{0}\right)\right) \mathrm{d} s_{1} \mathrm{~d} s_{0} .
\end{aligned}
$$


We know that $S_{2, Y_{\lambda}}(\omega)$ is the spectrum of the continuous time process $Y_{\lambda}(t)$, therefore continuing (3.71), we obtain

$$
\begin{aligned}
\operatorname{cum}\left(\Delta J_{\lambda}(0), \Delta J_{\lambda}(t)\right) & =\int_{\substack{0 \\
\left(s_{0}\right)\left(s_{1}\right)(\omega)}}^{1} \int_{\mathbb{R}}^{t+1} \mathrm{e}^{i\left(s_{1}-s_{0}\right) \omega} S_{2, Y_{\lambda}}(\omega) \mathrm{d} \omega \mathrm{d} s_{1} \mathrm{~d} s_{0} \\
& =\int_{\mathbb{R}}^{1} \int_{0}^{t+1} \int_{t}^{t+1\left(s_{1}-s_{0}\right) \omega} \mathrm{d} s_{1} \mathrm{~d} s_{0} S_{2, Y_{\lambda}}(\omega) \mathrm{d} \omega,
\end{aligned}
$$

where the change of the order of the integrals is the consequence of the integrability of $S_{2, Y_{\lambda}}(\omega)$. Calculating the two inner integrals in the last row of (3.72), and breaking the outer integral and performing the proper substitutions yields

$$
\begin{aligned}
\operatorname{cum}\left(\Delta J_{\lambda}(0), \Delta J_{\lambda}(t)\right) & =\int_{\mathbb{R}} \mathrm{e}^{i t \omega} \omega^{-2}\left|\mathrm{e}^{i \omega}-1\right|^{2} S_{2, Y_{\lambda}}(\omega) \mathrm{d} \omega \\
& =\sum_{k=-\infty}^{\infty} \int_{0}^{2 \pi} \mathrm{e}^{i t \omega}\left(\omega^{(k)}\right)^{-2}\left|\mathrm{e}^{i \omega}-1\right|^{2} S_{2, Y_{\lambda}}\left(\omega^{(k)}\right) \mathrm{d} \omega .
\end{aligned}
$$

For $t=0$ the above double integral (i.e. a sum and an integral) is finite, because cum $\left(\Delta J_{\lambda}(0), \Delta J_{\lambda}(0)\right)$ is finite. Thus by the Fubuni theorem we can change the order of the sum and the integral in (3.73) to get

$$
\operatorname{cum}\left(\Delta J_{\lambda}(0), \Delta J_{\lambda}(t)\right)=\int_{0}^{2 \pi} \mathrm{e}^{i t \omega}\left|\mathrm{e}^{i \omega}-1\right|^{2} \sum_{k=-\infty}^{\infty}\left(\left(\omega^{(k)}\right)^{-2} S_{2, Y_{\lambda}}\left(\omega^{(k)}\right)\right) \mathrm{d} \omega
$$

Let us define the function

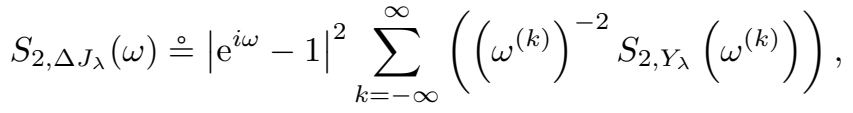

$\omega \in(0,2 \pi)$. We have just mentioned the integrability of $S_{2, \Delta J_{\lambda}}(\omega)$, thus the spectrum of the discrete time process $\Delta J_{\lambda}(t)$ really exists and by $(3.74)$ it is the above-defined $S_{2, \Delta J_{\lambda}}(\omega)$. Moreover, by substituting the form (3.49) of $S_{2, Y_{\lambda}}(\omega)$ into (3.75), we have

$$
S_{2, \Delta J_{\lambda}}(\omega)=\frac{1}{2 \pi} \cdot \frac{\mu_{0} \lambda^{3-2 H}}{1-H} \sigma_{0}^{2}\left|\mathrm{e}^{i \omega}-1\right|^{2} \sum_{k=-\infty}^{\infty}\left(\left(\omega^{(k)}\right)^{-2} \operatorname{Re}\left(\mathrm{e}^{i \lambda \omega^{(k)}} \int_{\lambda}^{\infty} \mathrm{e}^{-i x \omega^{(k)}} x^{2(H-1)} \mathrm{d} x\right)\right) .
$$

The following technical concept will be needed in some of subsequent proofs:

Definition 10. We will say that a set of functions $f_{\lambda}(\omega)$ converges as $\lambda \rightarrow 0$ essentially monotonely to a function $f(\omega)$, if there exist a set of constants $c_{\lambda}$, a constant $c$, a set of functions $g_{\lambda}(\omega)$ and a function $g(\omega)$ such that $f_{\lambda}(\omega)=c_{\lambda} g_{\lambda}(\omega)$ for all $\lambda$ and $\omega, f(\omega)=c g(\omega)$ for all $\omega$, moreover $c_{\lambda} \underset{\lambda \rightarrow 0}{\longrightarrow} c$, and the convergence $f_{\lambda}(\omega) \underset{\lambda \rightarrow 0}{\longrightarrow} f(\omega)$ is monotone (either increasing for all $\omega$ or decreasing for all $\omega$ ). 
Theorem 9. The spectrum of the $\Delta L I S D L G$ process $\Delta J(t)$ exists and it is

$$
\begin{aligned}
S_{2, \Delta J}(\omega) & =\left|\mathrm{e}^{i \omega}-1\right|^{2} \sum_{k=-\infty}^{\infty}\left(\left(\omega^{(k)}\right)^{-2} S_{2, J^{\prime}}\left(\omega^{(k)}\right)\right) \\
& =\frac{1}{2 \pi} \cdot \frac{-\Gamma(2 H-1)}{1-H} c_{0} \sigma_{0}^{2}\left|\mathrm{e}^{i \omega}-1\right|^{2} \operatorname{Re}\left(\sum_{k=-\infty}^{\infty}\left(i \omega^{(k)}\right)^{-1-2 H}\right) \\
& =\frac{1}{2 \pi} \cdot \frac{\Gamma(2 H-1)}{1-H} \sin (H \pi) c_{0} \sigma_{0}^{2}\left|\mathrm{e}^{i \omega}-1\right|^{2} \sum_{k=-\infty}^{\infty}\left|\omega^{(k)}\right|^{-1-2 H}
\end{aligned}
$$

for $\omega \in(0,2 \pi)$, where $\omega^{(k)} \stackrel{\circ}{=} \omega+2 k \pi, k \in \mathbb{Z}$.

Proof. By (3.51),

$$
S_{2, Y_{\lambda}}(\omega)=\frac{1}{2 \pi} \cdot \frac{2}{\Gamma(3-2 H)} \mu_{0} \lambda^{3-2 H} \sigma_{0}^{2} \int_{0}^{\infty} x^{2(1-H)} \mathrm{e}^{-\lambda x} \frac{1}{\omega^{2}+x^{2}} \mathrm{~d} x
$$

thus by (3.52) and Condition 7

$$
S_{2, J^{\prime}}(\omega)=\lim _{\lambda \rightarrow 0} S_{2, Y_{\lambda}}(\omega)=\frac{1}{2 \pi} \cdot \frac{2}{\Gamma(3-2 H)} c_{0} \sigma_{0}^{2} \int_{0}^{\infty} x^{2(1-H)} \frac{1}{\omega^{2}+x^{2}} \mathrm{~d} x
$$

Moreover, the convergence

$$
\int_{0}^{\infty} x^{2(1-H)} \mathrm{e}^{-\lambda x} \frac{1}{\omega^{2}+x^{2}} \mathrm{~d} x \underset{\lambda \rightarrow 0}{\longrightarrow} \int_{0}^{\infty} x^{2(1-H)} \frac{1}{\omega^{2}+x^{2}} \mathrm{~d} x
$$

is monotone, that is, for each $\omega \neq 0$, the left-hand side of (3.78) increases as $\lambda$ decreases. So thus, the convergence in (3.77) is essentially monotone.

Now, by Proposition 23 and formula (3.53),

$$
\begin{aligned}
\lim _{\lambda \rightarrow 0} S_{2, \Delta J_{\lambda}}(\omega) & =\left|\mathrm{e}^{i \omega}-1\right|^{2} \lim _{\lambda \rightarrow 0} \sum_{k=-\infty}^{\infty}\left(\left(\omega^{(k)}\right)^{-2} S_{2, Y_{\lambda}}\left(\omega^{(k)}\right)\right) \\
& =\left|\mathrm{e}^{i \omega}-1\right|^{2} \sum_{k=-\infty}^{\infty}\left(\left(\omega^{(k)}\right)^{-2} S_{2, J^{\prime}}\left(\omega^{(k)}\right)\right) \\
& =\frac{1}{2 \pi} \cdot \frac{\Gamma(2 H-1)}{1-H} \sin (H \pi) c_{0} \sigma_{0}^{2}\left|\mathrm{e}^{i \omega}-1\right|^{2} \sum_{k=-\infty}^{\infty}\left|\omega^{(k)}\right|^{-1-2 H}
\end{aligned}
$$

which is finite for all $\omega \in(0,2 \pi)$. In (3.79) the change of order of the limit and the infinite sum can be justified by the Beppo Levi theorem and the fact that the convergence (3.77) is essentially monotone. Therefore let us define the function

$$
S_{2, \Delta J}(\omega) \stackrel{\circ}{=} \lim _{\lambda \rightarrow 0} S_{2, \Delta J_{\lambda}}(\omega),
$$


$\omega \in(0,2 \pi)$. Then $S_{2, \Delta J}(\omega)$ is integrable, because by the Fatou lemma we have

$$
\begin{aligned}
\int_{0}^{2 \pi}\left|S_{2, \Delta J}(\omega)\right| \mathrm{d} \omega & =\int_{0}^{2 \pi} \underline{\lim }_{\lambda \rightarrow 0}\left|S_{2, \Delta J_{\lambda}}(\omega)\right| \mathrm{d} \omega \\
& \leqslant \frac{\lim _{\lambda \rightarrow 0}}{0} \int_{0}^{2 \pi} S_{2, \Delta J_{\lambda}}(\omega) \mathrm{d} \omega \\
& =\varliminf_{\lambda \rightarrow 0} \operatorname{cum}\left(\Delta J_{\lambda}(0), \Delta J_{\lambda}(0)\right) \\
& =\underline{\lim _{\lambda \rightarrow 0}}\left(\frac{1}{(1-H)(2 H-1)} \sigma_{0}^{2} \mu_{0} \lambda^{3-2 H}\left(\frac{1}{2 H}\left((\lambda+1)^{2 H}-\lambda^{2 H}\right)-\lambda^{2 H-1}\right)\right) \\
& =\frac{1}{(1-H)(2 H-1) 2 H} \sigma_{0}^{2} c_{0}<\infty .
\end{aligned}
$$

The fourth row in (3.81) is the consequence of formula (3.70). Moreover,

$$
\begin{aligned}
\int_{0}^{2 \pi} \mathrm{e}^{i t \omega} S_{2, \Delta J}(\omega) \mathrm{d} \omega & =\int_{0}^{2 \pi} \mathrm{e}^{i t \omega} \lim _{\lambda \rightarrow 0} S_{2, \Delta J_{\lambda}}(\omega) \mathrm{d} \omega \\
& =\lim _{\lambda \rightarrow 0} \int_{0}^{2 \pi} \mathrm{e}^{i t \omega} S_{2, \Delta J_{\lambda}}(\omega) \mathrm{d} \omega=\lim _{\lambda \rightarrow 0} \operatorname{cum}\left(\Delta J_{\lambda}(0), \Delta J_{\lambda}(t)\right)=\operatorname{cum}(\Delta J(0), \Delta J(t)),
\end{aligned}
$$

where the second equation can be justified by applying the Beppo Levi theorem both to the real and to the imaginary parts and considering that the convergence in (3.80) is essentially monotone. The last equation in (3.82) is the consequence of Theorem 6 . Thus, $S_{2, \Delta J}(\omega)$ is really the spectrum of the discrete time process $\Delta J(t)$. The first equation of (3.76) is the second equation of (3.79). The second equation of (3.76) arises from Proposition 23, since

$$
\begin{aligned}
S_{2, \Delta J}(\omega) & =\lim _{\lambda \rightarrow 0} S_{2, \Delta J_{\lambda}}(\omega) \\
& =\frac{1}{2 \pi} \cdot \frac{1}{1-H} c_{0} \sigma_{0}^{2}\left|\mathrm{e}^{i \omega}-1\right|^{2} \operatorname{Re}\left(\sum_{k=-\infty}^{\infty}\left(\left(\omega^{(k)}\right)^{-2} \int_{0}^{\infty} \mathrm{e}^{-i x \omega^{(k)}} x^{2(H-1)} \mathrm{d} x\right)\right) \\
& =\frac{1}{2 \pi} \cdot \frac{-\Gamma(2 H-1)}{1-H} c_{0} \sigma_{0}^{2}\left|\mathrm{e}^{i \omega}-1\right|^{2} \operatorname{Re}\left(\sum_{k=-\infty}^{\infty}\left(\left(i \omega^{(k)}\right)^{-1-2 H}\right)\right),
\end{aligned}
$$

where we also used formula (2.3.3.1) in [30]. At last, the third equation of (3.76) is just the last row of formula (3.79).

Remark 19. The spectrum of the $\Delta$ LISDLG process $\Delta J(t)$ is the same as that of the FGN, apart from some multiplicative constant.

Let us turn now to the bispectrum. 
Proposition 24. The bispectrum of the $\Delta \mathrm{ISDLG}$ process $\Delta J_{\lambda}(t)$ exists and it is

$$
\begin{aligned}
& S_{3, \Delta J_{\lambda}\left(\underline{\omega}_{(2)}\right)}=-i \prod_{j=1}^{3}\left(1-\mathrm{e}^{i \omega_{j}}\right) \sum_{k=-\infty}^{\infty} \sum_{\ell=-\infty}^{\infty}\left(\left(\omega_{1}^{(k)} \omega_{2}^{(\ell)} \omega_{3}^{(k+\ell)}\right)^{-1} S_{3, Y_{\lambda}}\left(\underline{\omega}_{(2)}^{(k, \ell)}\right)\right) \\
& =\left(\frac{1}{2 \pi}\right)^{2} \frac{6 i \mu_{0} \lambda^{3-2 H}}{1-H} \sigma_{0}^{4} \prod_{j=1}^{3}\left(1-\mathrm{e}^{i \omega_{j}}\right) \\
& \quad \times \operatorname{Im}\left\{\sum _ { k = - \infty } ^ { \infty } \sum _ { \ell = - \infty } ^ { \infty } \left[( \omega _ { 1 } ^ { ( k ) } \omega _ { 2 } ^ { ( \ell ) } \omega _ { 3 } ^ { ( k + \ell ) } ) ^ { - 2 } \underset { ( \underline { \omega } _ { ( 3 ) } ^ { ( k , \ell ) } ) } { \operatorname { s y m } } \left(\left(\omega_{1}^{(k)}\right)^{2} \mathrm{e}^{i \lambda \omega_{1}^{(k)}} \int_{\lambda}^{\infty} \mathrm{e}^{\left.\left.\left.-i x \omega_{1}^{(k)} x^{2(H-1)} \mathrm{d} x\right)\right]\right\}}\right.\right.\right.
\end{aligned}
$$

for $\left(\underline{\omega}_{(2)}\right) \in(0,2 \pi)^{2}$, such that $\omega_{3} \neq 0$, where $\omega_{3} \stackrel{\circ}{=}-\omega_{1}-\omega_{2}, \omega_{j}^{(k)} \stackrel{\circ}{=} \omega_{j}+2 k \pi, j=1,2, k \in \mathbb{Z}$, and

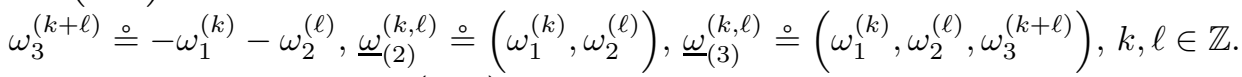

The bispectrum $S_{3, \Delta J_{\lambda}}\left(\underline{\omega}_{(2)}\right)$ has the extraordinary property that it is real-valued and positive.

Proof. Not only do the statements of this proposition and Proposition 23 correspond but the proofs are also very similar.

Let us calculate cum $\left(\Delta J_{\lambda}(0), \Delta J_{\lambda}(0), \Delta J_{\lambda}(0)\right)$. At first, we can change the order of the cumulant operator and the integrals by right of a similar reasoning as in (2.26) (see the explanation after (2.26)). Then applying Lemma 4 yields

$$
\begin{aligned}
\operatorname{cum}\left(\Delta J_{\lambda}(0), \Delta J_{\lambda}(0), \Delta J_{\lambda}(0)\right) & =\operatorname{cum}\left(\int_{0}^{1} Y_{\lambda}(s) \mathrm{d} s, \int_{0}^{1} Y_{\lambda}(s) \mathrm{d} s, \int_{0}^{1} Y_{\lambda}(s) \mathrm{d} s\right) \\
& =\int_{0}^{1} \int_{0}^{1} \int_{0}^{1} \operatorname{cum}\left(Y_{\lambda}\left(s_{0}\right), Y_{\lambda}\left(s_{1}\right), Y_{\lambda}\left(s_{2}\right)\right) \mathrm{d} s_{2} \mathrm{~d} s_{1} \mathrm{~d} s_{0} \\
& =2 \int_{0}^{1} \int_{0}^{1} \int_{0}^{1} \sigma_{0}^{4} \frac{\mu_{0} \lambda}{2(1-H)}\left(1+\frac{D_{\tau_{0}}(\underline{s})}{2 \lambda}\right)^{2(H-1)} \mathrm{d} \underline{s} \\
& =\frac{1}{1-H} \sigma_{0}^{4} \mu_{0} \lambda^{3-2 H} 3 ! \int_{0}^{1} \int_{s_{0}}^{1} \int_{s_{1}}^{1}\left(\lambda+s_{2}-s_{0}\right)^{2(H-1)} \mathrm{d} \underline{s} \\
& =\frac{3 \lambda^{3-2 H} \mu_{0} \sigma_{0}^{4}}{(1-H)(2 H-1) H}\left(\frac{2}{2 H+1}\left(\lambda^{2 H+1}-(\lambda+1)^{2 H+1}\right)+(\lambda+1)^{2 H}+\lambda^{2 H}\right) .
\end{aligned}
$$

We will need the resulting expression later, but presently we use only the fact that cum $\left(\Delta J_{\lambda}(0), \Delta J_{\lambda}(0)\right.$, $\left.\Delta J_{\lambda}(0)\right)$ is finite. 
Now let us calculate cum $\left(\Delta J_{\lambda}(0), \Delta J_{\lambda}\left(t_{1}\right), \Delta J_{\lambda}\left(t_{2}\right)\right)$ in general. We can change the order of the cumulant operator and the integrals as above, so by the stationarity, we have, that

$$
\begin{aligned}
\operatorname{cum}\left(\Delta J_{\lambda}(0), \Delta J_{\lambda}\left(t_{1}\right), \Delta J_{\lambda}\left(t_{2}\right)\right) & =\operatorname{cum}\left(\int_{0}^{1} Y_{\lambda}(s) \mathrm{d} s, \int_{t_{1}}^{t_{1}+1} Y_{\lambda}(s) \mathrm{d} s, \int_{t_{2}}^{t_{2}+1} Y_{\lambda}(s) \mathrm{d} s\right) \\
& =\int_{\substack{0 \\
\left(s_{0}\right)}}^{1} \int_{t_{1}}^{\left.t_{1}+s_{1}\right)} \int_{t_{2}}^{t_{1}} \int_{\left.s_{2}\right)}^{1 t_{2}+1}
\end{aligned}
$$

We know that $S_{3, Y_{\lambda}}\left(\underline{\omega}_{(2)}\right)$ is the bispectrum of the continuous time process $Y_{\lambda}(t)$, hence by continuing (3.84) we have

$$
\begin{aligned}
& \operatorname{cum}\left(\Delta J_{\lambda}(0), \Delta J_{\lambda}\left(t_{1}\right), \Delta J_{\lambda}\left(t_{2}\right)\right)=\int_{\substack{0 \\
\left(s_{0}\right)}}^{1} \int_{t_{1}}^{1} \int_{\substack{t_{2} \\
\left(s_{1}\right)\left(s_{2}\right)\left(\underline{\omega}_{(2)}\right)}}^{t_{1}+1} \int_{\mathbb{R}^{2}} \mathrm{e}^{i\left(\left(s_{1}-s_{0}\right) \omega_{1}+\left(s_{2}-s_{0}\right) \omega_{2}\right)} S_{3, Y_{\lambda}}\left(\underline{\omega}_{(2)}\right) \mathrm{d} \underline{\omega}(2) \mathrm{d} \underline{s}
\end{aligned}
$$

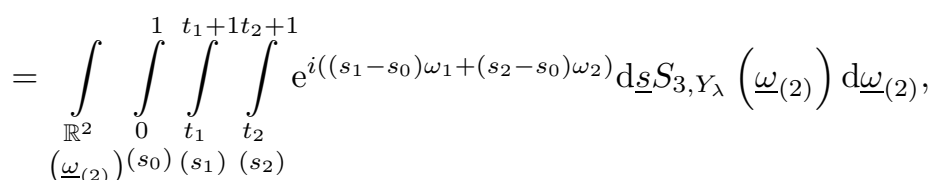

where the change of order of the integrals is the consequence of the integrability of $S_{3, Y_{\lambda}}\left(\underline{\omega}_{(2)}\right)$. Calculating the three inner integrals in the last row of (3.85), then cutting up the outer integral and performing the proper substitutions, we arrive at

$$
\begin{aligned}
& \operatorname{cum}\left(\Delta J_{\lambda}(0), \Delta J_{\lambda}\left(t_{1}\right), \Delta J_{\lambda}\left(t_{2}\right)\right)=-i \int_{\mathbb{R}^{2}} \mathrm{e}^{i\left(t_{1} \omega_{1}+t_{2} \omega_{2}\right)} \prod_{j=1}^{3}\left(1-\mathrm{e}^{i \omega_{j}}\right) \omega_{1}^{-1} \omega_{2}^{-1} \omega_{3}^{-1} S_{3, Y_{\lambda}}\left(\underline{\omega}_{(2)}\right) \mathrm{d} \underline{\omega}_{(2)} \\
& =\sum_{k=-\infty}^{\infty} \sum_{\ell=-\infty}^{\infty} \int_{[0,2 \pi]^{2}} \mathrm{e}^{i\left(t_{1} \omega_{1}+t_{2} \omega_{2}\right)}(-i) \prod_{j=1}^{3}\left(1-\mathrm{e}^{i \omega_{j}}\right)\left(\omega_{1}^{(k)} \omega_{2}^{(\ell)} \omega_{3}^{(k+\ell)}\right)^{-1} S_{3, Y_{\lambda}}\left(\underline{\omega}_{(2)}^{(k, \ell)}\right) \mathrm{d} \underline{\omega}_{(2)} .
\end{aligned}
$$

Remembering that $S_{3, Y_{\lambda}}\left(\underline{\omega}_{(2)}\right)$ is real-valued and positive, one can easily check that the function

$$
f\left(k, l, \underline{\omega}_{(2)}\right)=-i \prod_{j=1}^{3}\left(1-\mathrm{e}^{i \omega_{j}}\right)\left(\omega_{1}^{(k)} \omega_{2}^{(\ell)} \omega_{3}^{(k+\ell)}\right)^{-1} S_{3, Y_{\lambda}}\left(\underline{\omega}_{(2)}^{(k, \ell)}\right)
$$

is real and positive, too. Thus

$$
\begin{gathered}
\sum_{k=-\infty}^{\infty} \sum_{\ell=-\infty}^{\infty} \int_{[0,2 \pi]^{2}}\left|\mathrm{e}^{i\left(t_{1} \omega_{1}+t_{2} \omega_{2}\right)}(-i) \prod_{j=1}^{3}\left(1-\mathrm{e}^{i \omega_{j}}\right)\left(\omega_{1}^{(k)} \omega_{2}^{(\ell)} \omega_{3}^{(k+\ell)}\right)^{-1} S_{3, Y_{\lambda}}\left(\underline{\omega}_{(2)}^{(k, \ell)}\right)\right| \mathrm{d} \underline{\omega}_{(2)} \\
=\sum_{k=-\infty}^{\infty} \sum_{\ell=-\infty_{[0,2 \pi]^{2}}}^{\infty} f\left(k, l, \underline{\omega}_{(2)}\right) \mathrm{d} \underline{\omega}_{(2)}=\operatorname{cum}\left(\Delta J_{\lambda}(0), \Delta J_{\lambda}(0), \Delta J_{\lambda}(0)\right),
\end{gathered}
$$

where the last equation is (3.86) for $t_{1}=t_{2}=0$. The right-hand side of (3.88) is finite, see the beginning of this proof. Therefore by the Fubini theorem we can change the order of the double sum and the double 
integral in (3.86) to get

$$
\begin{aligned}
& \operatorname{cum}\left(\Delta J_{\lambda}(0), \Delta J_{\lambda}\left(t_{1}\right), \Delta J_{\lambda}\left(t_{2}\right)\right) \\
& =\int_{[0,2 \pi]^{2}} \mathrm{e}^{i\left(t_{1} \omega_{1}+t_{2} \omega_{2}\right)}(-i) \prod_{j=1}^{3}\left(1-\mathrm{e}^{i \omega_{j}}\right) \sum_{k=-\infty}^{\infty} \sum_{\ell=-\infty}^{\infty}\left[\left(\omega_{1}^{(k)} \omega_{2}^{(\ell)} \omega_{3}^{(k+\ell)}\right)^{-1} S_{3, Y_{\lambda}}\left(\underline{\omega}_{(2)}^{(k, \ell)}\right)\right] \mathrm{d} \underline{\omega}_{(2)} .
\end{aligned}
$$

Let us define the function

$$
S_{3, \Delta J_{\lambda}}\left(\underline{\omega}_{(2)}\right) \stackrel{\circ}{=}-i \prod_{j=1}^{3}\left(1-\mathrm{e}^{i \omega_{j}}\right) \sum_{k=-\infty}^{\infty} \sum_{\ell=-\infty}^{\infty}\left[\left(\omega_{1}^{(k)} \omega_{2}^{(\ell)} \omega_{3}^{(k+\ell)}\right)^{-1} S_{3, Y_{\lambda}}\left(\underline{\omega}_{(2)}^{(k, \ell)}\right)\right]
$$

for $\underline{\omega}_{(2)} \in(0,2 \pi)^{2}$ such that $\omega_{3} \neq 0$. We have just mentioned the integrability of $S_{3, \Delta J_{\lambda}}\left(\underline{\omega}_{(2)}\right)$, thus, the bispectrum of the discrete time process $\Delta J_{\lambda}(t)$ really exists and by $(3.89)$ it is $S_{3, \Delta J_{\lambda}}\left(\underline{\omega}_{(2)}\right)$. Moreover, by substituting the form $(3.56)$ of $S_{3, Y_{\lambda}}\left(\underline{\omega}_{(2)}\right)$ into $(3.90)$, we have

$$
\begin{aligned}
& S_{3, \Delta J_{\lambda}}\left(\underline{\omega}_{(2)}\right)=\left(\frac{1}{2 \pi}\right)^{2} \frac{6 i \mu_{0} \lambda^{3-2 H}}{1-H} \sigma_{0}^{4} \prod_{j=1}^{3}\left(1-\mathrm{e}^{i \omega_{j}}\right) \operatorname{Im}\left\{\sum_{k=-\infty}^{\infty} \sum_{\ell=-\infty}^{\infty}\right. \\
& \left.\left[\left(\omega_{1}^{(k)} \omega_{2}^{(\ell)} \omega_{3}^{(k+\ell)}\right)^{-2} \operatorname{sym}_{\left(\underline{\omega}_{(3)}^{(k, \ell)}\right)}\left(\left(\omega_{1}^{(k)}\right)^{2} \mathrm{e}^{i \lambda \omega_{1}^{(k)}} \int_{\lambda}^{\infty} \mathrm{e}^{-i x \omega_{1}^{(k)}} x^{2(H-1)} \mathrm{d} x\right)\right]\right\} .
\end{aligned}
$$

The fact that $S_{3, \Delta J_{\lambda}}\left(\underline{\omega}_{(2)}\right)$ is real-valued and positive, follows from its form (3.90) since function $f\left(k, l, \underline{\omega}_{(2)}\right)$ has the same properties.

Theorem 10. The bispectrum of the $\triangle L I S D L G$ process $\Delta J(t)$ exists and it is

$$
\begin{aligned}
& S_{3, \Delta J}\left(\underline{\omega}_{(2)}\right)=-i \prod_{j=1}^{3}\left(1-\mathrm{e}^{i \omega_{j}}\right) \sum_{k=-\infty}^{\infty} \sum_{\ell=-\infty}^{\infty}\left[\left(\omega_{1}^{(k)} \omega_{2}^{(\ell)} \omega_{3}^{(k+\ell)}\right)^{-1} S_{3, J^{\prime}}\left(\underline{\omega}_{(2)}^{(k, \ell)}\right)\right] \\
&=\left(\frac{1}{2 \pi}\right)^{2} \frac{-6 i \Gamma(2 H-1)}{1-H} c_{0} \sigma_{0}^{4} \\
& \times \prod_{j=1}^{3}\left(1-\mathrm{e}^{i \omega_{j}}\right) \operatorname{Im}\left\{\sum_{k=-\infty}^{\infty} \sum_{\ell=-\infty}^{\infty}\left[\left(\omega_{1}^{(k)} \omega_{2}^{(\ell)} \omega_{3}^{(k+\ell)}\right)^{-2} \operatorname{sym}_{\left(\underline{\omega}_{(3)}^{(k, \ell)}\right)}\left(\left(i \omega_{1}^{(k)}\right)^{3-2 H}\right)\right]\right\} \\
&=\frac{-3 i}{2 \pi \sin (H \pi) \Gamma(3-2 H)} c_{0} \sigma_{0}^{4} \prod_{j=1}^{3}\left(1-\mathrm{e}^{i \omega_{j}}\right) \sum_{k=-\infty}^{\infty} \sum_{\ell=-\infty}^{\infty}\left[\left(\omega_{1}^{(k)} \omega_{2}^{(\ell)} \omega_{3}^{(k+\ell)}\right)^{-1} \operatorname{sym}_{\left(\underline{\omega}_{(3)}^{(k, \ell)}\right)} \frac{\left.\omega_{1}^{(k)}\right|_{2} ^{(\ell)} \omega_{3}^{(k+\ell)}}{2(1-H)}\right]
\end{aligned}
$$

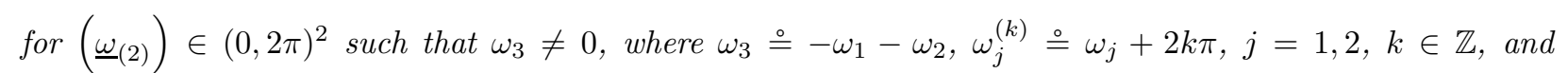

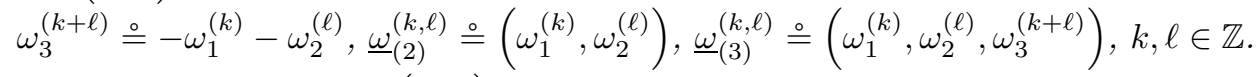

The bispectrum $S_{3, \Delta J}\left(\underline{\omega}_{(2)}\right)$ has the extraordinary property that it is real-valued moreover it is positive. 
Proof. By (3.57) and Condition 3,

$$
\begin{aligned}
S_{3, Y_{\lambda}}\left(\underline{\omega}_{(2)}\right) & =\mathrm{E}_{A} S_{3, R_{1,1}}\left(\underline{\omega}_{(2)}\right) \\
& =\left(\frac{1}{2 \pi}\right)^{2} \frac{12}{\Gamma(3-2 H)} \mu_{0} \lambda^{3-2 H} \sigma_{0}^{4} \int_{0}^{\infty} x^{2(1-H)} \mathrm{e}^{-\lambda x} \operatorname{sym}_{\left(\underline{\omega}_{(3)}\right)}\left(\frac{1}{x} \cdot \frac{1}{-i \omega_{1}+x} \cdot \frac{1}{i \omega_{2}+x}\right) \mathrm{d} x
\end{aligned}
$$

hence by (3.59) and Condition 7,

$$
\begin{aligned}
S_{3, J^{\prime}}\left(\underline{\omega}_{(2)}\right) & =\lim _{\lambda \rightarrow 0} S_{3, Y_{\lambda}}\left(\underline{\omega}_{(2)}\right) \\
& =\left(\frac{1}{2 \pi}\right)^{2} \frac{12}{\Gamma(3-2 H)} c_{0} \sigma_{0}^{4} \int_{0}^{\infty} x^{2(1-H)} \underset{\left(\underline{\omega}_{(3)}\right)}{\operatorname{sym}}\left(\frac{1}{x} \cdot \frac{1}{-i \omega_{1}+x} \cdot \frac{1}{i \omega_{2}+x}\right) \mathrm{d} x .
\end{aligned}
$$

Moreover, the convergence

$$
\int_{0}^{\infty} x^{2(1-H)} \mathrm{e}^{-\lambda x} \operatorname{sym}_{\left(\underline{\omega}_{(3)}\right)}\left(\frac{1}{x} \cdot \frac{1}{-i \omega_{1}+x} \cdot \frac{1}{i \omega_{2}+x}\right) \mathrm{d} x \underset{\lambda \rightarrow 0}{\longrightarrow} \int_{0}^{\infty} x^{2(1-H)} \operatorname{sym}_{\left(\underline{\omega}_{(3)}\right)}\left(\frac{1}{x} \cdot \frac{1}{-i \omega_{1}+x} \cdot \frac{1}{i \omega_{2}+x}\right) \mathrm{d} x
$$

is monotone, that is, for every $\omega_{1}, \omega_{2} \in \mathbb{R}$ such that $\omega_{1} \omega_{2} \omega_{3} \neq 0$, the left-hand side of (3.93) increases as $\lambda$ decreases. The motivation is the nonnegativity of $S_{3, R_{1,1}}\left(\underline{\omega}_{(2)}\right)$ and thus of the symmetrized term in (3.93). Observe also that the convergence in (3.92) is essentially monotone.

At this point we anticipate that for each $(k, \ell) \in \mathbb{Z}^{2}$ the term $-i \prod_{j=1}^{3}\left(1-\mathrm{e}^{i \omega_{j}}\right)\left(\omega_{1}^{(k)} \omega_{2}^{(\ell)} \omega_{3}^{(k+\ell)}\right)^{-1}$ is real-valued and positive, as it is easy to see.

By Proposition 24 and formulae (3.60) and (3.61), we have

$$
\begin{aligned}
& \lim _{\lambda \rightarrow 0} S_{3, \Delta J_{\lambda}}\left(\underline{\omega}_{(2)}\right)=-i \prod_{j=1}^{3}\left(1-\mathrm{e}^{i \omega_{j}}\right) \lim _{\lambda \rightarrow 0} \sum_{k=-\infty}^{\infty} \sum_{\ell=-\infty}^{\infty}\left[\left(\omega_{1}^{(k)} \omega_{2}^{(\ell)} \omega_{3}^{(k+\ell)}\right)^{-1} S_{3, Y_{\lambda}}\left(\underline{\omega}_{(2)}^{(k, \ell)}\right)\right] \\
& =-i \prod_{j=1}^{3}\left(1-\mathrm{e}^{i \omega_{j}}\right) \sum_{k=-\infty}^{\infty} \sum_{\ell=-\infty}^{\infty}\left[\left(\omega_{1}^{(k)} \omega_{2}^{(\ell)} \omega_{3}^{(k+\ell)}\right)^{-1} S_{3, J^{\prime}}\left(\underline{\omega}_{(2)}^{(k, \ell)}\right)\right] \\
& =\left(\frac{1}{2 \pi}\right)^{2} \frac{-6 i c_{0} \sigma_{0}^{4} \Gamma(2 H-1)}{1-H} \\
& \times \prod_{j=1}^{3}\left(1-\mathrm{e}^{i \omega_{j}}\right) \operatorname{Im}\left\{\sum_{k=-\infty}^{\infty} \sum_{\ell=-\infty}^{\infty}\left[\left(\omega_{1}^{(k)} \omega_{2}^{(\ell)} \omega_{3}^{(k+\ell)}\right)^{-2} \underset{\substack{\left(\underline{\omega}_{(3)}^{(k, \ell)}\right) \\
\operatorname{sym}}}{ }\left(\left(i \omega_{1}^{(k)}\right)^{3-2 H}\right)\right]\right\} \\
& =\frac{-3 i}{2 \pi \sin (H \pi) \Gamma(3-2 H)} c_{0} \sigma_{0}^{4} \prod_{j=1}^{3}\left(1-\mathrm{e}^{i \omega_{j}}\right) \sum_{k=-\infty}^{\infty} \sum_{\ell=-\infty}^{\infty}\left[\left(\omega_{1}^{(k)} \omega_{2}^{(\ell)} \omega_{3}^{(k+\ell)}\right)^{-1} \operatorname{sym}_{\left(\underline{\omega}_{(3)}^{(k, \ell)}\right)} \frac{\left|\omega_{1}^{(k)}\right|^{2(1-H)}}{\omega_{2}^{(\ell)} \omega_{3}^{(k+\ell)}}\right] \text {, }
\end{aligned}
$$

which is finite for every $\underline{\omega}_{(2)} \in(0,2 \pi)^{2}$ such that $\omega_{1} \omega_{2} \omega_{3} \neq 0$, because it is even integrable on $[0,2 \pi]^{2}$, as we will see immediately. In (3.94) changing the order of the limit and the infinite sum can be justified by the Beppo Levi theorem and the fact that the convergence (3.92) is essentially monotone. Therefore let us define the function

$$
S_{3, \Delta J}\left(\underline{\omega}_{(2)}\right) \stackrel{\circ}{=} \lim _{\lambda \rightarrow 0} S_{3, \Delta J_{\lambda}}\left(\underline{\omega}_{(2)}\right)
$$


for every $\underline{\omega}_{(2)} \in(0,2 \pi)^{2}$ such that $\omega_{1} \omega_{2} \omega_{3} \neq 0$. Then $S_{3, \Delta J}\left(\underline{\omega}_{(2)}\right)$ is integrable since by the Fatou lemma we have

$$
\begin{gathered}
\int_{[0,2 \pi]^{2}}\left|S_{3, \Delta J}\left(\underline{\omega}_{(2)}\right)\right| \mathrm{d} \underline{\omega}_{(2)}=\int_{[0,2 \pi]^{2}} \underline{\lim }\left|S_{3, \Delta J_{\lambda}}\left(\underline{\omega}_{(2)}\right)\right| \mathrm{d} \underline{\omega}_{(2)} \\
\leqslant \frac{\lim }{\lambda \rightarrow 0} \int_{[0,2 \pi]^{2}}\left|S_{3, \Delta J_{\lambda}}\left(\underline{\omega}_{(2)}\right)\right| \mathrm{d} \underline{\omega}_{(2)}=\underline{\lim }_{\lambda \rightarrow 0} \operatorname{cum}\left(\Delta J_{\lambda}(0), \Delta J_{\lambda}(0), \Delta J_{\lambda}(0)\right) \\
=\underline{\lim }\left[\mu_{0} \lambda^{3-2 H} \sigma_{0}^{4} \frac{3}{(1-H)(2 H-1) H}\left(\frac{2}{2 H+1}\left(\lambda^{2 H+1}-(\lambda+1)^{2 H+1}\right)+(\lambda+1)^{2 H}+\lambda^{2 H}\right)\right] \\
=c_{0} \sigma_{0}^{4} \frac{3}{(1-H) H(2 H+1)}<\infty .
\end{gathered}
$$

The third equation in (3.96) is the consequence of formula (3.83). Moreover,

$$
\begin{gathered}
\int_{[0,2 \pi]^{2}} \mathrm{e}^{i\left(t_{1} \omega_{1}+t_{2} \omega_{2}\right)} S_{3, \Delta J}\left(\underline{\omega}_{(2)}\right) \mathrm{d} \underline{\omega}_{(2)}=\int_{[0,2 \pi]^{2}} \mathrm{e}^{i\left(t_{1} \omega_{1}+t_{2} \omega_{2}\right)} \lim _{\lambda \rightarrow 0} S_{3, \Delta J_{\lambda}}\left(\underline{\omega}_{(2)}\right) \mathrm{d} \underline{\omega}_{(2)} \\
=\lim _{\lambda \rightarrow 0} \int_{[0,2 \pi]^{2}} \mathrm{e}^{i\left(t_{1} \omega_{1}+t_{2} \omega_{2}\right)} S_{3, \Delta J_{\lambda}}\left(\underline{\omega}_{(2)}\right) \mathrm{d} \underline{\omega}_{(2)}=\lim _{\lambda \rightarrow 0} \operatorname{cum}\left(\Delta J_{\lambda}(0), \Delta J_{\lambda}\left(t_{1}\right), \Delta J_{\lambda}\left(t_{2}\right)\right) \\
=\operatorname{cum}\left(\Delta J(0), \Delta J\left(t_{1}\right), \Delta J\left(t_{2}\right)\right),
\end{gathered}
$$

where the second equation can be justified by applying the Beppo Levi theorem both to the real and to the imaginary parts, and considering that the convergence in (3.95) is essentially monotone. The last equation in (3.97) is the consequence of Theorem 6. Thus $S_{3, \Delta J}\left(\underline{\omega}_{(2)}\right)$ is really the bispectrum of the $\Delta$ LISDLG process $\Delta J(t)$. Equation (3.91) follows from (3.95) and (3.94).

The fact that $S_{3, \Delta J}\left(\underline{\omega}_{(2)}\right)$ is real-valued and positive, is the consequence of the fact that the same property holds for each of the terms $S_{3, J^{\prime}}\left(\underline{\omega}_{(2)}^{(k, \ell)}\right)$ and $(-i) \prod_{j=1}^{3}\left(1-\mathrm{e}^{i \omega_{j}}\right)\left(\omega_{1}^{(k)} \omega_{2}^{(\ell)} \omega_{3}^{(k+\ell)}\right)^{-1}$ on the right-hand side of the first row of (3.91).

Remark 20. The bispectrum $S_{3, \Delta J}\left(\underline{\omega}_{(2)}\right)$ behaves around zero as

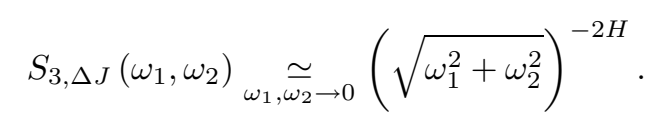

Remark 21. The property of the bispectrum $S_{3, \Delta J}\left(\underline{\omega}_{(2)}\right)$ that it is real-valued is equivalent to the property of the cumulants that

$$
\operatorname{cum}\left(\Delta J(0), \Delta J\left(t_{1}\right), \Delta J\left(t_{2}\right)\right)=\operatorname{cum}\left(\Delta J(0), \Delta J\left(t_{2}-t_{1}\right), \Delta J\left(t_{2}\right)\right),
$$

$t_{1}, t_{2} \in \mathbb{Z}$, see also Remark 13 .

Proof. See the proof of Remark 17. 
On the other hand, the generalization of (3.98) for cumulants of order $m \geqslant 3$ also holds, that is to say, we have

\section{Remark 22.}

$$
\operatorname{cum}\left(\Delta J(0), \Delta J\left(t_{1}\right), \ldots, \Delta J\left(t_{m-1}\right)\right)=\operatorname{cum}\left(\Delta J(0), \Delta J\left(t_{m-1}-t_{m-2}\right), \ldots, \Delta J\left(t_{m-1}\right)\right)
$$

for all $m \geq 3$ and $t_{1}, \ldots, t_{m-1} \in \mathbb{Z}$.

Proof. Similar to that of Remark 18.

\subsection{Nonlinearity of the $\Delta$ ISDLG and $\Delta$ LISDLG processes}

The notion of nonlinearity has several meanings in time series analysis. The most frequent is the following. Let $X(t), t \in \mathbb{Z}$, be a discrete time $L^{2}$-stationary process with zero mean. It is called a linear process, if it has an $L^{2}$-convergent moving average representation with independent and identically distributed (i.i.d.) input variables, i.e.

$$
X(t)=\sum_{k=-\infty}^{\infty} c(k) Z(t-k)
$$

where

$$
\sum_{k=-\infty}^{\infty} c(k)^{2}<\infty
$$

and the input random variables $Z(t), t \in \mathbb{Z}$, are i.i.d., $\mathrm{E} Z(t)=0$ and $\mathrm{E} Z(t)^{2}<\infty$. Otherwise process $X(t)$ is said to be nonlinear.

Let us consider a third order stationary process $X(t)$, linear in the above sense and having bispectrum (in the sense of the paragraph before Prop. 23). By the Marcinkiewicz-Zygmund inequality (see e.g. [35]) the input random variables $Z(t)$ also have finite third order moment. Therefore the bispectrum of process $X(t)$ has the form

$$
S_{3, X}\left(\underline{\omega}_{(2)}\right)=\frac{\operatorname{cum}_{3}(Z(0))}{(2 \pi)^{2}} \varphi\left(\omega_{1}\right) \varphi\left(\omega_{2}\right) \varphi\left(-\omega_{1}-\omega_{2}\right),
$$

where

$$
\varphi(\omega)=\sum_{k=-\infty}^{\infty} c(k) \mathrm{e}^{-i k \omega}
$$

$\omega \in[0,2 \pi)$, is the frequency domain transfer function of process $X(t)$ with moving average representation (3.99), see [15], Lemma 7. However, if $X(t)$ is also linearly regular and long-range dependent then such a bispectrum cannot be real. Summing up, it holds true the following lemma, which is a somewhat sharpened version of the theorem in [15].

Lemma 6. If a discrete time process with zero mean is linearly regular, long-range dependent and it has real-valued bispectrum, then it is nonlinear.

The discrete time processes $\Delta J_{\lambda}(t)$ and $\Delta J(t)$ are linearly regular, because the spectrum of each process obviously satisfies the Kolmogorov-Szegö condition. They are long-range dependent as well. Taking into account that they have real-valued bispectra, see Propositions 24 and 10, we can derive the following theorem.

Theorem 11. Both the $\triangle I S D L G$ process $\Delta J_{\lambda}(t)$ and the $\Delta L I S D L G$ process $\Delta J(t)$ are nonlinear. 


\subsection{Relation with [1], a similar result of Barndorff-Nielsen}

A paper [1] by Barndorff-Nielsen also deals with the superposition of OU processes and the limit of its integral process. The buildup of its model is very similar to ours, but the terminology and the initial processes, i.e. the processes to be superposed are totally different. In effect the treatment in [1] is somewhat more general than ours, so we will test it only from our point of view.

Our notation is unlike that of the paper under review in order to make the correspondence of the two models evident. Now, in [1] there is defined a family $Y(t, \cdot), t \in \mathbb{R}$, of random measures $Y(t, B), B \in \mathcal{B}\left(\mathbb{R}_{+}\right)$, on $\mathbb{R}_{+}$, in terms of integrating with respect to an independently scattered random measure $Z(\mathrm{~d} t, \mathrm{~d} x)$ on the set $\mathbb{R} \times \mathbb{R}_{+}$, i.e.

$$
Y(t, B) \stackrel{\circ}{=} \int_{B} \mathrm{e}^{-x t} \int_{-\infty}^{x t} \mathrm{e}^{s} Z(\mathrm{~d} s, \mathrm{~d} x)
$$

and the process proper is

$$
Y(t) \stackrel{=}{=}\left(t, \mathbb{R}_{+}\right)
$$

From (3.101) and (3.102) one has

$$
\mathrm{d} Y(t)=\int_{\mathbb{R}_{+}}(-x Y(t, \mathrm{~d} x) \mathrm{d} t+Z(x \mathrm{~d} s, \mathrm{~d} x)),
$$

showing that $Y(t)$ is really a superposition of perhaps infinitesimally determined OU processes.

Let the generalized Lévy measure $Q(A, \mathrm{~d} t)$ in the Lévy-Khinchin representation

$$
\log \left(\varphi_{Z(A)}(u)\right)=\log \left(\mathrm{Ee}^{i Z(A) u}\right)=\int_{\mathbb{R}}\left(\mathrm{e}^{i u t}-1-i u \tau(t)\right) Q(A, \mathrm{~d} t)
$$

where $A \in \mathcal{B}\left(\mathbb{R} \times \mathbb{R}_{+}\right)$, factorize as

$$
Q(A, \mathrm{~d} t)=M(A) V(\mathrm{~d} t)
$$

for some measure $M$ on $\mathbb{R} \times \mathbb{R}_{+}$and some Lévy measure $V$ on $\mathbb{R}$. Moreover let $M$ factorize as

$$
M(\mathrm{~d} s, \mathrm{~d} x)=\mathrm{d} s N(\mathrm{~d} x),
$$

for the Lebesgue measure $\mathrm{d} s$ on $\mathbb{R}$ and a probability measure $N$ on $\mathbb{R}_{+}$. The paper under discussion, similarly to our paper, uses the $\Gamma$ distribution, but with a different shape parameter, namely,

$$
N \stackrel{\circ}{=}(2(1-H), 1 / \lambda)
$$

where the parameters are $1 / 2<H<1$ and $0<\lambda$. We denote the dependence on $\lambda$ as we did in the case of the SDLG process $Y(t)=Y_{\lambda}(t)$.

The joint cumulants of the superposition process $Y(t)$, if they exist, are also given in the formula (3.5) by [1], showing that

$$
\operatorname{cum}\left(Y\left(t_{1}\right), \ldots Y\left(t_{m}\right)\right)=\int_{\mathbb{R}_{+}} \operatorname{cum}\left(Y^{(x)}\left(t_{1}\right), \ldots Y^{(x)}\left(t_{m}\right)\right) N(\mathrm{~d} x)
$$


i.e. the mixture of the corresponding cumulants of the component processes $Y^{(x)}(t), x \in \mathbb{R}_{+}$, by the weight distribution $N$. For each $x \in \mathbb{R}_{+}$, the component process $Y^{(x)}(t)$ in (3.106) is the OU process defined by the Langevin equation

$$
\mathrm{d} Y^{(x)}(t)=-x Y^{(x)}(t) \mathrm{d} t+Z^{(x)}(x \mathrm{~d} t)
$$

where the independently scattered random measure $Z^{(x)}(\mathrm{d} t) \stackrel{\ominus}{=}(\mathrm{d} t, \mathrm{~d} x)$, choosing the measure $N$ in $(3.104)$ to be

$$
N=P_{x}
$$

the atomic measure concentrated at point $x$. That is, $Y^{(x)}(t)$ and $Z^{(x)}(\mathrm{d} t)$ are the same as $Y(t)$ and $Z(\mathrm{~d} t, \mathrm{~d} x)$, respectively, except that $N$ is different, being defined by (3.107) and (3.105). We will also denote the dependence on $\lambda$ as, e.g., $Y_{\lambda}^{(x)}(t)$.

The integrated process

$$
J_{\lambda}(t) \stackrel{\circ}{t} \int_{0}^{t} Y_{\lambda}(s) \mathrm{d} s
$$

is also defined, and a theorem is stated and proved about the convergence as $\lambda \rightarrow 0$, of the family of processes $J_{\lambda}(t), \lambda>0$. More precisely, assume that the Lévy measure $V$ in (3.103) also depends on $\lambda$, viz.

$$
V_{\lambda}(\mathrm{d} t)=\lambda^{2(H-1)} V(\mathrm{~d} t)
$$

Under condition (3.108) and some other condition imposed on the logarithmic characteristic function of the stationary process $Y_{1}(t)$, the finite-dimensional distributions of $J_{\lambda}(t)$ converge weakly to those of some process $J(t)$, i.e.

$$
J_{\lambda} \underset{\lambda \rightarrow 0}{\stackrel{w}{\longrightarrow}} J
$$

in the finite-dimensional sense, see Theorem 6.1 of [1]. Of course, process $J(t)$ has stationary increments.

Now, assuming that the Lévy measure $V$ is such that $J(1)$ has a finite second order moment, we have

$$
\operatorname{cov}\left(J\left(t_{1}\right), J\left(t_{2}\right)\right)=\text { const. }\left(\left|t_{1}\right|^{2 H}+\left|t_{2}\right|^{2 H}-\left|t_{2}-t_{1}\right|^{2 H}\right)
$$

(see Ex. 6.2 in [1]), the same as the covariance of the LISDLG process $J(t)$ (and of the FBM). More generally, there is a close resemblance between the two processes $J(t)$. In particular, as long as the cumulants exist, for both of them

$$
\operatorname{cum}_{m}(J(t))=k(m) t^{m+2(H-1)}
$$

holds for $m \geqslant 2$, where $k(m), m \geqslant 2$, are some positive constants not depending on $t$, see Theorem 7 in this paper and Example 6.3 in [1]. (Naturally, constants $k(m)$ are different for the two processes $J(t)$.) Thus, both processes $J(t)$ are multifractal with the same exponents.

It follows from (3.109) that the generalized second order spectra appertaining to the two processes are the same, namely, $S_{2, J^{\prime}}(\omega)=$ const. $|\omega|^{1-2 H}$, see Proposition 20 . We state this in the following proposition:

Proposition 25. The generalized spectrum appertaining to process $J(t)$ of [1], i.e.

$$
S_{2, J^{\prime}}(\omega) \stackrel{\circ}{=} \lim _{\lambda \rightarrow 0} S_{2, Y_{\lambda}}(\omega),
$$

is given by

$$
S_{2, J^{\prime}}(\omega)=\text { const. }|\omega|^{1-2 H} \text {. }
$$


Thus it is the same as the generalized spectrum appertaining to the LISDLG process $J(t)$.

After all, the two processes are significantly different. The discrepancy shows itself by means of the different properties of the generalized bispectra. That is to say, the generalized bispectrum appertaining to the LISDLG process $J(t)$ is real-valued, see Proposition 22, in contrast to the generalized bispectrum appertaining to process $J(t)$ of [1].

Proposition 26. The generalized bispectrum appertaining to process $J(t)$ of [1], i.e.

$$
S_{3, J^{\prime}}\left(\underline{\omega}_{(2)}\right) \stackrel{\circ}{=} \lim _{\lambda \rightarrow 0} S_{3, Y_{\lambda}}\left(\underline{\omega}_{(2)}\right)
$$

if it exists at all, cannot be real-valued apart from the case when it is zero.

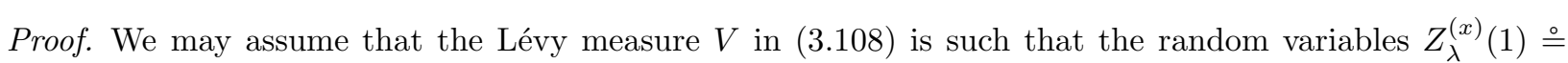
$Z_{\lambda}^{(x)}((0,1]), x \in \mathbb{R}_{+}, \lambda>0$, have finite third order cumulants, because in the opposite case the bispectra of processes $Y_{\lambda}^{(x)}(t), x \in \mathbb{R}_{+}, \lambda>0$, do not exist, thus, neither do the bispectra of processes $Y_{\lambda}(t), \lambda>0$, so the generalized bispectrum appertaining to process $J(t)$ is not defined either.

Since for each $x \in \mathbb{R}_{+}$process $Y_{\lambda}^{(x)}(t)$ is an OU process with a zero expectation and a finite second order moment, its spectral representation is

$$
Y_{\lambda}^{(x)}(t)=\int_{\mathbb{R}} \mathrm{e}^{i t \omega} \frac{1}{i \omega+x} W_{\lambda}^{(x)}(\mathrm{d} \omega)
$$

with some stochastic spectral measure $W_{\lambda}^{(x)}(\mathrm{d} \omega)$ on $\mathbb{R}$. Since $Y_{\lambda}^{(x)}(t)$ is a linear process with an integrable transfer function in its moving average representation (being an OU process), its bispectrum is the three-term product

$$
\begin{aligned}
S_{3, Y_{\lambda}^{(x)}}\left(\underline{\omega}_{(2)}\right) & =\left(\frac{1}{2 \pi}\right)^{2} c_{3}(x, \lambda) \frac{1}{i \omega_{1}+x} \cdot \frac{1}{i \omega_{2}+x} \cdot \frac{1}{-i\left(\omega_{1}+\omega_{2}\right)+x} \\
& =\left(\frac{1}{2 \pi}\right)^{2} c_{3}(x, \lambda) \frac{x\left(x^{2}+\omega_{1}^{2}+\omega_{2}^{2}+\omega_{1} \omega_{2}\right)-i\left(\omega_{1}+\omega_{2}\right) \omega_{1} \omega_{2}}{x^{2}\left(x^{2}+\omega_{1}^{2}+\omega_{2}^{2}+\omega_{1} \omega_{2}\right)^{2}+\left(\omega_{1}+\omega_{2}\right)^{2} \omega_{1}^{2} \omega_{2}^{2}}
\end{aligned}
$$

(see Ex. 3 in [3]), where

$$
c_{3}(x, \lambda)=x \operatorname{cum}_{3}\left(Z_{\lambda}^{(x)}(1)\right)=x \lambda^{2(H-1)} \operatorname{cum}_{3}\left(Z_{1}^{(x)}(1)\right)=x \lambda^{2(H-1)} \widetilde{c_{3}},
$$

and $\widetilde{c_{3}} \in \mathbb{R}$ is a constant depending neither on $x$ nor on $\lambda$.

On the other hand, because of (3.106) the bispectrum of $Y_{\lambda}(t)$ is the mixture of the bispectra of $Y_{\lambda}^{(x)}(t)$, i.e.

$$
S_{3, Y_{\lambda}}\left(\underline{\omega}_{(2)}\right)=\int_{\mathbb{R}_{+}} S_{3, Y_{\lambda}^{(x)}}\left(\underline{\omega}_{(2)}\right) N(\mathrm{~d} x)
$$

where the distribution $N$ is the $\Gamma$ distribution given by (3.105). Hence by substituting the results of (3.110) and (3.111) into (3.112), one has

$$
S_{3, Y_{\lambda}}\left(\underline{\omega}_{(2)}\right)=\left(\frac{1}{2 \pi}\right)^{2} \lambda^{2(H-1)} \widetilde{c_{3}} \int_{\mathbb{R}_{+}} x \frac{x\left(x^{2}+\omega_{1}^{2}+\omega_{2}^{2}+\omega_{1} \omega_{2}\right)-i\left(\omega_{1}+\omega_{2}\right) \omega_{1} \omega_{2}}{x^{2}\left(x^{2}+\omega_{1}^{2}+\omega_{2}^{2}+\omega_{1} \omega_{2}\right)^{2}+\left(\omega_{1}+\omega_{2}\right)^{2} \omega_{1}^{2} \omega_{2}^{2}} N(\mathrm{~d} x),
$$


and therefore

$$
\begin{aligned}
S_{3, J^{\prime}}(\underline{\underline{\omega}}(2))= & \frac{\widetilde{c_{3}}}{(2 \pi)^{2}} \lim _{\lambda \rightarrow 0}\left(\lambda^{2(H-1)} \frac{\lambda^{2(1-H)}}{\Gamma(2(1-H))} \int_{\mathbb{R}_{+}} x^{2(1-H)} \mathrm{e}^{-\lambda x}\right. \\
& \left.\times \frac{x\left(x^{2}+\omega_{1}^{2}+\omega_{2}^{2}+\omega_{1} \omega_{2}\right)-i\left(\omega_{1}+\omega_{2}\right) \omega_{1} \omega_{2}}{x^{2}\left(x^{2}+\omega_{1}^{2}+\omega_{2}^{2}+\omega_{1} \omega_{2}\right)^{2}+\left(\omega_{1}+\omega_{2}\right)^{2} \omega_{1}^{2} \omega_{2}^{2}} \mathrm{~d} x\right) \\
= & \widetilde{c_{3}} \frac{1}{(2 \pi)^{2} \Gamma(2(1-H))} \int_{\mathbb{R}_{+}} \frac{x^{3-2 H}\left(x^{2}+\omega_{1}^{2}+\omega_{2}^{2}+\omega_{1} \omega_{2}\right)}{x^{2}\left(x^{2}+\omega_{1}^{2}+\omega_{2}^{2}+\omega_{1} \omega_{2}\right)^{2}+\left(\omega_{1}+\omega_{2}\right)^{2} \omega_{1}^{2} \omega_{2}^{2}} \mathrm{~d} x \\
& -i \widetilde{c_{3}} \frac{\left(\omega_{1}+\omega_{2}\right) \omega_{1} \omega_{2}}{(2 \pi)^{2} \Gamma(2(1-H))} \int_{\mathbb{R}_{+}} \frac{x^{2(1-H)}}{x^{2}\left(x^{2}+\omega_{1}^{2}+\omega_{2}^{2}+\omega_{1} \omega_{2}\right)^{2}+\left(\omega_{1}+\omega_{2}\right)^{2} \omega_{1}^{2} \omega_{2}^{2}} \mathrm{~d} x .
\end{aligned}
$$

Now, if the generalized bispectrum $S_{3, J^{\prime}}\left(\underline{\omega}_{(2)}\right)$ is real, then $\widetilde{c_{3}}=0$, thus $S_{3, J^{\prime}}\left(\underline{\omega}_{(2)}\right)=0$.

\section{Application to COMputer network trafFic DAta}

The earlier paper [41] raised the problem of finding a stochastic process all of whose increment processes have the following properties:

- stationarity;

- LRD;

- real-valued bispectrum;

- nonlinearity;

- $\Gamma$ one-dimensional distribution (or nearly $\Gamma$ in some sense).

Multifractal property was not positively required but was alluded to. The motivation was that the computer network traffic data set investigated by the authors of that paper mainly from the point of view of spectralbispectral analysis, showed basically the above properties. The $\Delta$ LISDLG process does have these properties. Here we wish to demonstrate, in particular graphically, that a fair fit can be found between the network traffic data centralized to zero and the $\triangle$ LISDLG process.

We analyzed the time series of aggregated 155 Mbps SUNET ATM WAN (Swedish University Network, Asynchronous Transfer Mode, Wide Area Network) traffic ${ }^{3}$ on the $\approx 0.1 \mathrm{~s}$ (approximately $0.1 \mathrm{~s}$ ) level. That is to say, the source data set consisting of 425 million cell arrivals (slots) was divided into blocks of size 8000 slots, approximately this is the amount of slots piping in $0.1 \mathrm{~s}$. Then for each block the block sum was calculated. Thus the aggregated data set consists of about 425 million/8000 53000 measurements. It will be called $\approx 0.1 \mathrm{~s}$ level ATM data, or briefly ATM data. We will also use the data set called $\approx 1 \mathrm{~s}$ level ATM data arising by aggregating the $\approx 0.1 \mathrm{~s}$ level ATM data in each block of size 10 .

\subsection{The dilative stability and parameter estimation}

At first we demonstrate one-dimensional dilative stability, a particular manifestation of the multifractal property, see Proposition 17. Figure 1 shows in logarithmic scale of how the estimated cumulants of the aggregated data depend on the level of aggregation. The aggregation is meant in the sense of Corollary 4 , i.e. it means averaging. The orders of the cumulants is in the range $\{2, \ldots, 6\}$. If the data came from a

\footnotetext{
${ }^{3}$ The reported ATM WAN measurements were carried out by the CARAT laboratory of Telia Research in Sweden. We would like to thank them and Sándor Molnár in High Speed Networks Lab., Dept. of Telecommunications \& Telematics, Technical University of Budapest, for providing the data. Special thanks go to our colleague Zoltán Gál in Center for Informatics and Computing, University of Debrecen, for joint work.
} 
$\triangle$ LISDLG process, then by taking the logarithm in (3.34) for each fixed order $m \in\{2, \ldots, 6\}$ the relationship would be

$$
\log \operatorname{cum}_{m}\left(\Delta J^{\left(n_{i}\right)}\right)=\log k_{1}(m)+2(H-1) \log n_{i}
$$

where the levels of time-aggregation are $n_{i}, i=1, \ldots, r$. In Figure $1, r=25$ and $n_{i}$ ranges from $n_{1}=4$ to $n_{25}=223$ such that $\log n_{i}, i=1, \ldots, 25$, is an approximately equidistant sequence. For each cumulant order $m$, (4.1) means that the dependence is linear and the lines must be parallel, each with slope $2(H-1)$. This is so in Figure 1, where the five linear point sets, corresponding upwards to $m=2, \ldots, 6$, confirm the $\Delta$ LISDLG model. The common slope parameter $2(H-1)$ and constants $\log k_{1}(m), m=2, \ldots, 6$, were estimated by weighted linear regression from the linear model with equations $(4.1), i=1, \ldots, r, m=2, \ldots, 6$, where the weights are the inverse of the standard deviations of the logarithm of the estimated cumulants. The LRD parameter $H$ estimated in this way came out to be ${ }^{4}$

$$
\widehat{H}=0.9156 \text {. }
$$

In possession of the estimated $H$, the constants depending on $m$ and $H$ in (3.34), including the integral term could be obtained. By the way, the latter term was computed by Monte-Carlo method. Then constants $c_{0}$ and $\sigma_{0}^{2}$ were estimated, also by linear regression, to be $\widehat{c_{0}}=38.3156$ and $\widehat{\sigma_{0}^{2}}=29.0833$.

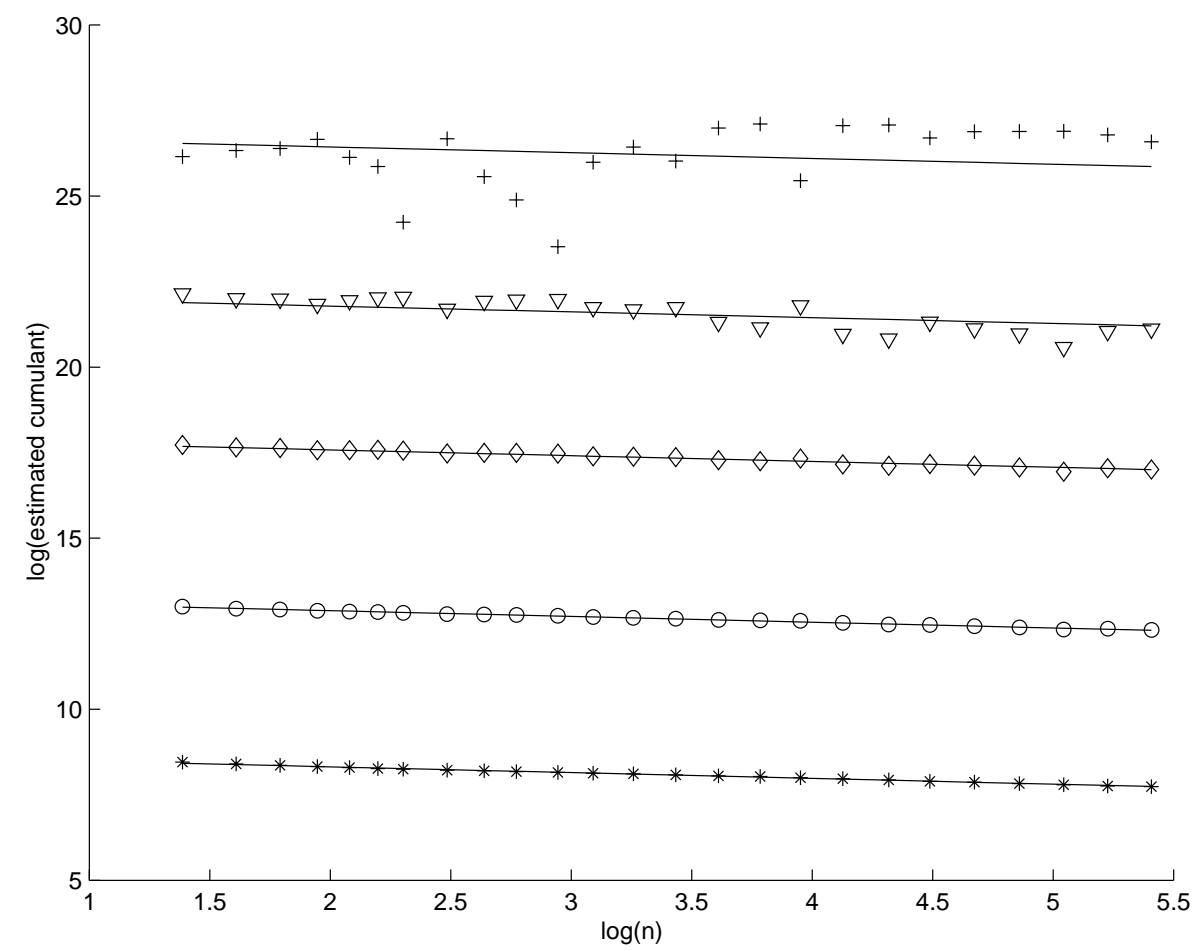

Figure 1. One-dimensional dilative stability illustrated by cumulants. The discrete points corresponds to the data. Points plotted with the same signs have coordinates $\left(\log n_{i}, \log \widehat{\operatorname{cum}_{m}}\left(\Delta J^{\left(n_{i}\right)}\right)\right), i=1, \ldots, 25$. The orders of the cumulants are $m=2, \ldots, 6$, upwards. The continuous lines fitted by weighted linear regression have the common slope $2(\widehat{H}-1)$, where $\widehat{H}=0.9156$.

\footnotetext{
${ }^{4}$ Examination of statistical methods is not the aim of this paper. However, it should be noticed that it is not hard to see that stationarity and the law of large numbers ensure the consistency of the cumulant estimates and the characteristic function estimates, and in this wise the consistency of the estimators used in this section.
} 
In place of the cumulants the characteristic function is also available to demonstrate one-dimensional dilative stability and to estimate the LRD parameter $H$. Taking the logarithm twice and then the real part in (3.44), we have

$$
\operatorname{Re} \log \log \varphi_{\frac{J(t)}{t}}(u)=2(H-1) \log t+\operatorname{Re} \log \log \varphi_{J(1)}(u) .
$$

Changing to the notation of Corollary 4, this means that

$$
\operatorname{Re} \log \log \varphi_{\Delta J^{\left(n_{i}\right)}}(u)=2(H-1) \log n_{i}+\operatorname{Re} \log \log \varphi_{\Delta J^{(1)}}(u) .
$$

When testing the ATM data from this point of view, we used the same range of the levels of aggregation $n_{i}$ as above. The arguments $u$ of the estimated characteristic functions were 100 points equally spaced in the interval $(0,0.05]$. (The reason for the choice of 0.05 as the end point is that the variance of the estimated characteristic function and also the variance of the curves increase apace for larger values of $u$.) Figure 2 plots for each fixed level of aggregation $n_{i}$ the left-hand side of (4.3) as a function of $u$. If the ATM data set were a realization of the $\triangle$ LISDLG process, then by (4.3) these curves should be approximately parallel. And it seems they really are.

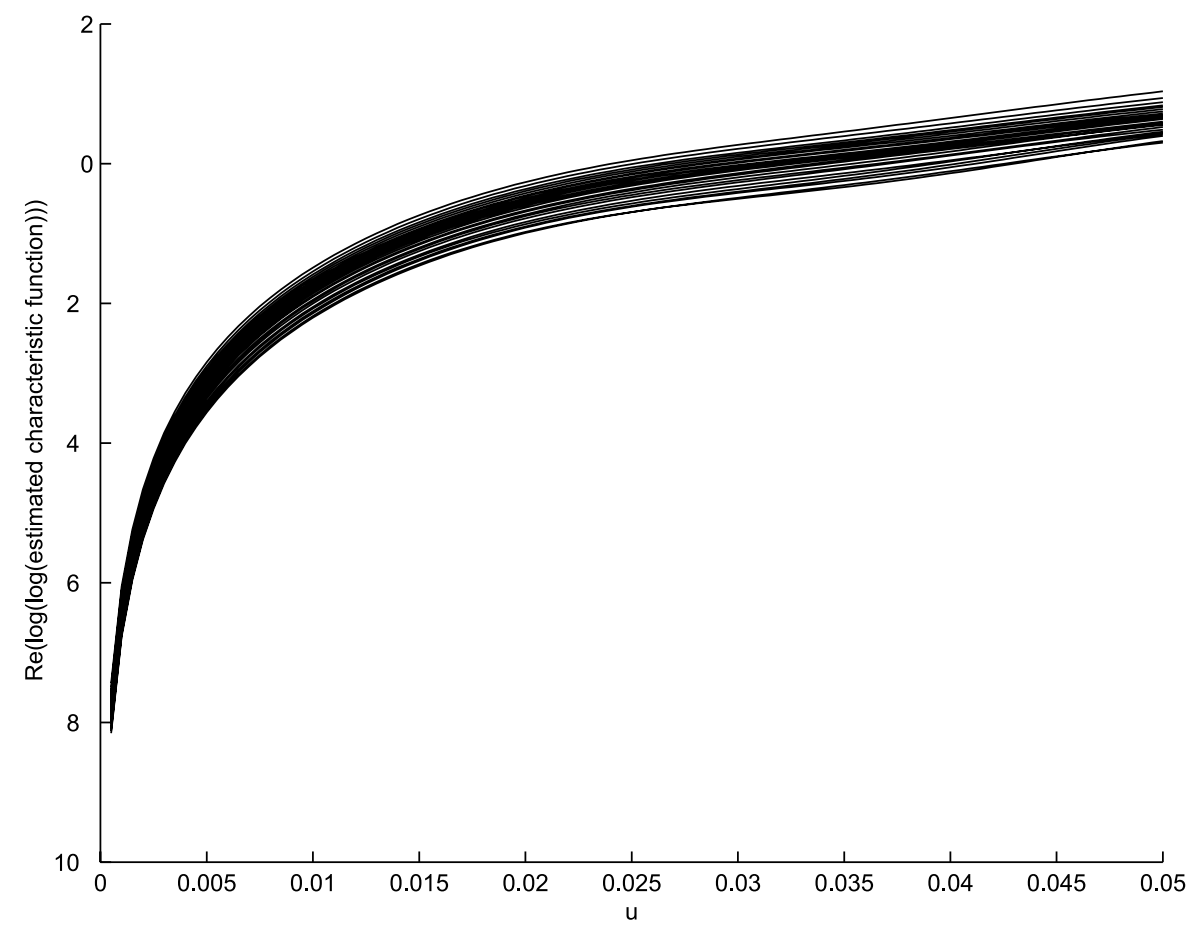

Figure 2. One-dimensional dilative stability. Each curve is the graph of a function Re $\log \log \widehat{\varphi}_{\Delta J^{\left(n_{i}\right)}}(u)$ as function of $u$. The aggregational levels $n_{i}$ are the same as in Figure 1. The curves are approximately parallel.

Figure 3 plots for different values of $u$ the left-hand side of (4.3) as a function of $\log n_{i}$. If the ATM data set were a realization of the $\triangle$ LISDLG process, then by (4.3) these curves should be approximately parallel lines, each with the approximate slope $2(H-1)$. And it seems they really are.

If the data come from a $\triangle$ LISDLG process, the previous method can be used to estimate parameter $H$ from the linear model (4.3). We also tried it for wider ranges of the arguments of characteristic function. Figure 4 shows the result when 500 equidistant values of $u_{j}$ in the interval $(0,10)$ are used. The mean of the 500 estimated values $\widehat{H}\left(u_{j}\right), \mathrm{j}=1, \ldots, 500$, is

$$
\widehat{H}=0.9452 \text {. }
$$




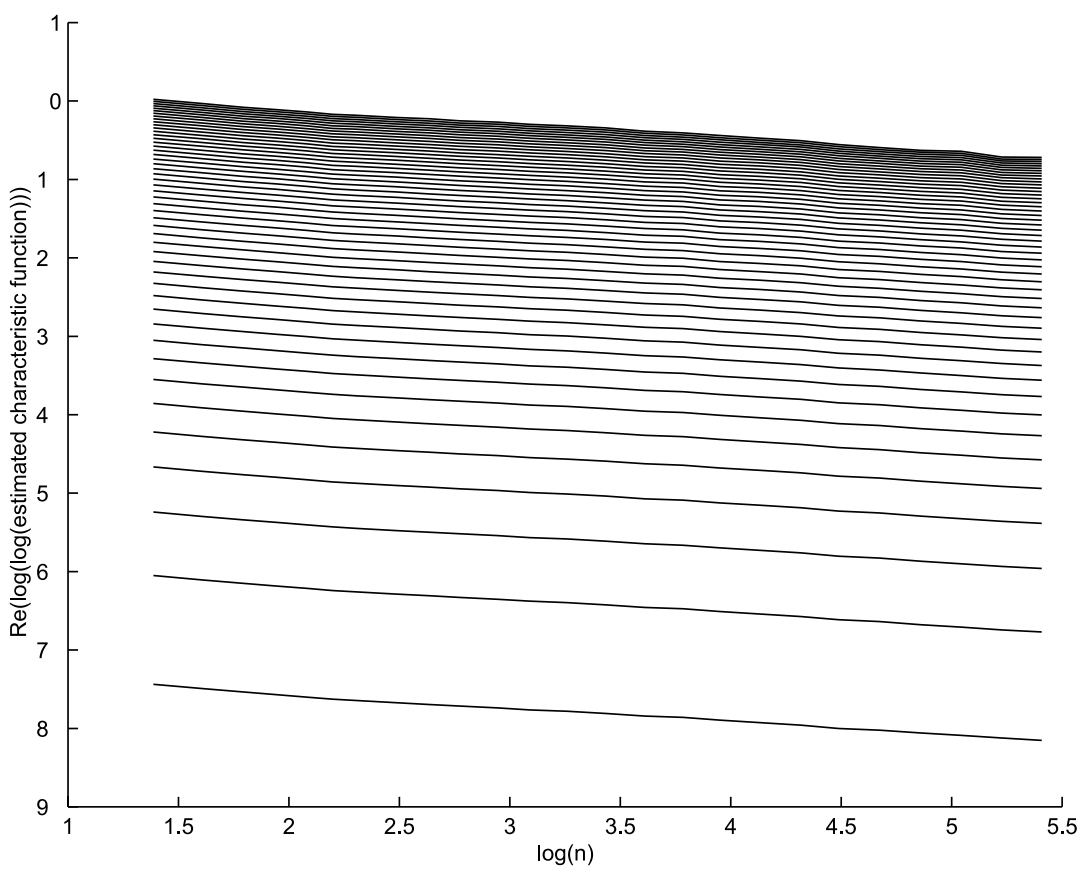

Figure 3. One-dimensional dilative stability. Each line is the graph of a function Re $\log \log \widehat{\varphi}_{\Delta J^{\left(n_{i}\right)}}(u)$ as function of $\log n_{i}$. The aggregational levels $n_{i}$ are the same as in Figures 1 and 2. The values of $u$ are the same as in Figure 2. Each line has approximately the same slope $2(H-1)$.

If even larger intervals are used in place of $(0,10)$, the arrangement of the estimations $\widehat{H}\left(u_{j}\right)$ remains similar to the one in Figure 4. Thus, we have good reason for supposing the value in (4.4) to be an adequate estimation of parameter $H$.

\subsection{Fitting of distributions and spectra}

\subsubsection{Fitting of the one-dimensional distributions}

Figure 5 shows the histogram of the ATM data centralized to zero and the density function of the shifted $\Gamma$ distribution fitted by the method of moments using the second and third moments. Though it is known that the distribution of the $\Delta$ LISDLG process $\Delta J(t)$ is not exactly shifted $\Gamma$, it is close to it if the LRD parameter $H$ is near to 1 , see Remark 14 . We do not know how rapid the convergence is; anyhow it seems that the estimated value $\widehat{H}=0.9452$ in (4.4) is sufficiently close to 1 , the distribution of $\Delta J(t)$ being near to the shifted $\Gamma$.

Let us now examine what the fitting looks like for characteristic functions. We know the characteristic function of $\Delta J(1)$ only in the interval $\left(-\sigma_{0}^{-2}, \sigma_{0}^{-2}\right)$, where the series expansion of its logarithm holds, see Proposition 15. Using the value (4.4) for $H$, we searched for the values of the other two parameters $c_{0}$ and $\sigma_{0}^{2}$ giving the best least squares fitting, in the interval $\left(-0.9 \sigma_{0}^{-2}, 0.9 \sigma_{0}^{-2}\right)$, between the characteristic function $\varphi_{\Delta J(1)}(u)$ and the empirical one $\widehat{\varphi}_{\Delta J(1)}(u)$, the latter being estimated from the ATM data. (The coefficient 0.9 is used because the series expansion begins to diverge, or rather, to converge slowly near the end points of the convergence interval. This effect occurs even for the series expansion of the log-characteristic function of the $\Gamma$ distribution.) The search ended on finding the optimal values

$$
\begin{aligned}
& \widehat{c_{0}}=12.7201 \\
& \widehat{\sigma_{0}^{2}}=62.1118,
\end{aligned}
$$




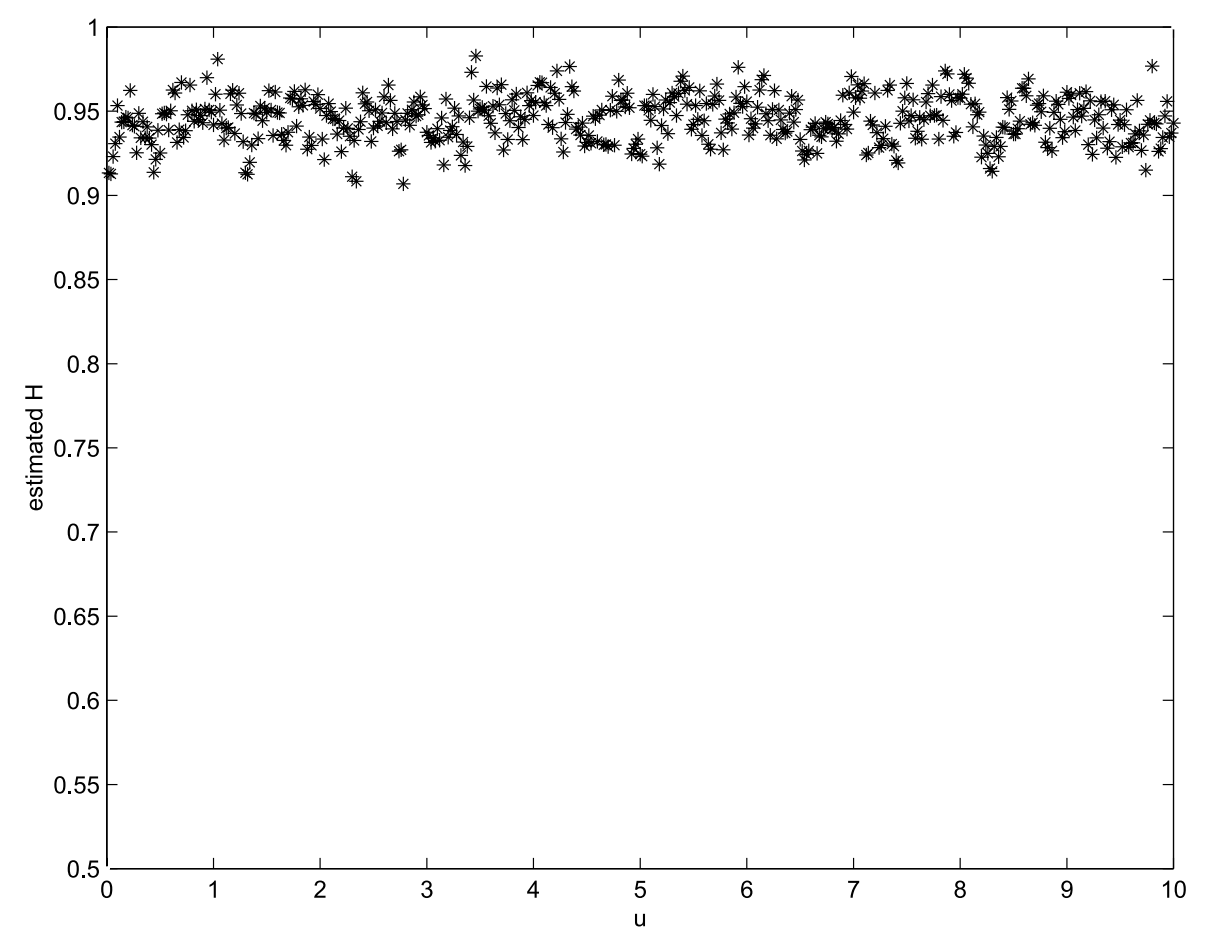

Figure 4. Linear regression estimators of $H$ based on the linear model (4.3). The points have coordinates $\left(u_{j}, \widehat{H}\left(u_{j}\right)\right)$, where $u_{j}, \mathrm{j}=1, \ldots, 500$, are equidistant in the interval $(0,10)$, and $\widehat{H}\left(u_{j}\right)$ is the linear regression estimator based on (4.3) when the range of the aggregational levels $n_{i}$ is the same as in Figure 1.

and the best fitting can be seen in Figure 6. The value giving the end points of the interval used at the final stage is $0.9{\widehat{\sigma_{0}^{2}}}^{-1}=0.0145$. The curve consisting of discrete points is the estimated characteristic function $\widehat{\varphi}_{\Delta J(1)}(u)$, and the best fitting $\varphi_{\Delta J(1)}(u)$ is the continuous curve. Also the best fitting $\Gamma$ characteristic function is plotted, but it is not shown as it is overlapped by the other optimal curve.

\subsubsection{Fitting of the spectra and bispectra}

To demonstrate the fitting of the theoretical spectra of the $\triangle$ LISDLG process to the empirical spectra, we use the $\approx 1 \mathrm{~s}$ level ATM data because the coincidence of the second order spectra is rather convincing at this level of aggregation. Thus, the level of aggregation of the $\Delta$ LISDLG process too has to be changed by a factor of 10. Or rather, we can get the same result changing not the level of aggregation, but parameters $c_{0}$ and $\sigma_{0}^{2}$, according to the scaling behavior (3.33) or (3.34) of the cumulants. For this purpose, consider the parameters of the $\triangle$ LISDLG process fitted above to the $\approx 0.1 \mathrm{~s}$ level ATM data, i.e.

$$
\begin{aligned}
H & =0.9452 \\
c_{0} & =12.7201 \\
\sigma_{0}^{2} & =62.1118,
\end{aligned}
$$

see (4.4) and (4.5). Now the parameters of the $\triangle$ LISDLG process, after changing the time scale by a factor of 10 , are

$$
\begin{aligned}
H & =0.9452 \\
c_{0} & =12.7201 \cdot 10^{2(H-1)}=9.883 \\
\sigma_{0}^{2} & =62.1118 .
\end{aligned}
$$




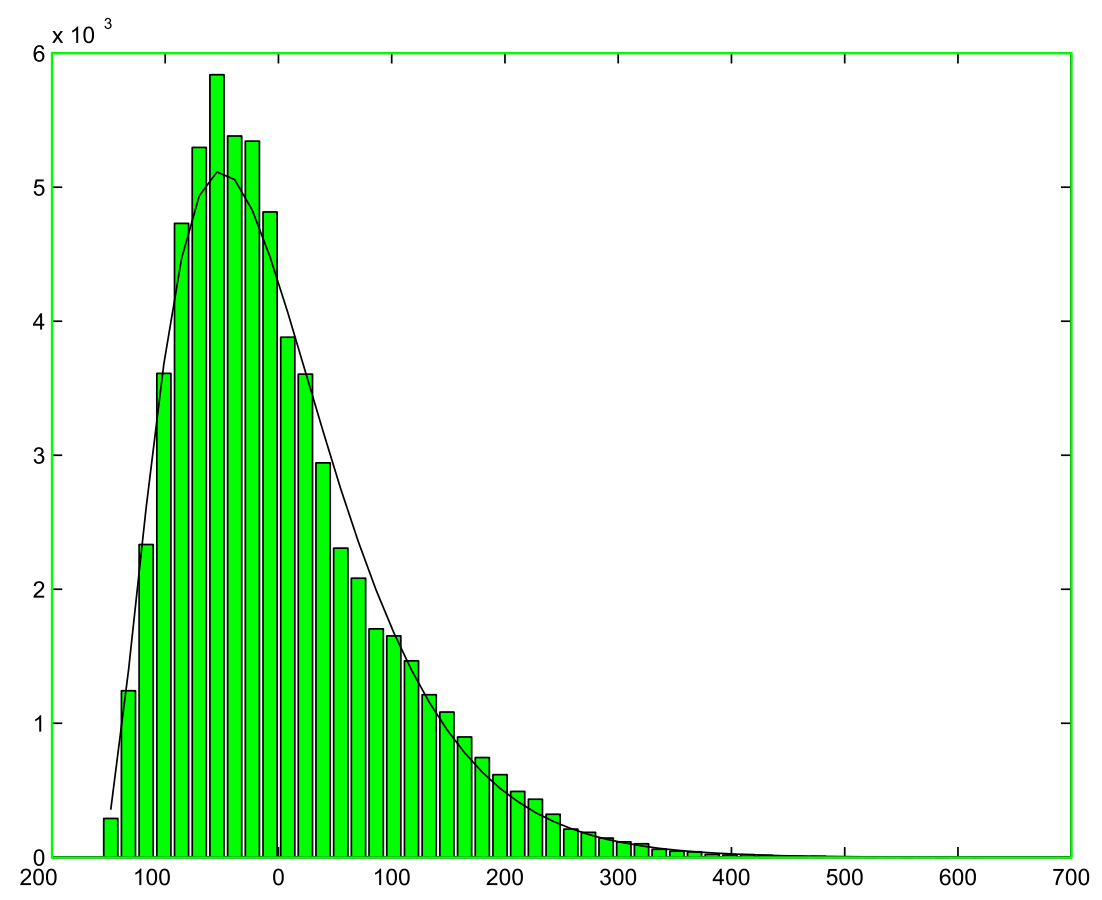

Figure 5. Histogram of the ATM data centralized to zero and the shifted $\Gamma$ density fitted to them.

Thus when computing the theoretical spectrum and bispectrum by the corresponding formulas (3.76) and (3.91), respectively, we used the parameters in (4.6).

Figure 7 shows the fitting of the estimated spectrum alias smoothed periodogram and the theoretical spectrum (3.76) of the $\triangle$ LISDLG process. More precisely, in Figure 7 both spectra are normed so that their geometric mean be equal to 1 .

Figure 8 shows is the theoretical bispectrum. Figures 9 and 10 show the real part and the imaginary part, respectively, of the estimated bispectrum. More precisely, in Figures 8, 9 and 10 both the theoretical and the empirical bispectrum are normed so that the arithmetic mean of their real parts be equal to 1 . Now observe the imaginary part of the estimated bispectrum (Fig. 10) fluctuating around zero with a small amplitude as compared to the range of the real part (Fig. 9). Thus, the imaginary part seems to be negligible. This fits in with the fact that the imaginary part of the theoretical bispectrum is zero. Also note the similarity of the real parts, see Figures 8 and 9.

\section{Open PROBLEMS AND DIRECTIONS OF FUTURE RESEARCH}

It seems to be a difficult task to calculate

$$
\int_{[0,1]^{m}} D_{\tau_{0}}(\underline{s})^{2(H-1)} \mathrm{d} \underline{s}
$$

in closed form, in order to get the form of the dependence on dimension $m$. The difficulty arises from the fact that for $m \geqslant 4$ the term $D_{\tau_{0}}(\underline{s})$ is not symmetric in its variables, hence for different permutations $\tau$ the integrals on the simplexes $\left\{\underline{s} \in[0,1]^{m}: s_{\tau_{1}} \leqslant \ldots \leqslant s_{\tau_{m}}\right\}$ are different as a rule. This is a relevant problem because the $m$-th cumulant of the distribution of the LISDLG process $J(t)$ differs in the factor (5.1) from the $m$-th cumulant of the corresponding $\Gamma$ distribution, see (3.33).

A problem connected with the previous one is to specify the distribution of the LISDLG process $J(t)$, and hence that of the $\Delta$ LISDLG process $\Delta J(t)$ (for fixed $t$, naturally). Because of the one-dimensional dilative stability, see Proposition 17, it would suffice to give the distribution of $J(1)$. The authors know the 

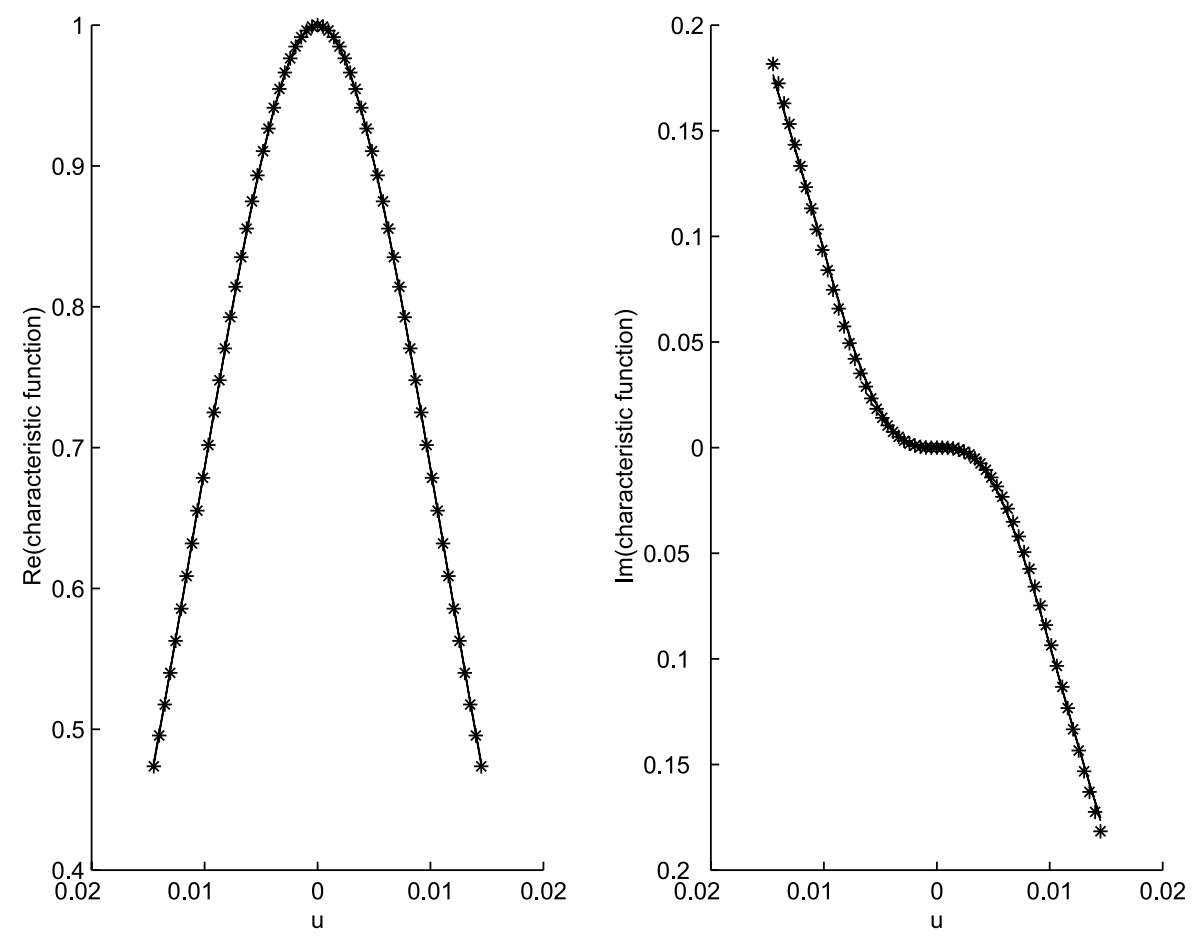

FiguRE 6. Real (left) and imaginary (right) parts of the following three characteristic functions: the empirical one estimated from ATM data (discrete points); the best fitting $\triangle$ LISDLG and the best fitting $\Gamma$ characteristic fuction (both plotted with continuous lines and exactly overlapped by each other). When fitting the $\triangle$ LISDLG characteristic function, parameter $H$ was fixed at the value given by (4.4). The parameters yielding the best fitting characteristic function $\varphi_{\Delta J(1)}(u)$ are the ones in (4.5). The end points of the interval used for the optimizing procedure are $\pm 0.9{\widehat{\sigma_{0}^{2}}}^{-1}$, which are \pm 0.0145 at the final stage.

series expansion of the log characteristic function only in a certain neighborhood of zero, see Proposition 15. Reference [10] gives an elegant closed-form expression for the whole characteristic function of the integral on $[0, t]$, of a squared OU process. Using it as well as the mixture representation of the logarithm of the characteristic function, one obtains

$$
\log \varphi_{J_{\lambda}(1)}(u)=\frac{\mu_{0} \lambda^{3-2 H}}{\Gamma(3-2 H) \sigma_{0}^{2}} \int_{0}^{\infty} x^{2(1-H)} \mathrm{e}^{-\lambda x}\left(\frac{-i u \sigma_{0}^{2}}{x}+g(u, x)\right) \mathrm{d} x
$$

where

$$
g(u, x) \stackrel{\circ}{=}-\frac{1}{x} \log \left[\frac{1}{2}\left(z(u, x)+\frac{1}{z(u, x)}\right) \sinh \left(\frac{x}{2} z(u, x)\right)+\cosh \left(\frac{x}{2} z(u, x)\right)\right]
$$

and

$$
z(u, x)=\sqrt{1-\frac{4 i u \sigma_{0}^{2}}{x}} .
$$

Taking limit in (5.2) the characteristic function $\varphi_{J(1)}(u), u \in \mathbb{R}$, could be derived, since

$$
\log \varphi_{J(1)}(u)=\lim _{\lambda \rightarrow 0} \log \varphi_{J_{\lambda}(1)}(u),
$$




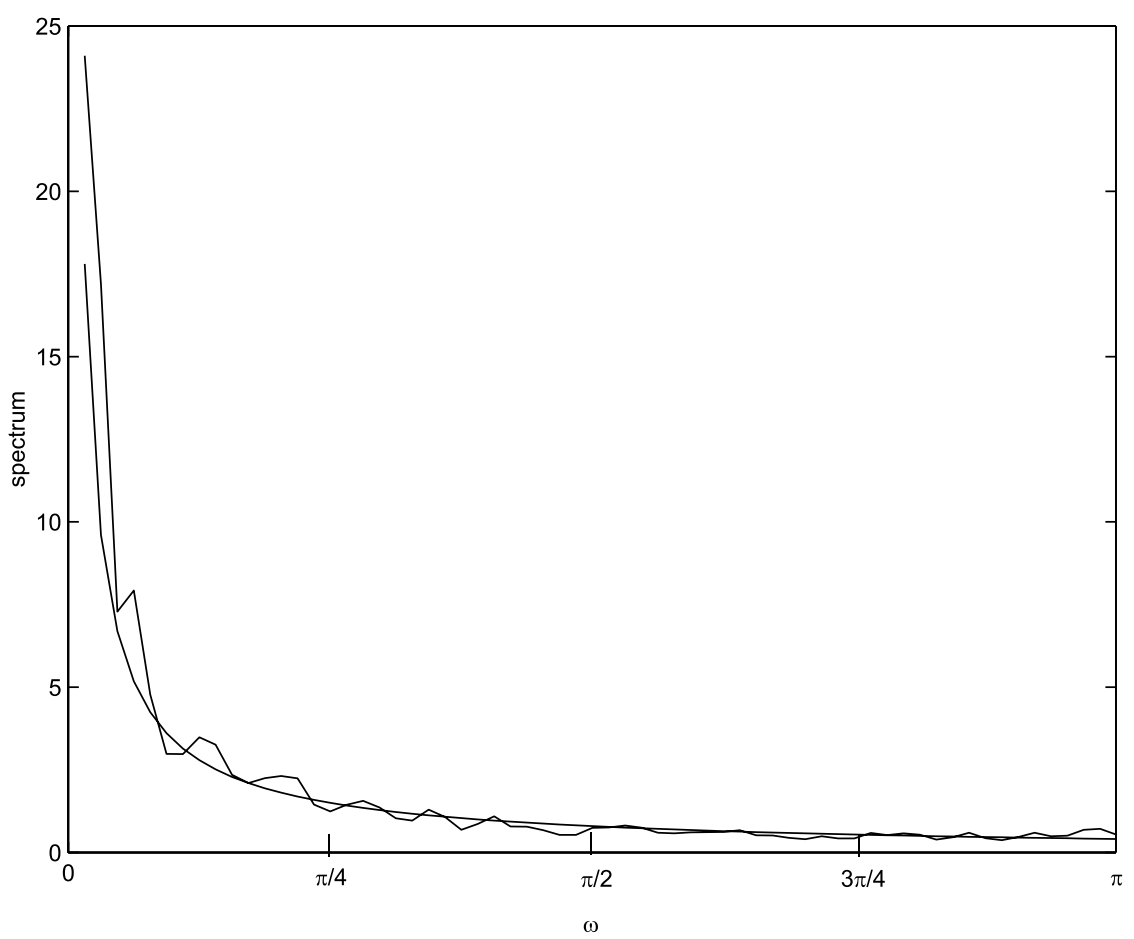

FIGURE 7 . The smoothed periodogram of the $\approx 1 \mathrm{~s}$ level ATM data and the theoretical spectrum (3.76) of the $\triangle$ LISDLG process with parameters as in (4.6). Both curves are normed so that their geometric mean be 1 .

$u \in \mathbb{R}$, if the function

$$
x \longmapsto x^{2(1-H)}\left(\frac{-i u \sigma_{0}^{2}}{x}+g(u, x)\right),
$$

$x \in \mathbb{R}_{+}$, proved to be integrable ${ }^{5}$.

Note also that expression (5.1) in closed form would be useful for deriving the Fourier transform of the Lévy measure in the Lévy-Khinchin representation of $\log \varphi_{J(1)}{ }^{6}$.

Concerning the spectra of order $m \geqslant 4$, it follows easily from Definition 9 and Remark 17 that they must be real provided they exist, similarly to the case $m=3$, i.e. the case of the bispectrum. The question is whether they exist, and if they do, is there a general form of the $m$-th order spectrum as a function of $m$ too? This problem is at least as hard as to compute (5.1) in closed form, because it needs the knowledge of the $m$-th order spectrum of the DLG process. The latter however makes it necessary to calculate an integral, similar to but even more complicated than, equation (5.1), see the proof of Proposition 9. Anyway, the authors know the closed expression (3.37) for the cumulants of the derivative of the LISDLG process $J(t)$. Though the derivative of the LISDLG process $J(t)$ is only an informal thing, its cumulants can be defined exactly. Is there a closed form expression, similar to $(3.37)$, for cum $\left(\Delta J(0), \Delta J\left(t_{1}\right), \ldots, \Delta J\left(t_{m-1}\right)\right)$ which also holds for all $m \geqslant 4$ ?

Another direction for future research is the fitting of $\triangle$ LISDLG processes to simulated and real data. First of all, concerning the simulation it must be noted that there is a very effective method to simulate a DLG process in discrete time points, see Remark 4. Probably, one can put this method to use for simulating

\footnotetext{
${ }^{5}$ After submitting the first version of this paper, the authors pushed through this idea, so the cumulant function log $\varphi_{J}(1)(u)$, $u \in \mathbb{R}$, has been obtained since then.

${ }^{6}$ The referee was so kind to draw the authors attention to this fact.
} 


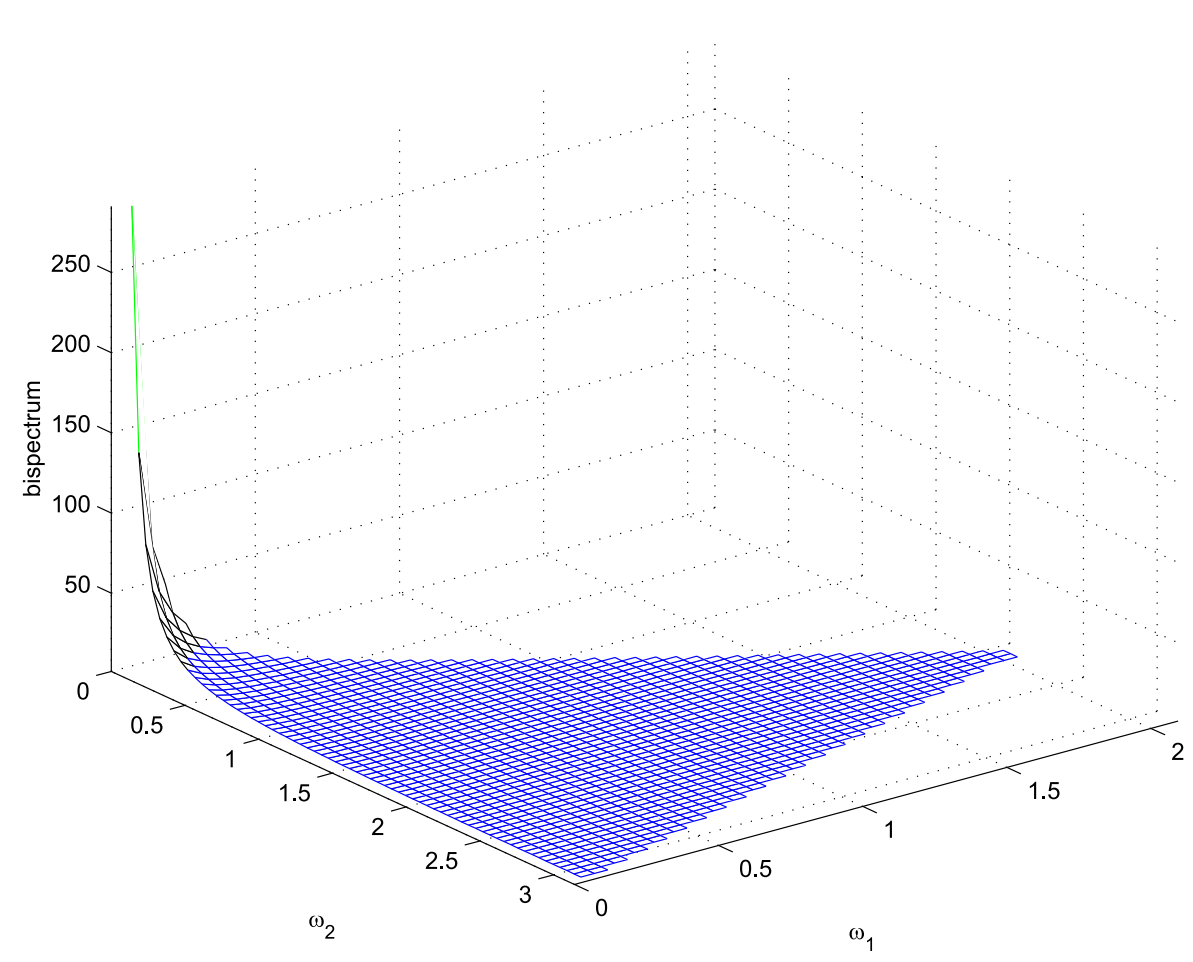

FiguRE 8 . The theoretical bispectrum (3.91) of the $\triangle$ LISDLG process with parameters as in (4.6). It is normed so that its arithmetic mean be 1 . It is plotted on the determining triangle with vertices $(0,0),(\pi, 0),(2 \pi / 3,2 \pi / 3)$, see [37] (p. 29).

$\Delta$ LISDLG process $^{7}$. Secondly, adapting existing parameter estimation methods and tests of model fitting to our process is a promising task, as well as contriving and investigating new ones, including those we used in Section 4.

Stochastic modelling of computer network data traffic flow processes using the $\Delta$ LISDLG process is another topic for future research. The motivation comes not only from the apparently convincing fitting found by using various graphical methods and several data sets, but also from the existing realistic models for the DLG processes which are the component processes for our superposition step. In particular, the DLG process is a CBI process with a.s. continuous trajectories, see Theorem 1, and the latter process can be represented as a scaled limit of Galton-Watson processes with immigration, see Theorem 2.2 in [20]. Another practical model is a Cox process with DLG process intensity, resulting in a birth and death process with immigration, see [7].

\section{Appendix A. Multivariate $\Gamma$ Distribution}

\section{The accompanying Gaussian distribution}

Let $m \in \mathbb{N}$, and let $\Sigma=\left(\sigma_{i j}\right)_{i, j=1, \ldots, m}$ be an $m \times m$ matrix with the following property:

$$
\Sigma \text { is symmetric, positive definite and } \sigma_{11}=\ldots=\sigma_{m m}=\sigma^{2} \text {. }
$$

Let $\mathbf{X}=\left(X_{1}, X_{2}, \ldots, X_{m}\right)$ be a zero mean Gaussian vector with covariance matrix $\Sigma$. Define $\mathbf{Y}=\left(Y_{1}, Y_{2}\right.$, $\left.\ldots, Y_{m}\right)$ as the random vector with entries $Y_{k}=X_{k}^{2}, k=1,2, \ldots, m$. The characteristic function of $\mathbf{Y}$ is

$$
\varphi_{\mathbf{Y}}(\underline{u})=|I-2 i U \Sigma|^{-1 / 2}
$$

\footnotetext{
${ }^{7}$ After submitting the first version of this paper, the authors pushed through this idea. More specifically, processes approximating the $\triangle$ LISDLG process with adequate precision have been simulated.
} 


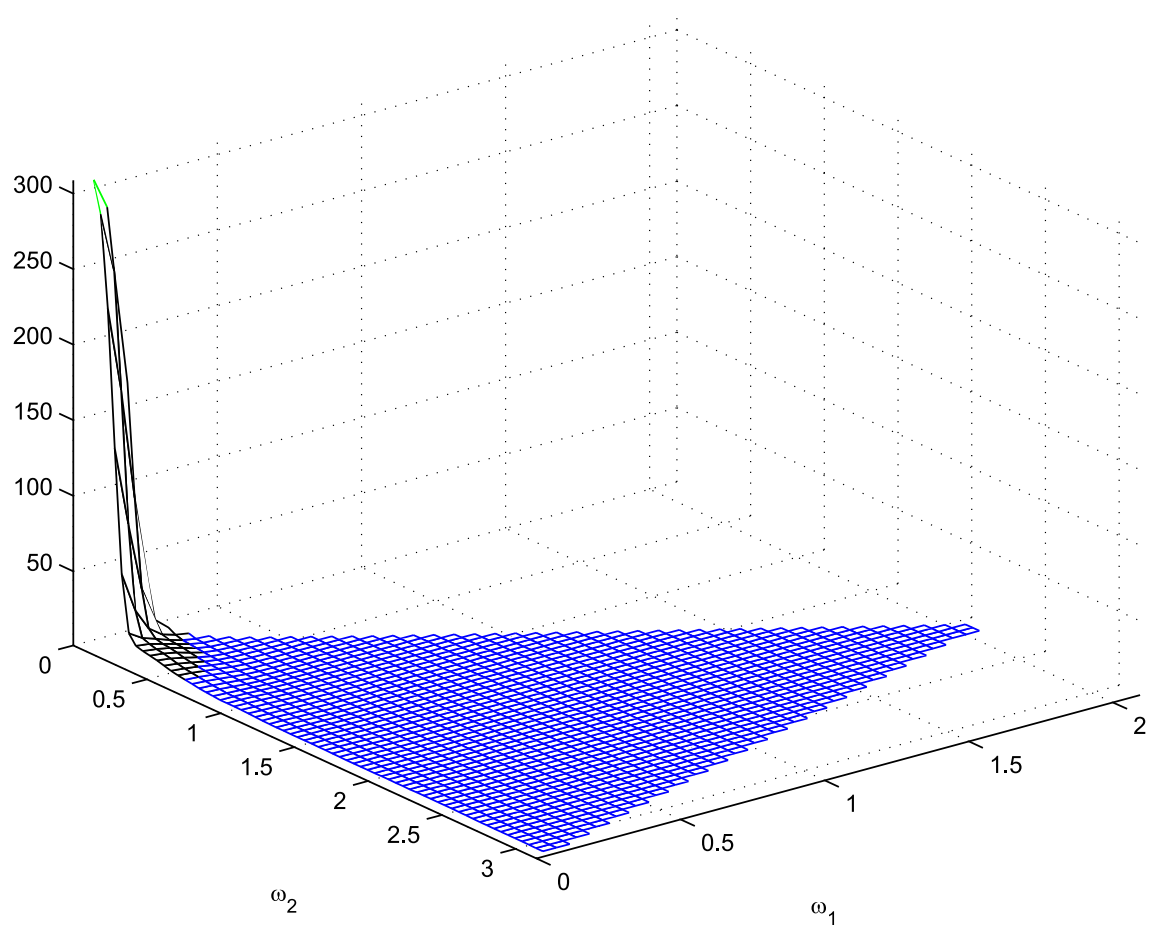

FiguRE 9. The real part of the estimated bispectrum of the $\approx 1 \mathrm{~s}$ level ATM data. It is normed so that its arithmetic mean be equal to 1 . It is plotted on the same triangle as in Figure 8 .

where $U \stackrel{\circ}{=} \operatorname{diag}(\underline{u})$ with $\underline{u} \in \mathbb{R}^{m}$, i.e. a matrix with zero elements except for the main diagonal, which is $\underline{u}$, $I$ is the $m \times m$ identity matrix, and || denotes the determinant. If one takes $\mathbf{Z}=\sum_{k=1}^{n} \mathbf{Y}_{k}$, the sum of $n \in \mathbb{N}$ independent copies of the vector $\mathbf{Y}$, then one gets the characteristic function

$$
\varphi_{\mathbf{Z}}(\underline{u})=|I-2 i U \Sigma|^{-n / 2} \text {. }
$$

All the marginals of $\varphi_{\mathbf{Z}}(\underline{u})$ are $\chi^{2}$ distributed with degree of freedom $n$ which is a particular case of the $\Gamma$ distribution. The $\Gamma(\alpha, \beta)$ distribution is defined in general either by the density

$$
f_{\Gamma(\alpha, \beta)}(x)=\frac{x^{\alpha-1}}{\beta^{\alpha} \Gamma(\alpha)} \exp (-x / \beta), \text { if } x>0 \text { and zero otherwise }
$$

or by the characteristic function

$$
\varphi_{\Gamma(\alpha, \beta)}(u)=(1-i u \beta)^{-\alpha}
$$

Its cumulants are

$$
\operatorname{cum}_{m}(\Gamma(\alpha, \beta))=(m-1) ! \alpha \beta^{m}
$$

$m=1,2,3, \ldots$ Parameters $\alpha>0$ and $\beta>0$ are called the shape parameter and the scale parameter, respectively. In that context the marginals of $\varphi_{\mathbf{Z}}(\underline{u})$ are $\Gamma\left(n / 2,2 \sigma^{2}\right)$. The entries of $\mathbf{Y}$ are correlated and $\chi^{2}$ distributed with degree of freedom 1. The cumulants of $\mathbf{Y}$ are the same as the cumulants of $\mathbf{Y}-\sigma^{2}=$ $\left(H_{2}\left(X_{1}\right), H_{2}\left(X_{2}\right), \ldots, H_{2}\left(X_{m}\right)\right)$, where $H_{2}\left(X_{k}\right)=X_{k}^{2}-\sigma^{2}$ is the Hermite polynomial of second order. The cumulants of Hermite polynomials are well-known, in particular in the following case. 


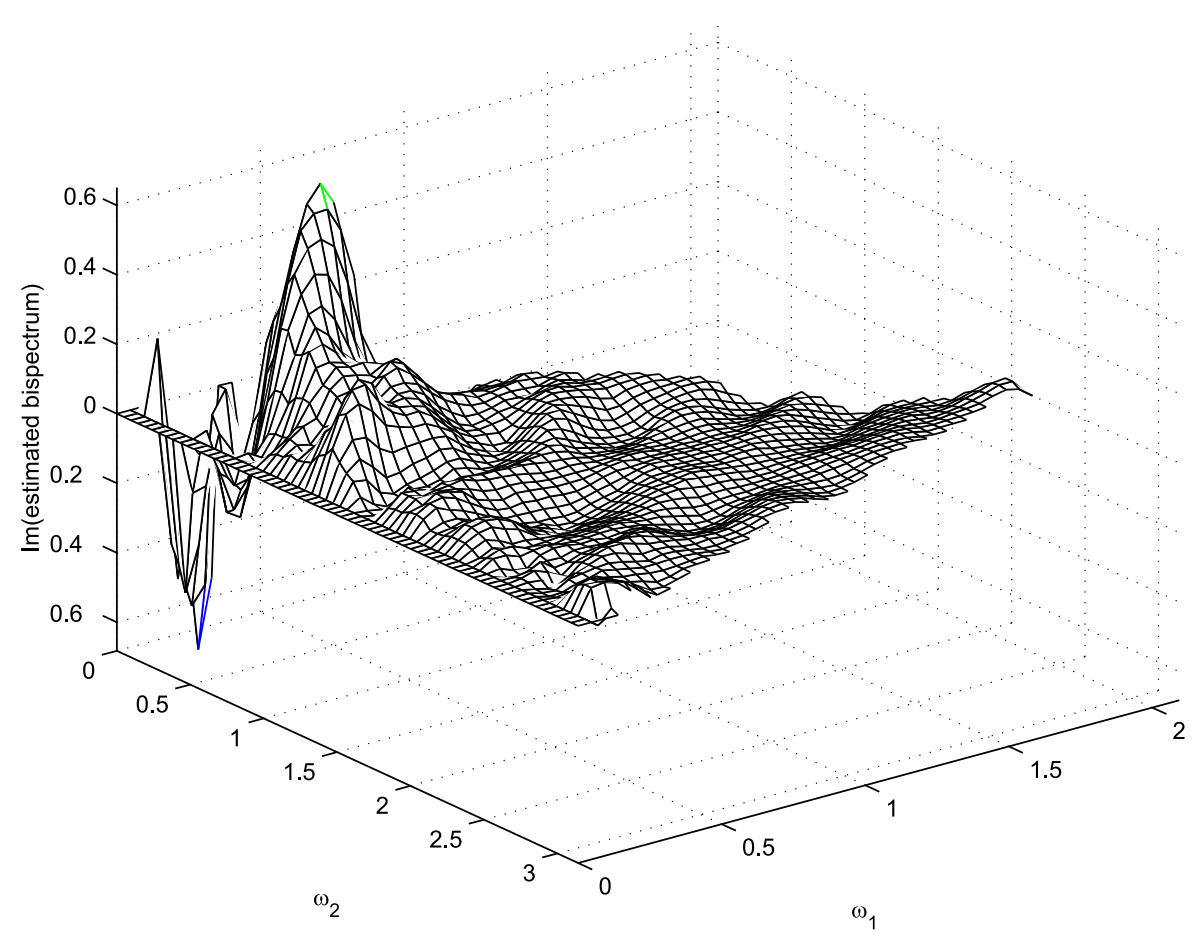

Figure 10. The imaginary part of the estimated bispectrum of the $\approx 1 \mathrm{~s}$ level ATM data. It is normed by the same constant with which the real part. It is plotted on the same triangle as in Figure 8.

\section{Proposition 27.}

$$
\operatorname{cum}\left(H_{2}\left(X_{1}\right), H_{2}\left(X_{2}\right), \ldots, H_{2}\left(X_{m}\right)\right)=
$$

see [40], Example 10, for details.

E.g., if $m=2$, one can see that

$$
\operatorname{cum}\left(H_{2}\left(X_{1}\right), H_{2}\left(X_{2}\right)\right)=\operatorname{cov}\left(H_{2}\left(X_{1}\right), H_{2}\left(X_{2}\right)\right)=2 \sigma_{12}^{2},
$$

and if $m=3$,

$$
\operatorname{cum}\left(H_{2}\left(X_{1}\right), H_{2}\left(X_{2}\right), H_{2}\left(X_{3}\right)\right)=8 \sigma_{12} \sigma_{23} \sigma_{31} .
$$

Although one cannot change the exponent $n / 2$ in (A.2) for an arbitrary positive real number $\alpha$, because the result might not be a characteristic function, see [24], we define the function

$$
\varphi(\underline{u})=|I-2 i U \Sigma|^{-\alpha},
$$

for further consideration.

Definition 11. If function $\varphi(\underline{u})$ defined by (A.3) is a characteristic function, then the $m$-dimensional $\Gamma$ distribution with shape parameter $\alpha$ and scale parameter matrix $2 \Sigma$ according to the accompanying Gaussian distribution, $\Gamma(\alpha, 2 \Sigma)$ for short, is defined, in particular by its characteristic function $\varphi(\underline{u})$. 
Let $\mathbf{Z}=\left(Z_{1}, \ldots, Z_{m}\right)$ be $\Gamma(\alpha, 2 \Sigma)$ distributed. It is easy to see that each component of $\mathbf{Z}$ has onedimensional $\Gamma$ distribution, namely,

$$
Z_{k} \sim \Gamma\left(\alpha, 2 \sigma^{2}\right)
$$

$k=1, \ldots, m$. Moreover, for $1 \leqslant k \leqslant m$, each subset $\left(Z_{i_{1}}, \ldots, Z_{i_{k}}\right)$ also has $k$-dimensional $\Gamma$ distribution. Naturally, there are other $m$-dimensional distributions whose one-dimensional marginals are $\Gamma$ distributions, however the one given above is the most plausible of them, because of the following:

Proposition 28 (see Th. 1 in [24]). The distribution $\Gamma(n / 2,2 \Sigma)$ is just the marginal distribution of the diagonal elements of a Wishart matrix.

For these special values of the shape parameter, i.e. for half-integer positive $\alpha$, and arbitrary $\Sigma$ with property (A.1), the multi-dimensional $\Gamma(\alpha, 2 \Sigma)$ distribution was defined in [25]. Reference [24] draws our attention to the fact that for $m \geqslant 2$, if one makes no restraints for the parameters, other than $\alpha>0$ and $\Sigma$ fulfils (A.1), then (A.3) is not necessarily a characteristic function. However, the infinite divisibility of the $\Gamma(1 / 2,2 \Sigma)$ distribution is a necessary and sufficient condition for the $\Gamma(\alpha, 2 \Sigma)$ distribution to exist for all $\alpha>0$. Reference [14] gave a sufficient condition for infinite divisibility, namely that $\Sigma$ is the autocovariance matrix of a Gauss-Markov vector. The latter condition comes true for the very case of an autocovariance matrix of a stationary OU process. To conclude, we state the following:

Proposition 29 (see [14]). If $\alpha>0$ and $\Sigma$ is the autocovariance matrix of the vector $\left(X\left(t_{1}\right), \ldots, X\left(t_{m}\right)\right)$, where $t_{1}<\ldots<t_{m}$ and $X(t)$ is a stationary OU process, then the $\Gamma(\alpha, 2 \Sigma)$ distribution really exists.

The cumulants of distribution $\Gamma(\alpha, 2 \Sigma)$ are the same as the cumulants of $\Gamma(n / 2,2 \Sigma)$ but for some multiplicative constant because the cumulant generating function is $\log \varphi(\underline{u})$. Let $\mathbf{Z}=\left(Z_{1}, \ldots, Z_{m}\right)$ be $\Gamma(\alpha, 2 \Sigma)$ distributed, then

$$
\begin{aligned}
\operatorname{cum}\left(Z_{1}, Z_{2}, \ldots, Z_{m}\right) & =2 \alpha \operatorname{cum}\left(H_{2}\left(X_{1}\right), H_{2}\left(X_{2}\right), \ldots, H_{2}\left(X_{m}\right)\right) \\
& =2^{m} \alpha \sum_{\left(i_{1}, \ldots, i_{m-1}\right) \in \operatorname{Perm}(2, \ldots, m)} \sigma_{1 i_{1}} \sigma_{i_{1} i_{2}} \ldots \sigma_{i_{m-1} 1},
\end{aligned}
$$

where $\mathbf{X}=\left(X_{1}, X_{2}, \ldots, X_{m}\right)$ is the accompanying Gaussian random vector. The cumulants (A.4) are invariant under changing the signs of the $X$ 's, therefore the distribution $\Gamma(\alpha, 2 \Sigma)$ does not define the accompanying Gaussian distribution uniquely.

The authors are grateful to the referee for the careful review and the constructive attitude.

\section{REFERENCES}

[1] O.E. Barndorff-Nielsen, Superposition of Ornstein-Uhlenbeck type processes, Technical Report 1999-2, MaPhySto. Aarhus University (1999), URL: http://www.maphysto.dk/cgi-bin/w3-msql/publications/.

[2] J. Beran, Statistics for Long-Memory Processes. Chapman \& Hall, Monogr. Statist. Appl. Probab. 61 (1994).

[3] D.R. Brillinger, An introduction to polyspectra. Ann. Math. Statist. 36 (1965) 1351-1374.

[4] L. Calvet and A. Fisher, Forecasting multifractal volatility. J. Econometrics 105 (2001) 27-58.

[5] P. Carmona and L. Coutin, Fractional Brownian motion and the Markov property, Electron. Commun. Probab. 3 (1998) 95-107.

[6] P. Carmona, L. Coutin and G. Montseny, Applications of a representation of long-memory Gaussian processes. Stochastic Process. Appl. (submitted), URL: http://www.sv.cict.fr/lsp/Carmona/prepublications.html

[7] P. Clifford and G. Wei, The equivalence of the Cox process with squared radial Ornstein-Uhlenbeck intensity and the death process in a simple population model. Ann. Appl. Probab. 3 (1993) 863-873.

[8] D.R. Cox, Long-range dependence, non-linearity and time irreversibility. J. Time Ser. Anal. 12 (1991) 329-335.

$[9]$ J.C. Cox, J.E. Ingersoll and S.A. Ross, A theory of the term structure of interest rates. Econometrica 53 (1985) 385-407.

[10] T. Dankel Jr., On the distribution of the integrated square of the Ornstein-Uhlenbeck process. SIAM J. Appl. Math. 51 (1991) 568-574.

[11] E.B. Dynkin, Markov Processes, Vols. 1-2. Springer-Verlag, Berlin-Göttingen-Heidelberg (1965).

[12] W. Feller, Two singular diffusion problems. Ann. Math. (2) 54 (1951) 173-182.

[13] C. Granger, Long-memory relationship and aggregation of dynamic models. J. Econometrics 14 (1980) 227-238.

[14] R.C. Griffiths, Infinitely divisible multivariate gamma distributions. Sankhya Ser. A 32 (1970) 393-404. 
[15] E. Iglói, Long-range dependent processes with real bispectrum are third order nonlinear, Technical Report 259 (2001/1). University of Debrecen, Institute of Mathematics and Informatics (2001).

[16] E. Iglói and G. Terdik, Long-range dependence through Gamma-mixed Ornstein-Uhlenbeck process. Electron. J. Probab. (EJP) 4 (1999) 1-33.

[17] N. Ikeda and S. Watanabe, Stochastic differential equations and diffusion processes. North-Holland Publishing Co., Amsterdam (1981).

[18] S. Jaffard, The multifractal nature of Lévy processes. Probab. Theory Related Fields 114 (1999) 207-227.

[19] S. Karlin and J. McGregor, Classical diffusion processes and total positivity. J. Math. Anal. Appl. 1 (1960) 163-183.

[20] K. Kawazu and S. Watanabe, Branching processes with immigration and related limit theorems. Teor. Verojatnost. Primenen. 16 (1971) 34-51.

[21] A.N. Kolmogorov, Über die analitischen Methoden in der Wahrscheinlichkeitsrechnung. Math. Ann. 104 (1931) 415-458.

[22] A.N. Kolmogorov, Wiener spiral and some other interesting curve in Hilbert space. C. R. Acad. Sci. URSS 26 (1940) $115-118$.

[23] A.N. Kolmogorov, The local structure of turbulence in incompressible viscous fluid for very large Reynolds numbers. $C$. R. Acad. Sci. URSS 30 (1941) 301-305.

[24] P.R. Krishnaiah and M.M. Rao, Remarks on a multivariate gamma distribution. Amer. Math. Monthly 68 (1961) $342-346$.

[25] A.S. Krishnamoorthy and M. Parthasarathy, A multi-variate gamma-type distribution. Ann. Math. Statist. 22 (1951) 549-557.

[26] W.E. Leland, M.S. Taqqu, W. Willinger and D.W. Wilson, On the self-similar nature of Ethernet traffic (extended version). IEEE/ACM Trans. Networking 2 (1994) 1-15.

[27] S.B. Lowen and M.C. Teich, Fractal renewal processes generate 1/f noise. Phys. Rev. E 47 (1993) 992-1001.

[28] B.B. Mandelbrot, Long-run linearity, locally Gaussian processes, h-spectra and infinite variances. Technometrics 10 (1969) 82-113.

[29] B.B. Mandelbrot and J.W. Van Ness, Fractional Brownian motions, fractional noises and applications. SIAM Rev. 10 (1968) 422-437.

[30] A.P. Prudnikov, Y.A. Britshkov and O.I. Maritshev, Integrali i ryadi. Nauka, Moscow. Appl. Math. (1981), in Russian.

[31] H.R. Riedi, S.M. Crouse, J.V. Ribeiro and G.R. Baraniuk, A multifractal wavelet model with application to network traffic. IEEE Trans. Inform. Theory 45 (1999) 992-1018.

[32] B. Ryu and S.B. Lowen, Point process models for self-similar network traffic, with applications. Comm. Statist. Stochastic Models 14 (1998) 735-761.

[33] G. Samorodnitsky and M.S. Taqqu, Linear models with long-range dependence and finite or infinite variance, edited by D. Brillinger, P. Caines, J. Geweke, E. Parzen, M. Rosenblatt and M.S. Taqqu, New Directions in Time Series Analysis, Part II. Springer-Verlag, New York, IMA Vol. Math. Appl. 46 (1992) 325-340.

[34] T. Shiga and S. Watanabe, Bessel diffusions as a one-parameter family of diffusion processes. Z. Wahrsch. Verw. Gebiete 27 (1973) 37-46.

[35] A.N. Shiryaev, Probability. Springer-Verlag, New York (1996).

[36] S.E. Shreve, Steven shreve's lectures on stochastic calculus and finance, Online: www.cs.cmu.edu/chal/shreve.html

[37] Y.G. Sinai, Self-similar probability distributions. Theor. Probab. Appl. 21 (1976) 64-84.

[38] G. Szegö, Orthogonal polynomials, in Colloquium Publ., Vol. XXIII. American Math. Soc., New York (1936).

[39] M.S. Taqqu, V. Teverovsky and W. Willinger, Is network traffic self-similar or multifractal? Fractals 5 (1997) 63-73.

[40] G. Terdik, Bilinear Stochastic Models and Related Problems of Nonlinear Time Series Analysis; A Frequency Domain Approach. Springer-Verlag, New York, Lecture Notes in Statist. 142 (1999).

[41] G. Terdik, Z. Gál, S. Molnár and E. Iglói, Bispectral analysis of traffic in high speed networks. Comput. Math. Appl. 43 (2002) 1575-1583.

[42] W. Willinger, M.S. Taqqu, R. Sherman and D.V. Wilson, Self-similarity through high variability: Statistical analysis of Ethernet LAN traffic at the source level. Comput. Commun. Rev. 25 (1995) 100-113.

[43] T. Yamada and S. Watanabe, On the uniqueness of solutions of stochastic differential equations. J. Math. Kyoto Univ. 11 (1971) 155-167. 